Florida International University FIU Digital Commons

$11-10-2010$

\title{
Monitoring the Health of Plates with Simultaneous Application of Lamb Waves and Surface Response to Excitation Approaches
}

Gurjiwan Singh

Florida International University, gsing004@fiu.edu

DOI: $10.25148 /$ etd.FI10121003

Follow this and additional works at: https://digitalcommons.fiu.edu/etd

\section{Recommended Citation}

Singh, Gurjiwan, "Monitoring the Health of Plates with Simultaneous Application of Lamb Waves and Surface Response to Excitation Approaches" (2010). FIU Electronic Theses and Dissertations. 292.

https://digitalcommons.fiu.edu/etd/292 


\section{FLORIDA INTERNATIONAL UNIVERSITY}

Miami, Florida

\section{MONITORING THE HEALTH OF PLATES WITH SIMULTANEOUS APPLICATION OF LAMB WAVES AND SURFACE RESPONSE TO EXCITATION APPROACHES}

A thesis submitted in partial fulfillment of the requirements for the degree of MASTER OF SCIENCE in MECHANICAL ENGINEERING by

Gurjiwan Singh 
To: Dean Amir Mirmiran

College of Engineering and Computing

This thesis, written by Gurjiwan Singh, and entitled Monitoring the Health of Plates with Simultaneous Application of Lamb Waves and Surface Response to Excitation Approaches, having been approved in respect to style and intellectual content, is referred to you for judgment.

We have read this thesis and recommend that it be approved.

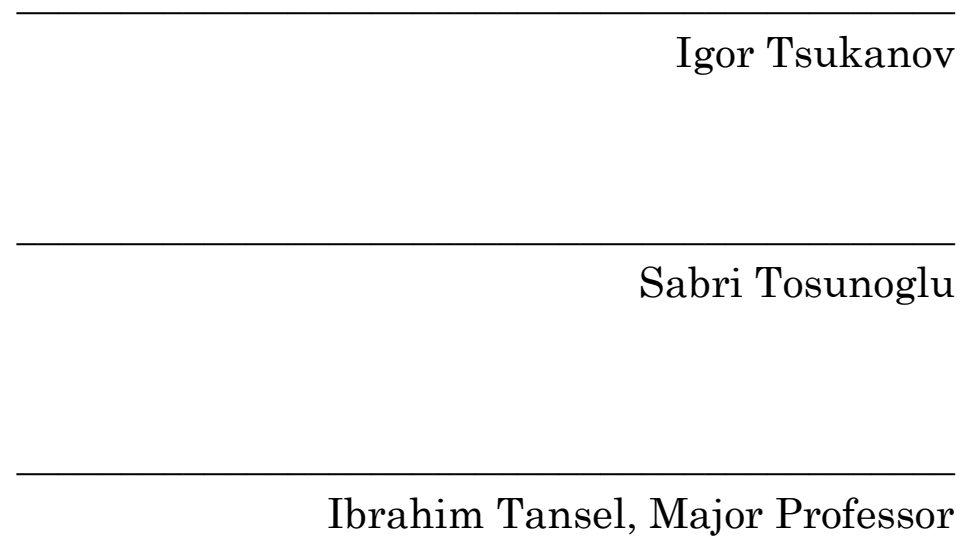

Date of Defense: November 10, 2010

The thesis of Gurjiwan Singh is approved.

Dean Amir Mirmiran

College of Engineering and Computing

Interim Dean Kevin O'Shea

University Graduate School

Florida International University, 2010 


\section{ACKNOWLEDGMENTS}

First and foremost I want to thank my mentor Dr. Ibrahim Tansel for his intellectual support and continual encouragement. His infectious enthusiasm and unlimited zeal have been major driving forces throughout my graduate carrier at Florida International University. I would also like to thank my committee members Dr. Sabri Tosunoglu and Dr. Igor Tsukanov for their guidance over the years.

Thanks also go out to my research mates Gurjashan Singh, Srikanth Korla, Dr. Mustafa Demetgul and Dr. Ming Li for their supporting and intellectual comments during this research. I also extend my gratitude to my friends Puneet K. Singh Gill, Amardeep Mann, Smith Pandya, Srikanth Korla for all the support they have lent me.

I am forever grateful to my parents who showed me the truth worth of hard work. Their unwavering faith and confidence in my abilities and in me is what has shaped me to be the person I am today. I would also like to thank my sister and my brother for their love, affection and support they have provided me in every step of my life. Finally, I would like to take opportunity to thank all my teachers at Florida International University. 


\section{ABSTRACT FOR THE THESIS \\ MONITORING THE HEALTH OF PLATES WITH SIMULTANEOUS \\ APPLICATION OF LAMB WAVES AND SURFACE RESPONSE TO \\ EXCITATION APPROACHES}

by

Gurjiwan Singh

Florida International University, 2010

Miami, Florida

Professor Ibrahim Tansel, Major Professor

Structural Health Monitoring (SHM) is a process of implementing a damage identification procedure for mechanical, aerospace and civil engineering infrastructure. Any change in the geometric properties, boundary conditions and behavior of material is defined as damage of these systems. In the past 10 years, there has been an accelerated increase in the amount of research related to SHM [1]. Hence, the increased interest in SHM to a wide range of industries and its correlated capability for significant life-safety and economic benefits has motivated the need for this thesis topic. The objective of this thesis study was to explore SHM approach to monitor and detect a change and/or damage in plates using Lamb wave propagation and surface response to excitation. First, the endurance of sensors and the adhesive used was evaluated. Next, the experimental data from the prepared samples was collected, compared, and evaluated. The obtained results indicated the severity and location of the defects. 


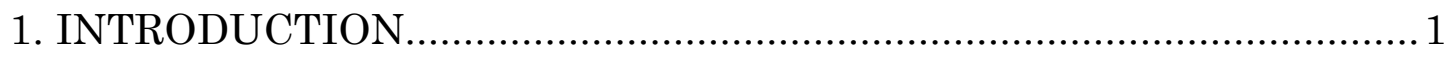

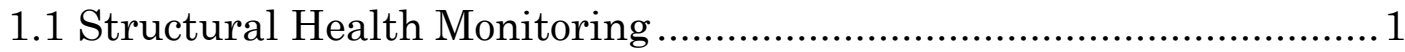

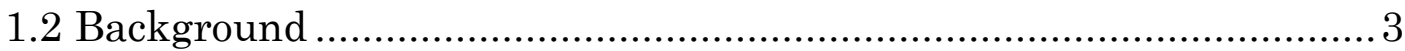

1.3 Lamb Wave Propagation for SHM ................................................ 5

1.4 Piezoelectric Wafer Active Sensors (PWAS) ........................................ 7

1.5 Guided waves (Lamb Wave) in plate like structures ........................... 8

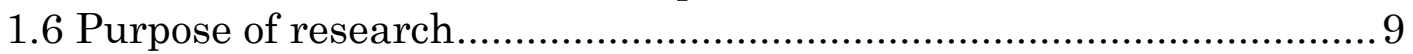

2. STRUCTURAL HEALTH MONITORING TECHNIQUES ....................11

2.1 Electromechanical Impedance (EMI) technique.................................. 11

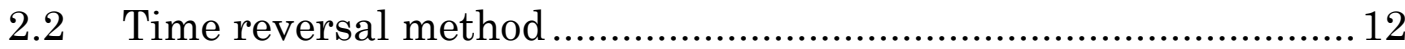

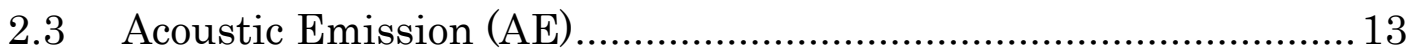

\section{DURABILITY AND SURVIVABILITY OF PWAS AND BONDING}

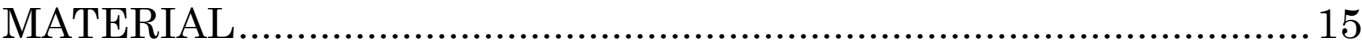

3.1 Testing the sample at room condition without exciting the piezoelectric transducers ............................................................. 15

3.2 Testing variation of the sweep sine wave response with time at room conditions ................................................................ 17

3.3 Testing samples at elevated temperatures inside a Furnace ........18

3.3.1 Performance of the PWAS and bonding material at $50^{\circ} \mathrm{C}$

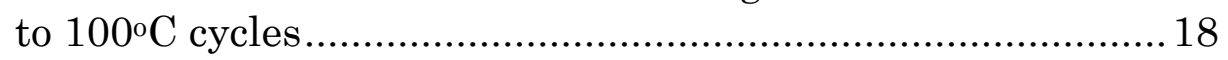

3.3.2 Performance of the Vishay M-Bond AE-10 adhesive at

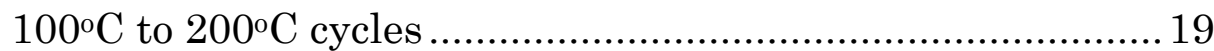

3.4 Testing piezoelectric elements and the bonding material

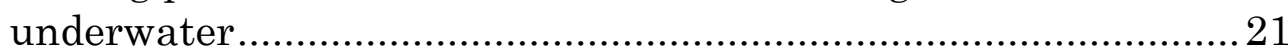

3.4.1 Testing durability of M-Bond 200 adhesive for underwater

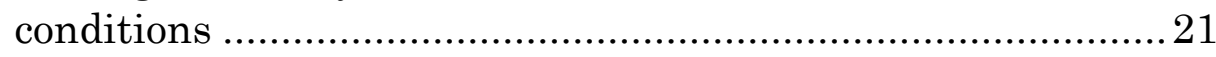

3.4.2 Testing durability of M-Bond AE-10 adhesive for

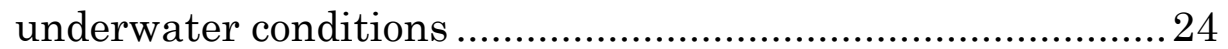

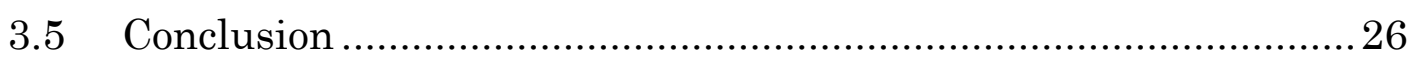

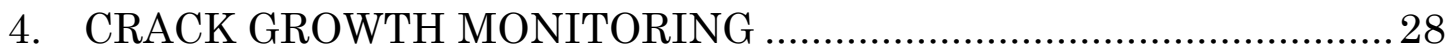

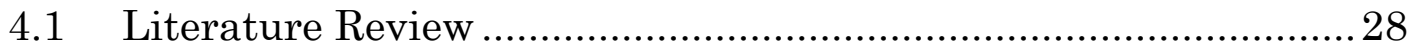

4.2 Methodology and experimental setup ………................................ 30

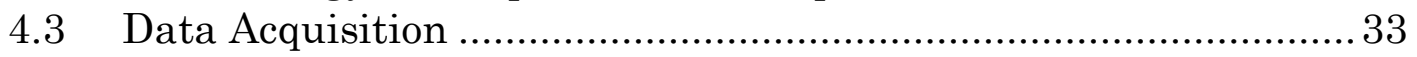

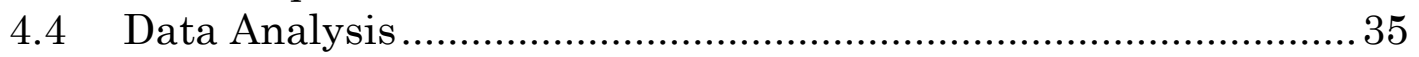

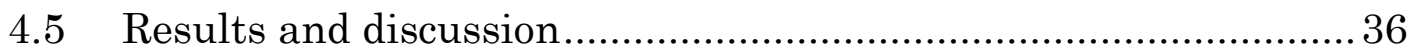

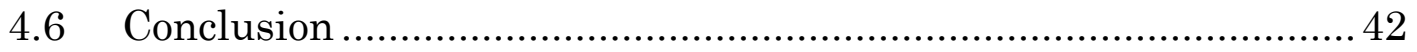

5 MONITORING THE DEFECTS IN WELDED PLATE JOINT ...........43

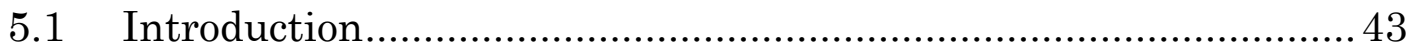

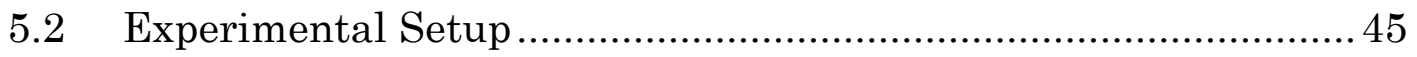


5.3 Methodology 47

5.4 Results and discussions: Lamb wave approach ............................. 47

5.5 Results and discussions: Surface Response to Excitation..............57

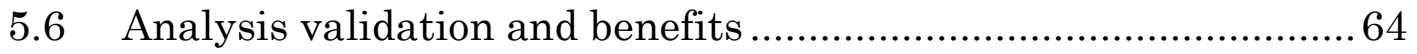

5.7 Conclusion .............................................................................. 70

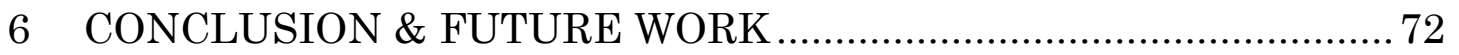

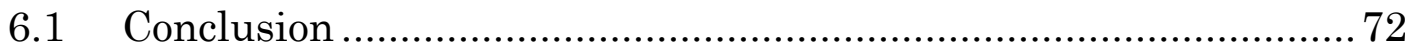

6.2 Recommendations for Future Work ............................................... 73

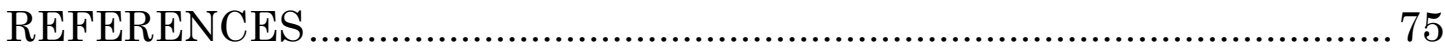


FIGURE

\section{LIST OF FIGURES}

Figure 1.1 Main components of advanced SHM-System ..............................2

Figure1.2 Benefits of SHM [CHA 02] ....................................................

Figure 1.3 Symmetric and Ant-symmetric Lamb wave modes................... 7

Figure 3.1 Small test plate with bonded piezoelectric sensors ................... 16

Figure 3.2 Frequency response variations with time................................. 16

Figure 3.3 Test plate showing sensor position........................................... 17

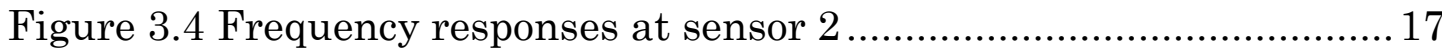

Figure 3.5 Testing sample in a furnace ................................................... 19

Figure 3.6 Frequency responses at the Sensor (3).....................................19

Figure 3.7 Test plate with one Metis MD1 (analog PZT/flex assemblies with 1/4"piezo) and two APC International D-018 ......20

Figure 3.8 Frequency response of (a) Metis MD1 piezo (b) APCI D-

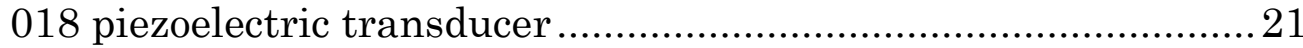

Figure 3.9.The Small and Big Test Plate with schematic diagram ...........22

Figure3.10 The Frequency Response of the Piezo 3 of the Small Plate.....23

Figure 3.11 The Frequency Response of the Piezo 3 of the Big Plate........23

Figure 3.12 Identical plate samples for underwater experiment ..............24

Figure 3.13 Frequency Response of the APC D-018 piezo of the Plate 1

Figure 3.14 The Frequency Response of the Metis MD1 of the Plate 2.....25

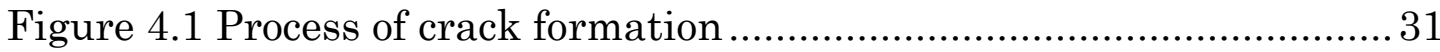

Figure 4.2 Aluminum plate with initial crack.......................................... 32

Figure 4.3.The three stages of the crack on the aluminum plate............... 32

Figure 4.4.Outline of the experimental data collection (Lamb wave approach) 
Figure4.5 Outline of the experimental data collection (Frequency response to excitation approach)

Figure 4.6 Analysis of the Lamb wave data by using the Stransformation

Figure 4.7.The voltage variation at the sensing piezoelectric element after the Lamb wave was generated.

Figure 4.8.The S-transformation at the interested frequency range. 38

Figure 4.9 Comparison of the envelopes of the signals when the crack had three different lengths. The crack length was 1", 1.5", and 2" in the Data 1, 2, and 3 respectively. 38

Figure 4.10.The measured frequency response characteristics at different crack lengths

Figure 4.11.Square of difference of the magnitude of signals 40

Figure 4.12.The sum of square of the difference between the first and second; and first and third frequency response characteristics.....41

Figure 5.1 Plate specimen showing actuator \& sensors location................4 45

Figure 5.2.Weld plate specimen with two cut lengths (1/4 \&1/8 inch) ...... 46

Figure 5.3 Welded plate sample (a) Drilling hole operation; (b) Showing hole diameter; (c) Figure showing positions of holes on sample. 46

Figure 5.4 Lamb Wave response for three stages of cut at sensor 1 .........48

Figure 5.5 Lamb Wave response for three stages of cut at sensor 2 .........49

Figure5.6 S-transformation calculation at sensor 1 for; (a) perfect sample (b) small cut (c) enlarged cut

Figure 5.7 S-transformation calculations at sensor 2 for; (a) perfect sample (b) small cut (c) enlarged cut 51

Figure 5.8 Comparison of the envelopes of the signals for three stages of defects (perfect, small cut and enlarged cut) at sensor 1 
Figure 5.9 Comparison of envelopes of the signals for three stages of defects (perfect, small cut and enlarged cut) at sensor 2 .

Figure 5.10Lamb wave response for perfect condition and three hole positions at sensor 1 .

Figure5.11 Lamb wave response for perfect condition and three hole

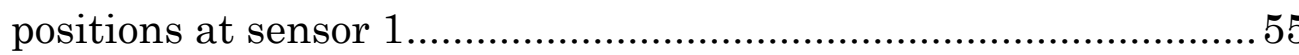

Figure5.12 S-transformation of dynamic response of perfect plate 56

Figure 5.13 Comparison of envelopes of signals for three identical hole positions

Figure 5.14 Sweep sine wave responses. 58

Figure 5.15 Sum of square of difference between defected and perfect sample.

Figure 5.16 Sum of square of difference at four different frequency intervals (a) Bar graph representation (b) Linear representation

Figure 5.17 Sweep sine wave response for three hole positions 62

Figure 5.18 Sum of square of difference between perfect and three identical holes signals

Figure 5.19 Comparison of sum of squares at different frequency intervals (a) Bar graph representation (b) Linear representation 64

Figure 5.20 Comparison of envelopes of two identical data sets. 65

Figure 5.21 Absolute differences between two identical sets of data in time domain (a) Difference between identical data 1 \& 2 (b) Difference between identical data $3 \& 4$ 67

Figure 5.22 Wavelet transformations of random data (a) First level (b) Second level (c) Fourth level 69 


\section{INTRODUCTION}

\subsection{Structural Health Monitoring}

In recent years, structural health monitoring (SHM) technology is increasingly being evaluated by the industry as a possible method for improving the safety and reliability of structures and to thereby reduce their operational cost [2]. SHM system consists of networks of embedded sensors that are permanently inserted into the structure and monitored over time. All in service structures, such as bridges, dams, spacecraft's, nuclear reactors, aircrafts and many other civil structures are undoubtedly benefited from the development of SHM system. Based on the sensor functionality, all SHM systems can be broadly classified into two types: (a) passive sensing and (b) active sensing. Passive SHM speculate the state of the structure using passive sensors that are monitored over time and fed back into the structural model to measure the structural response during an impact [47]. Passive SHM does not interact with the structure. The most significant passive sensing schemes are acoustic

emission (AE), external loads and stress/strain monitoring [3-4]. Whereas active SHM uses sensors to interrogate the structure in order to detect damage location, extent and intensity. Active sensors interact directly with the structure and find its state of health and reliability [5].In particular two kinds of active sensing methods; the impedance-based method and the lamb wave-based method have been principally utilized in SHM fields over the last decade [6-9]. However, passive diagnosis is volatile to ambient noise and typically has low sensitivity as damage may 
not be significant enough to influence the global response of the entire structure. In contrast, active diagnosis locates damage by exciting wellcontrolled transient waves to propagate through structures and then examining the responses [48].

To ensure the structural integrity of the structure using SHM-systems, four functions, shown in the figure 1.1 are essential: Event and fatigue monitoring, including remaining life assessment with interface to logistical support and damage monitoring [24].

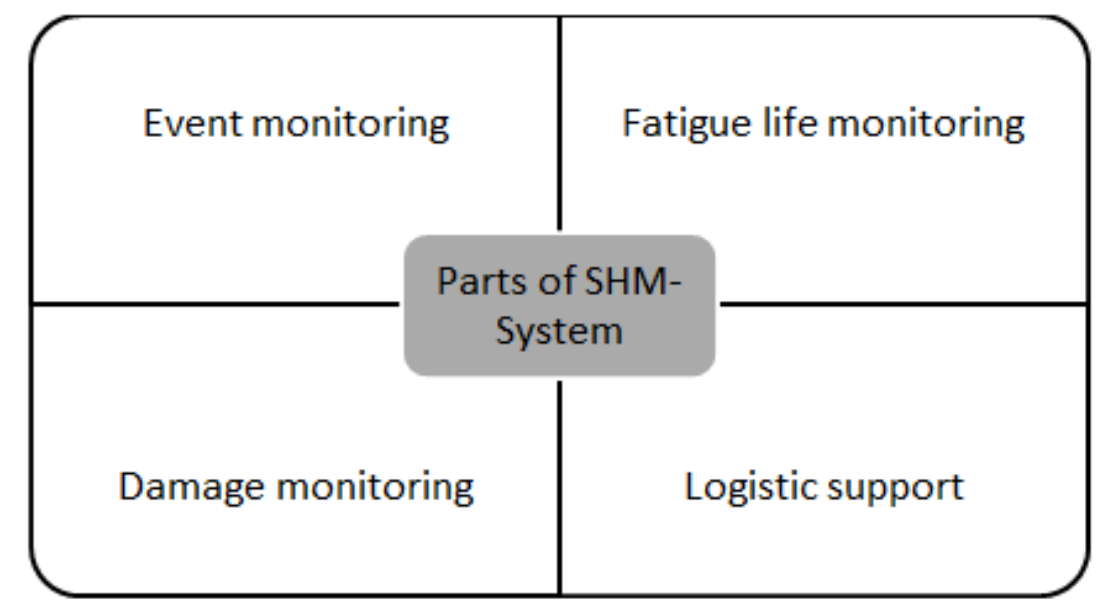

Figure 1.1 Main components of advanced SHM-System

The benefit of advanced SHM-System is based on an optimized use of the improvements of the following items:

a) Flexible Monitoring Locations

b) Reduced Scatter Factor

c) Component Monitoring 
d) Interface to Logistic Support

\subsection{Background}

Structural health monitoring (SHM) is a developing field with multiple applications. SHM sets out to determine the health of a structure by reading an array of sensors that are embedded into the structure and monitored over time. It assets the state of structural health through pertinent data processing and analysis, and may anticipate the remaining life of the structure. A good example of SHM application is the aircraft industry which, as one of the most innovative industries, has found that the application of SHM technology can contribute significantly to improve the flight safety, authenticity and readiness while reduce the maintenance cost [10]. The SHM initiated from early 1990s in many different parts of the world. It is difficult to identify the first form of SHM, but discounting simple periodic visual observation, formal structural monitoring and interpretation using recording instruments began in the latter half of the century and accelerated with the use of electronic data storage and computer data acquisition [1].The improvement of safety seems to be a strong motivation, in particular after some spectacular accidents due to unacceptable maintenance, for instance in aeronautical field, the accident of Aloha Airlines or, in the civil engineering field, the collapse of the Mianus River bridge, or due to ill controlled manufacturing processes, for example, the Injak bridge collapse. The problem of ageing structures was discovered in both fields, and subsequent programs were established. 


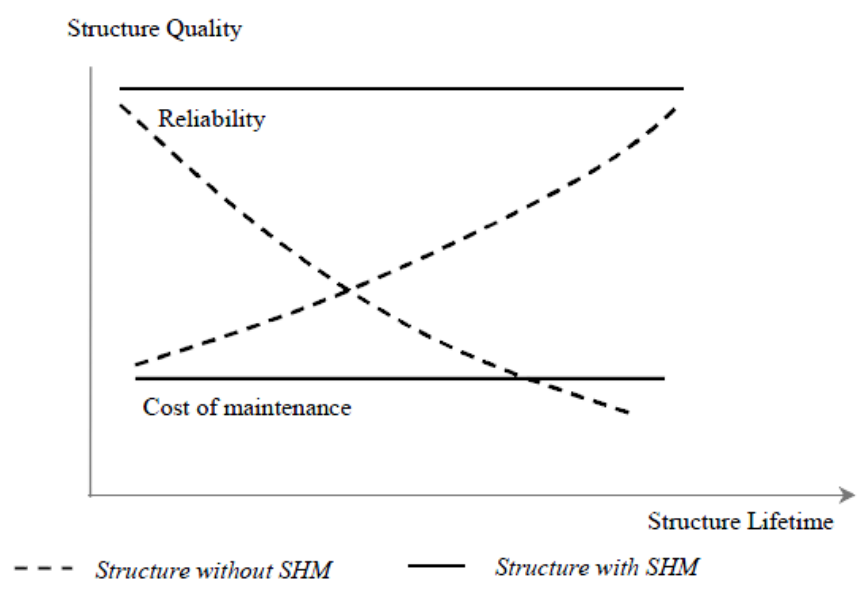

Figure1.2 Benefits of SHM

The economic motivation is stronger, principally for end-users. In effect, for structures with SHM system, the contemplate benefits are constant maintenance costs and reliability, instead of increasing maintenance costs and decreasing reliability for classical structures without SHM(Figure 1.2) Damage detection through guided wave NDE has gained extensive attraction in past few years. Guided waves, e.g. lamb waves in plates, are elastic perturbation that can propagate through long distances in thin wall structures with very little amplitude attenuation. In guided wave NDE, the number of sensors employed to monitor a structure can be significantly reduced. Being bulky and expensive, the size and the cost of the conventional NDE transducers is one of the major limitations in the path of development of guided wave NDE techniques to SHM methodology. The permanent installation of conventional NDE transducers onto a structure is not feasible, especially when weight and cost are at a premium such as in aerospace application. One popular way to improve SHM, damage detection, and NDE is to use piezoelectric wafer 
active sensors (PWAS) (Giurgiutiu, 2008). PWAS are small, lightweight and inexpensive and can be produced in various geometries. In SHM application, PWAS can be bonded onto the structural surface, or mounted inside buildup structures, or even embedded between the structural and non-structural layers of a composite construction.

Structural damage detection with PWAS is usually performed using the two widely used methods: (1) wave propagation (e.g., pitch- catch, pulse-echo, phased array), and (2) electromechanical (E/M) impedance. There has been a rising trend in the use of embedded piezoelectric wafer active sensors (PWAS) for the detection of material damage with Lamb wave techniques in recent years. Other methods using PWAS for SHM are still emerging. However, the modeling and characterization of Lamb wave generation, sensing for SHM, using these surface mounted or embedded PWAS still has a way to go. Also inadequately advanced are reliable damage detection algorithms that can assess the state of health of a structural with confidence and trust. The Lamb wave based damage detection techniques using PWAS is still in its innovative years. There are still many things to achieve in order to determine the state of structural health, i.e., structural integrity, damage presence (if any), damage size, and remaining life.

\subsection{Lamb Wave Propagation for SHM}

Discovered by Horace Lamb in 1917, Lamb waves can exist in platelike thin plates with parallel free boundaries. A comprehensive theory for 
such a wave was established by Mindlin in 1950 [12], in parallel with experimental work conducted by Schoch in 1952 and Frederick in 1962 [13]. The development of such a topic was driven essentially by its applications in medical industry during World War II. Subsequently in 1961, Worlton [14] introduced Lamb waves as a means of damage detection. All these master studies established the fundamentals of utilization of Lamb waves as a distinguished non-destructive evaluation (NDE) tool [11]. Lamb waves also known as guided plate waves, are a type of ultrasonic waves that remain guided between two parallel surfaces, such as the upper and lower surfaces of a plate or shell [5]. Lamb waves can travel over a long distance even in materials with a high attenuation ratio, such as carbon fiber-reinforced composites, and thus a broad area can be quickly examined. The entire thickness of the structure can also be interrogated by various Lamb modes, affording the possibility of detecting internal damage as well as that on surface. The potential damage types that a lamb wave based inspection can provide are summarized by Rose [15].Lamb waves are of two basic varieties, symmetric Lamb wave modes (S0, S1, S2...) and ant-symmetric Lamb wave modes (A0, A1, A2 ...) (Figure 1.3) [16]. 


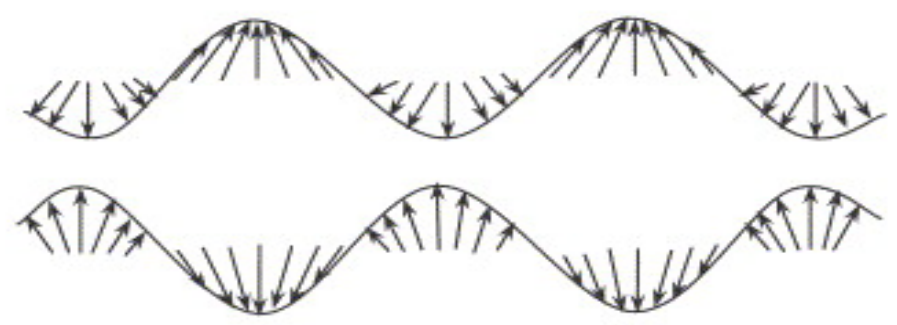

symmetric Lamb mode
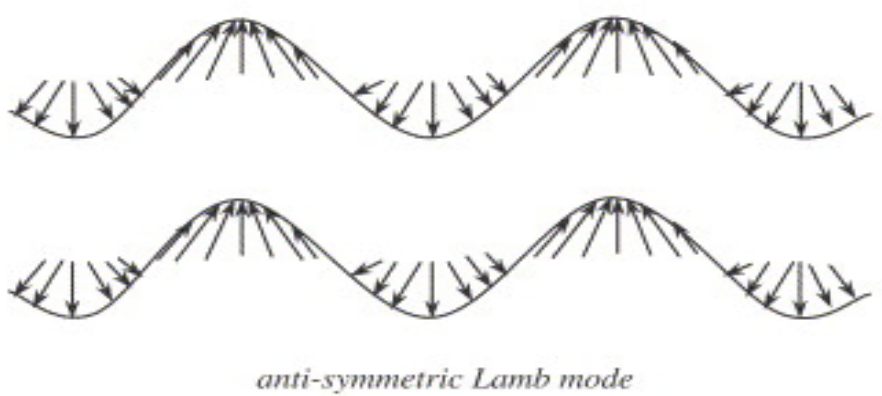

Figure 1.3 Symmetric and Ant-symmetric Lamb wave modes.

Both Lamb waves types are quite dispersive. At any given frequency (f), thickness (d) product (fd), a multitude of symmetric and antsymmetric Lamb wave may exist. Lamb waves can be generated using: conventional ultrasonic transducers [21,22], piezoelectric transducer [5,9,17-18] or laser source [19,20]. For its sophistication in damage detection for advanced composites, substantial efforts have been directed to Lamb wave study, especially in the past decade.

\subsection{Piezoelectric Wafer Active Sensors (PWAS)}

Piezoelectric wafer active sensors (PWAS) are small, lightweight and they are relatively low cost transducers. PWAS can be bonded to the structure or inserted into the layer of a composite structure. When permanently embedded/attached into the structure, PWAS provide the bidirectional energy transduction from the electronics into the structure, 
and from the structure back into the electronics. PWAS were initially used for vibration control and later adopted for damage detection. Initially used by Crawley and de Luis, Crawley and Anderson and others for structural vibration control. The use of PWAS in active SHM has been explored experimentally by Chang, Keilers and Chang, Lakshmanan and Pines, Moulin, Wang and Chang, Dupont, Monier, Kessler, Lin and Yuan, Diamanti, Ihn and Chang, Koh and others. Pitch-catch, pulse-echo, and phased array have been experimentally demonstrated. These successful experiments have positioned PWAS as an enabling technology for active SHM implementation [23].

PWAS operate on the piezoelectric principle that couples the electrical and mechanical variables in the material. When the applied stress on the sensor is converted into electric charge, it is known as the direct piezoelectric effect and when the strain is produced upon voltage applied on the sensor, it is known as the inverse effect. Piezoelectric transducer is the most popular, because it can be light and thin. Moreover, it can work as a strain sensor/exciter, resonator and can work both as generator and receiver of elastic waves.

\subsection{Guided waves (Lamb Wave) in plate like structures}

Lamb waves in plates take place in more than one mode of two kinds called symmetric and anti - symmetric modes. These waves are an elastic vibration that propagates in a solid plate with free boundaries, for which the displacements correspond to various basic propagation modes [67]. For 
a given plate thickness and frequency, there exists a finite number of such propagation modes specified by their phase velocities. A set of dispersion curves generally represents the complete description of such propagation characteristics for plates. Dispersion curves illustrate the plate mode phase velocity as a function of product of the frequency and thickness. Several kinds of materials through a lot of work are tested to calculate such dispersion curves. Viktorov [68] describes a method to obtain the description curves for isotropic homogeneous plate. Nayfeh and Chimenti [69] have computed dispersion curves for propagation of lamb waves in monoclinic media and multilayered plates.

\subsection{Purpose of research}

The main purpose this thesis is to monitor, analyze and detect various defects in plates/plate like structures using two different structural health monitoring (SHM) techniques. The newly developed piezoelectric materials with strong electromechanical coupling and potential for usage as sensors as well as actuators attracted convincing attention. Piezoelectric wafer active sensors being light and thin have the potential for in-situ testing through inexpensive and unobtrusive deployment. Experimental procedures were followed to evaluate the durability and sustainability of the piezoelectric transducers and bonding materials.

SHM techniques with highest probability of success were thoroughly analyzed and validated, including frequency response method and Lamb wave method. For each of these methods, an experimental 
procedure was followed to monitor and distinguish different defects existing in plates. In the end, recommendations are offered for structural health monitoring techniques based on the results presented in this thesis. 


\section{STRUCTURAL HEALTH MONITORING TECHNIQUES}

\subsection{Electromechanical Impedance (EMI) technique}

During the last ten years, the electromechanical impedance (EMI) technique has emerged as a universal cost-effective method for structural health monitoring (SHM) and nondestructive evaluation (NDE) of all types of structures and systems. In this technique a PZT patch, surface bonded to the monitored structure, employs ultrasonic vibrations at high frequency range, typically of the $\mathrm{kHz}$ range, to derive a characteristic electrical signature of the structure in frequency domain, containing vital information concerning the phenomenology nature of the structure. Electro-Mechanical admittance, which is the measured electrical parameter, can be decomposed and analyzed to extract the impedance parameter of the host structure [27-34]. Any change in the mass, stiffness,

or damping of the structure (due to damage) would result in a change in the admittance of the PZT [26]. In the EMI technique, the bonded PZT patch is electrically excited by applying an alternating voltage across its terminals using an impedance analyzer. This produces deformation in the patch as well as in the local area of the structure surrounding it. The response of the area is transferred back to the PZT in the form of the admittance, comprising of the conductance and the susceptance. Hence, the same PZT acts as an actuator as well as a sensor. Any damage to the structure manifests itself as a deviation in the admittance signature, which serves as an indication of the damage. In this manner, the PZT 
patch, acting as piezo-impedance transducer, enables structure identification, health monitoring and NDE [25, 35-37].

\subsection{Time reversal method}

The time reversal method was developed by Fink in connection with the pitch-catch NDE method. The signal sent by the transmitter arrives at the receiver after being modified by the medium in which it travels. If the received signal is time reversed and sent back from the receiver to the transmitter, the effect of the medium is also reversed. This reversal is quite spectacular in the case of dispersive lamb waves. In a series of remarkable experiments, Ing and Fink showed that the extensive dispersion of certain Lamb wave modes can almost completely compensated through the time reversal method. Deutsch also used to concept of reversing the reflected waves in order to focus a linear array of conventional ultrasonic transducers transmitting Rayleigh and Lamb waves [38-40]. The time reversal method has proven especially useful in ultrasonic imaging of difficult media [39].

In SHM, the time reversal method has been recently reported by several authors. Wang used the time reversal method on an aluminum plate [41]. Sohn and Park used the time reversal method to detect damage in composite panels [42]. Their approach uses the assumption that "because the time reversibility of waves is fundamentally based on the linear reciprocity of the system, this linear reciprocity and the time reversal break down if there exist any source of wave distortion due to 
wave scattering along the wave path. Therefore, by comparing the discrepancy between the original input signal and the reconstructed signal, damage could be detected." This approach seems to remove the need for a historical baseline, which is inherent in other SHM methods [23]. In addition, the method uses wavelet signal generation and processing to enhance the time reversal process in the case of multimode Lamb wave propagation.

\subsection{Acoustic Emission (AE)}

Acoustic emission (AE) is a non-destructive method that is sensitive to mechanical waves created in many materials by sudden displacement due to microscopic stress release. On the surface, AE signals are recorded by either surface-mounted piezo-electric transducers or without contact by optical interferometer. AE analysis is ideally suited for investigation of advanced fiber-reinforced polymer composites [45]. In acoustic emission (AE), a transient elastic wave is generated by the rapid release of energy from a localized source or within a material. The elastic energy propagates as a stress wave in the structure and is detected by one or more $\mathrm{AE}$ sensors. A stress wave may be generated by moving dislocations, crack growth and propagation, fiber breaks, disbonds, plastic deformation etc. AE differs from other NDT methods in two respects. First, the signal has its origin in the material itself and is not introduced from an external source. Second, AE detects the movement or strain, whereas most other

methods detect existing geometric discontinuities or breaks [44]. One of 
the main objectives of the $\mathrm{AE}$ technique is to discriminate among different sources of damage. The parameter analysis of an AE event evaluates and correlates AE features such as counts, amplitude, rise time, energy, etc. the classification of these parameters drives the investigator towards the correlation of the AE with its source [43]. In many cases, the size and shape of AE sensors limit continuous AE monitoring to stationary composite structures, such as, pressure vessels and pipelines [46]. 


\section{DURABILITY AND SURVIVABILITY OF PWAS AND BONDING MATERIAL}

Group of experiments have been performed to evaluate the effectiveness of two types of the bonding material at different conditions. Multiple piezoelectric elements (Metis MD1 analog PZT/flex assemblies with 1/4" piezo, APC international (dia. 0.50" and 0.25") piezo) were attached to 2024 tempered aluminum plates using two different bonding materials (M-Bond 200 and M-Bond AE-10)and were subjected to different conditions. One of the piezoelectric elements was excited with sweep sine wave and the response at other piezoelectric elements was recorded by real time analyzer SRT780 and then evaluated for the detection of any defect /damage in the piezoelectric element and bonds. Tested conditions include keeping the samples at room temperature conditions, elevated temperatures and under water conditions.

3.1Testing the sample at room condition without exciting the piezoelectric transducers

A test plate was prepared and four piezoelectric transducers were attached on it (Figure 3.1).M-bond 200 was used to bond the APC International (D-0.50) piezoelectric sensor. Excitation was applied to the piezoelectric sensor numbered (1) and the sweepsine wave response of other piezoelectric sensors was recorded, as presented in figure 3.2. 

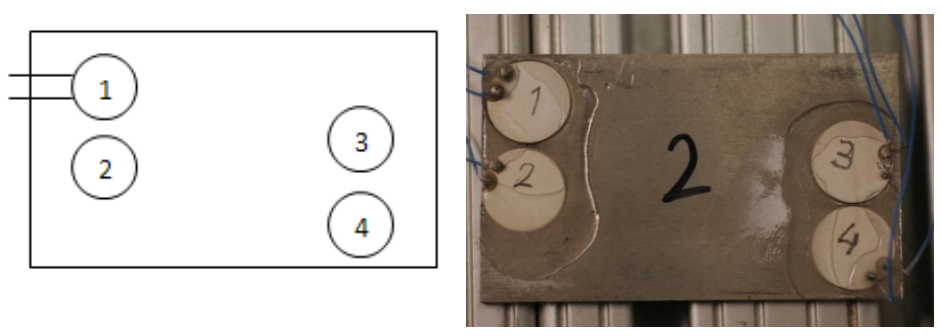

Figure 3.1 Small test plate with bonded piezoelectric sensors

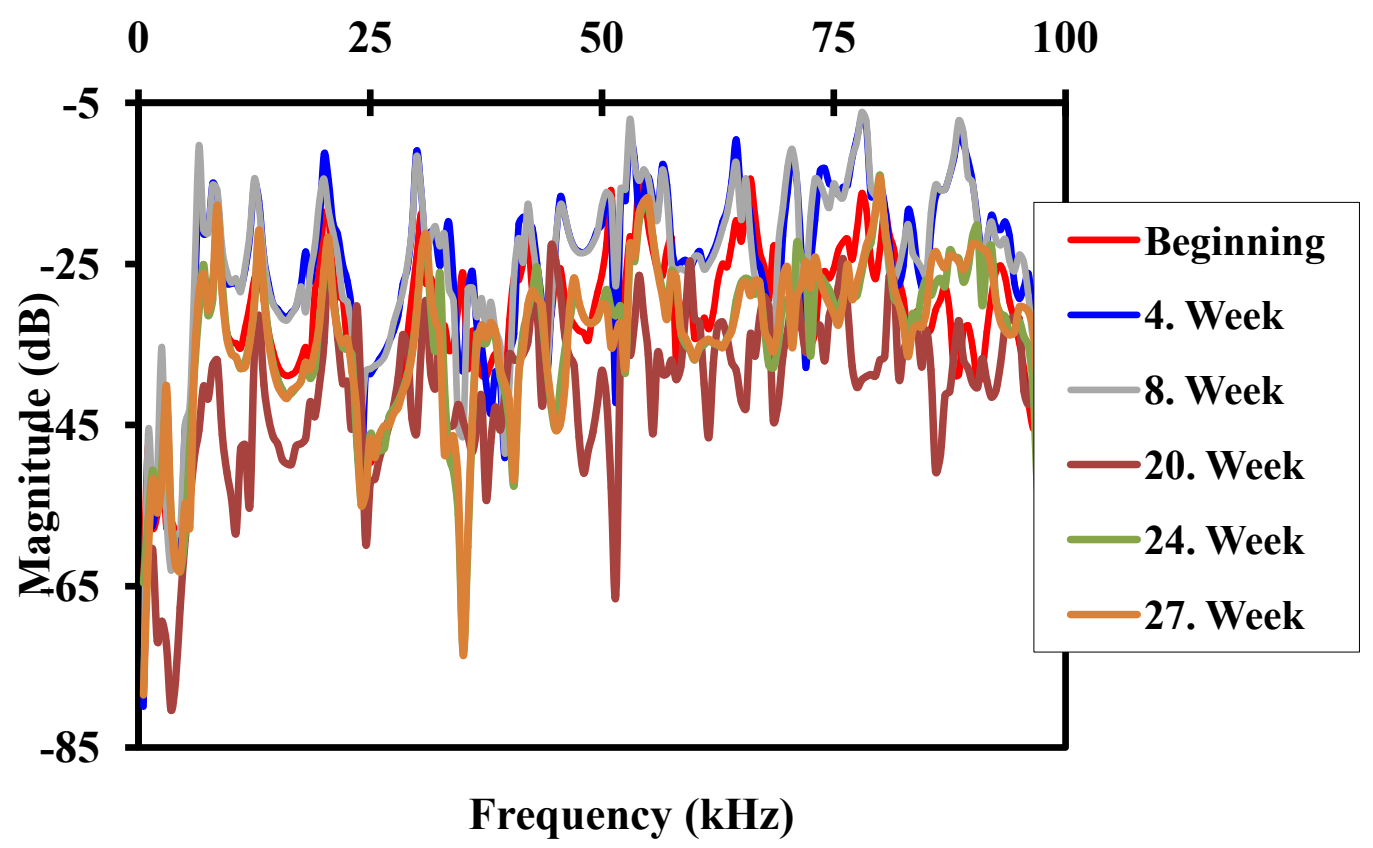

Figure 3.2 Frequency response variations with time

The result shows that the sweep sine wave transmission characteristics between two piezoelectric sensors slightly changed with time. This change indicates that even if the SHM system is going to operate at the ideal condition, it should be designed by considering that there would be some difference at the transmission characteristics. There was no deterioration of the signal within 27 week period. These results validates that the test setup worked effectively during the test period. 
3.2Testing variation of the sweep sine wave response with time at room conditions

A test plate with four piezoelectric sensors was prepared to evaluate the degradation of the bonding material and piezoelectric sensors over time, when the excitation was applied. M-Bond 200 was used to bond the APC International (D-0.50) piezoelectric sensor. Sensor numbered (1) was excited at $13 \mathrm{KHz}$ frequency continuously, and the sweep sine wave response at the other piezoelectric sensors was recorded (Figure 3.3).
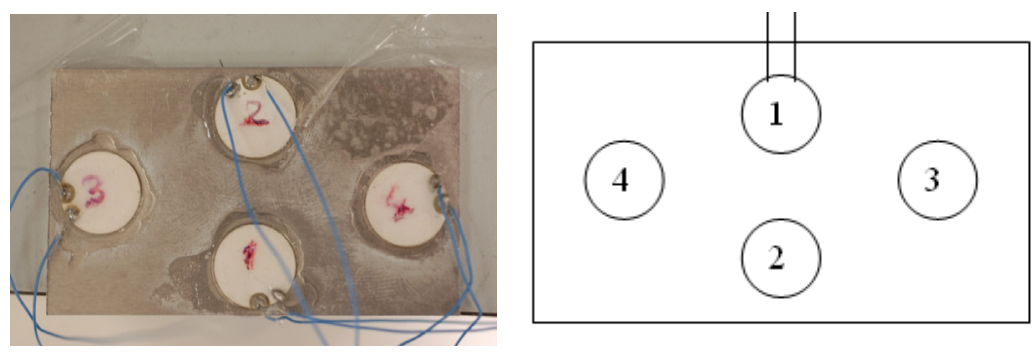

Figure 3.3 Test plate showing sensor position

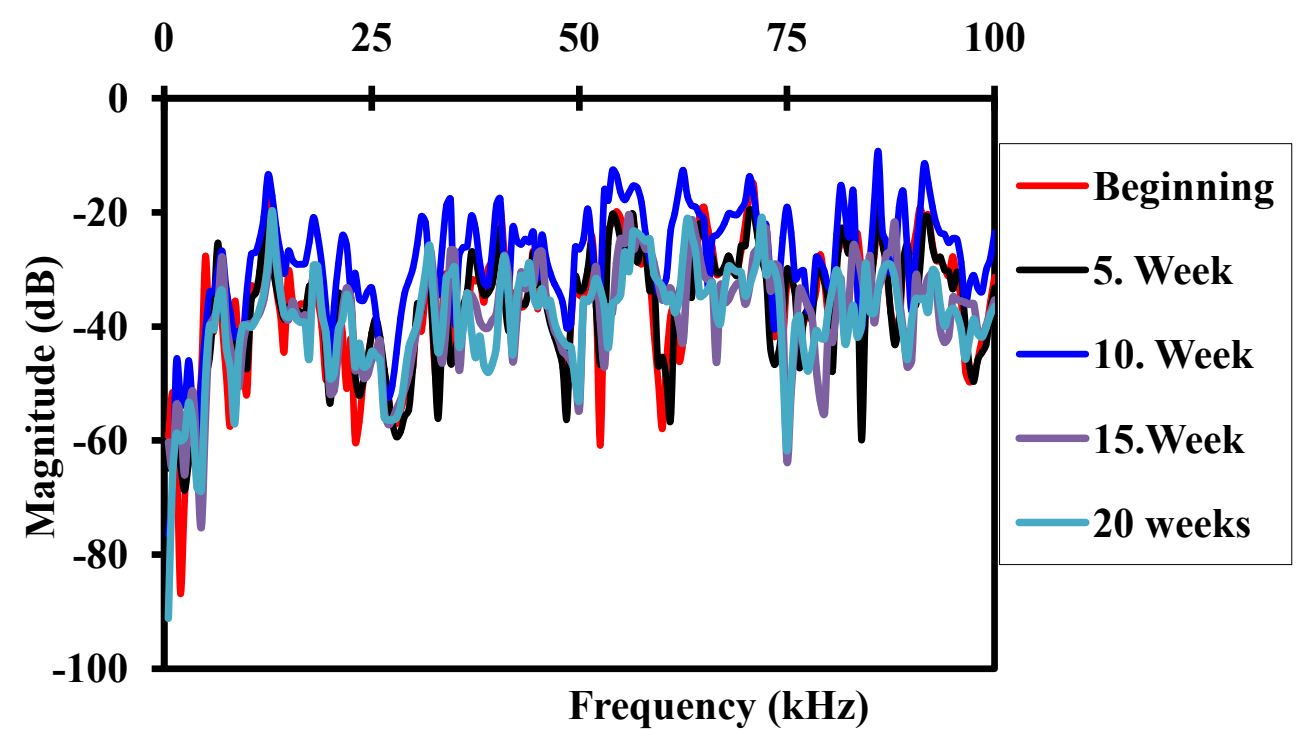

Figure 3.4 Frequency responses at sensor 2 
The sweep sine response at the piezoelectric sensor is presented in the figure 3.4. As seen, there is a small change at the frequency response characteristics of the transmission of the sweep sine wave between the piezoelectric transducers.

3.3Testing samples at elevated temperatures inside a Furnace

Three different sets of experiments were performed at the elevated temperatures by using two types of bonding material and piezoelectric elements.

3.3.1 Performance of the PWAS and bonding material at $50^{\circ} \mathrm{C}$ to $100^{\circ} \mathrm{C}$ cycles

A test plate with 4 piezoelectric elements was prepared. M-Bond 200 was used to bond the APC International (D-0.50) piezoelectric elements. The test plate was kept in the furnace and exposed to a temperature of $100^{\circ} \mathrm{C}$ for the first week and at $200^{\circ} \mathrm{C}$ for the second week (figure 3.5). In the second week it was observed that the glue gets very soft and started to pull off from the surface. After this observation, the temperature cycle was adjusted between $50^{\circ} \mathrm{C}-100^{\circ} \mathrm{C}$. The swept sine wave response was then obtained upon exciting piezoelectric element numbered 2 and recording the output at other piezoelectric element (figure 3.6). The characteristics of the frequency response changed every week, and in the $8^{\text {th }}$ week the signal characteristics were significantly different. Thus it can be said that 

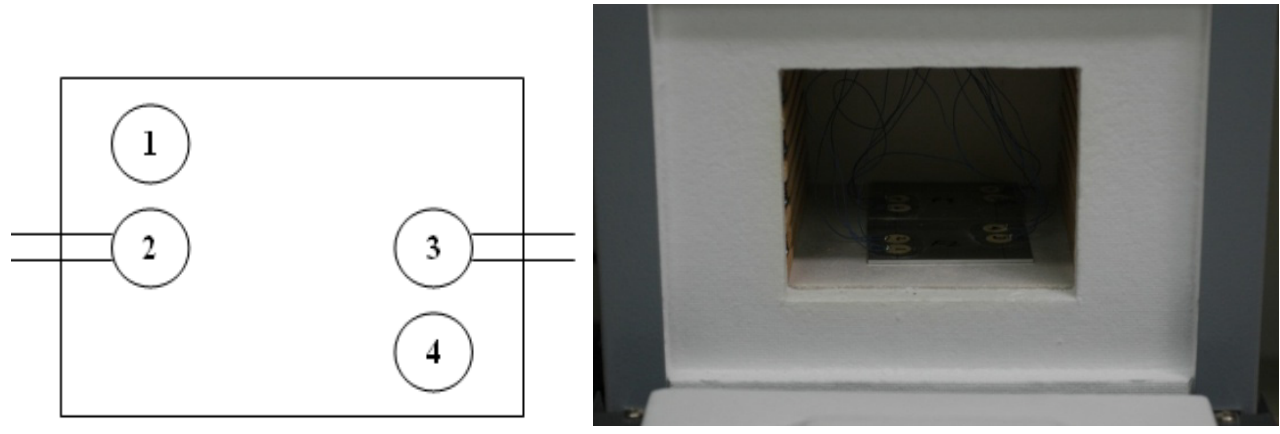

Figure 3.5 Testing sample in a furnace

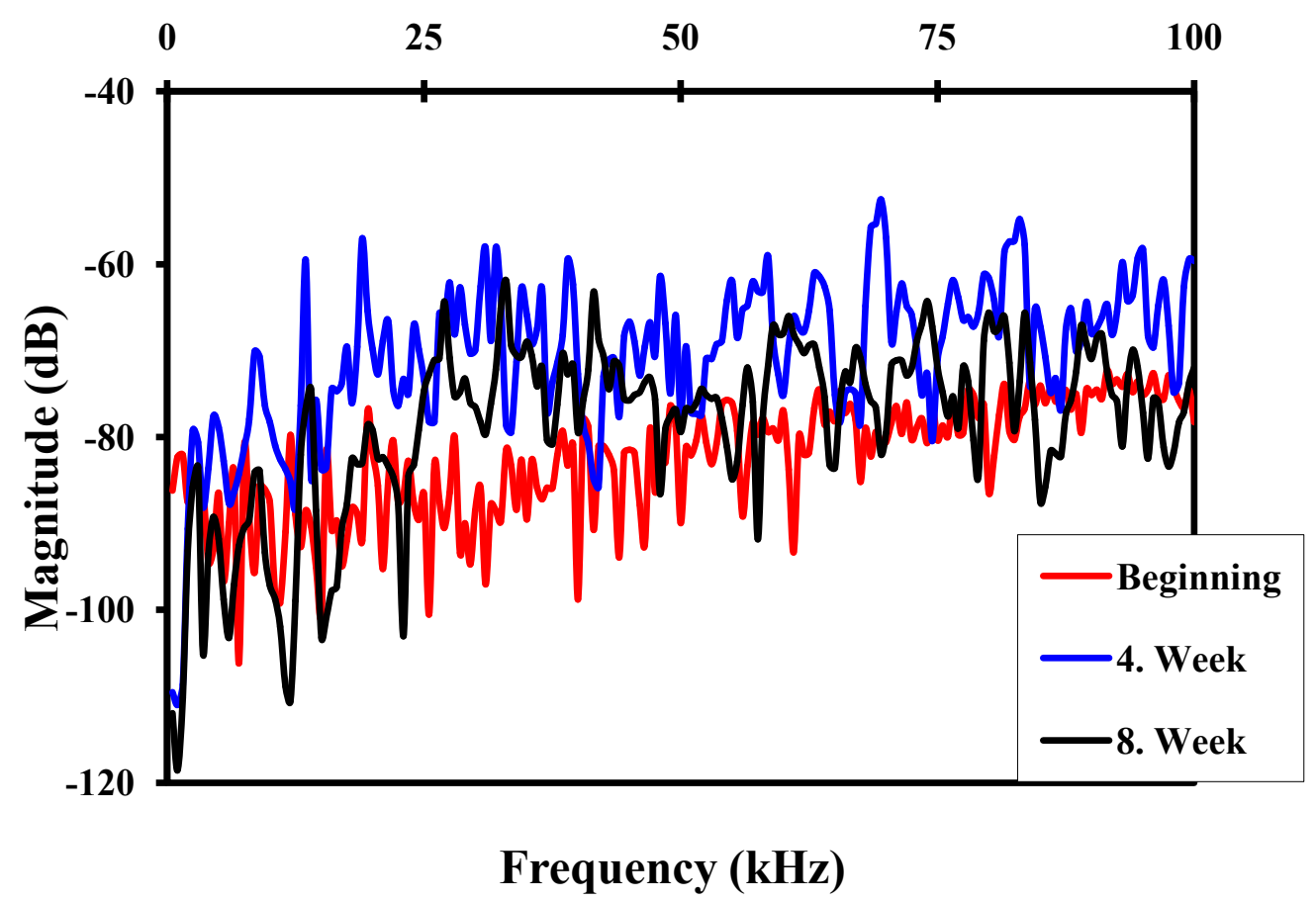

Figure 3.6 Frequency responses at the Sensor (3)

3.3.2 Performance of the Vishay M-Bond AE-10 adhesive at $100^{\circ} \mathrm{C}$ to $200^{\circ} \mathrm{C}$ cycles

A new test plate was prepared by attaching one Metis MD1 (analog PZT/flex assemblies with 1/4"piezo) and two APC International (D-.500) sensors to the aluminum plate by using the M-Bond AE-10 adhesive (figure 3.7). The test plate was kept in the furnace and exposed to a temperature variation of $200^{\circ} \mathrm{C}-100^{\circ} \mathrm{Cfor}$ alternate weeks. The frequency 
response plots for the two week long test period are presented in figure 3.8. Although the hardness of the bond looked fine, the frequency response of the transmission characteristics of the piezo couple changed very drastically after the second week when it operated at $200^{\circ} \mathrm{C}$. The data taken at the beginning of this experiment showed a settling in effect, i.e., some amplitude reduction during the first cycle, followed by a leveling off of the variation. Based on the observations, it is not recommended to use the piezoelectric elements and adhesive at $200^{\circ} \mathrm{C}$.

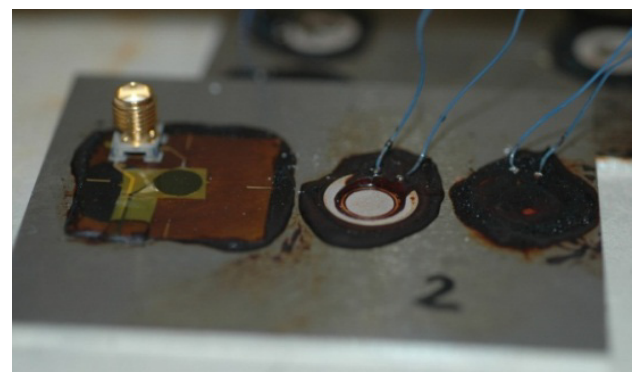

Figure 3.7 Test plate with one Metis MD1 (analog PZT/flex assemblies with 1/4"piezo) and two APC International D-018

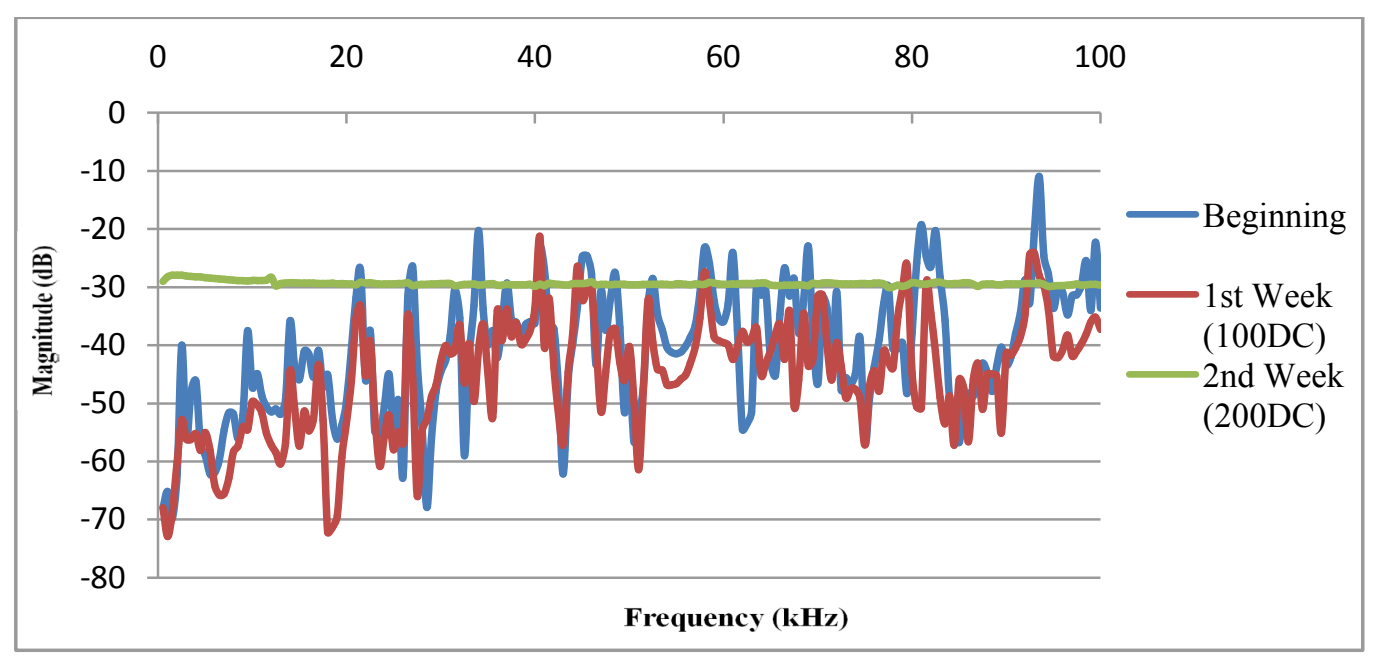

(a) 


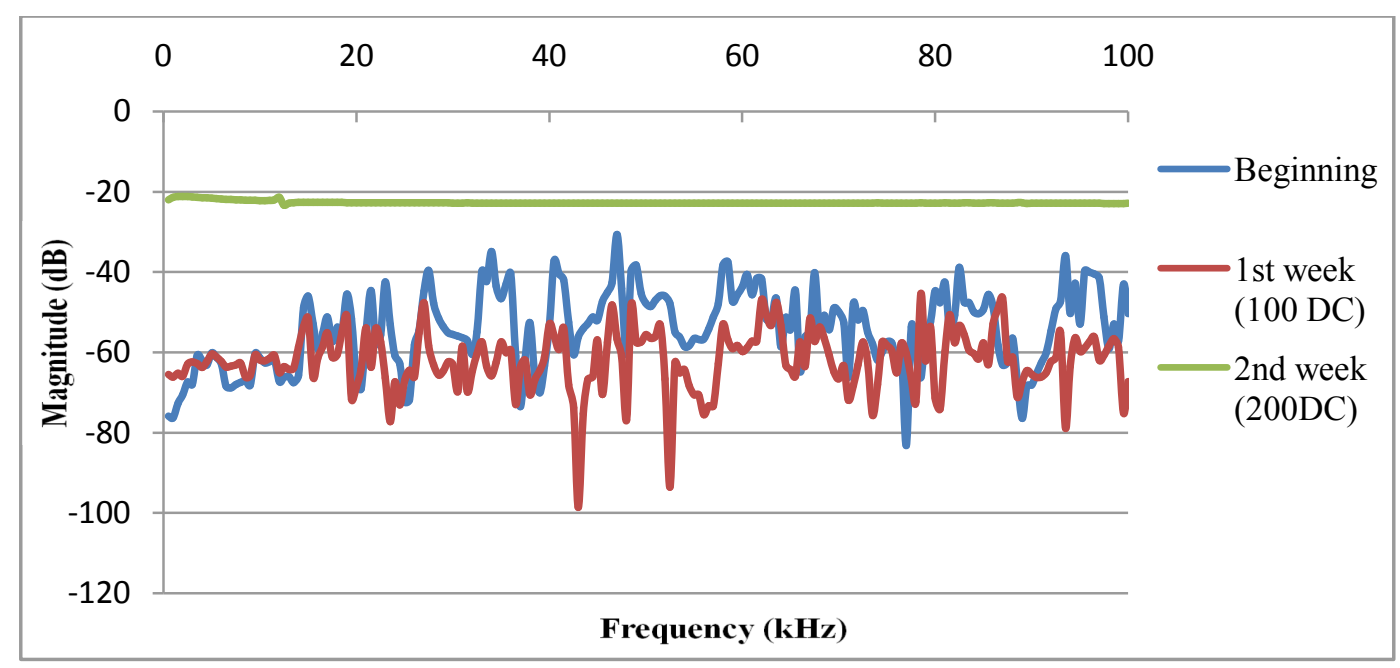

(b)

Figure 3.8 Frequency response of (a) Metis MD1 piezo (b) APCI D-018 piezoelectric transducer

3.4Testing piezoelectric elements and the bonding material underwater

Test plates were prepared by using two adhesives, to test the performance of the SHM system under water. Liquid tape was used in order to insulate the connections of piezoelectric sensors.

\subsubsection{Testing durability of M-Bond 200 adhesive for underwater} conditions

Two test plates with identical material properties and different geometric properties referred as small plate and big plate were prepared by bonding four APC International (D-0.50) piezoelectric element using MBond 200 adhesive. The location of piezoelectric sensors on the plate is presented in figure 3.9. The test specimens were submerged in water and the frequency response was recorded every four weeks. During the experiment, piezo numbered (2) was subjected to sweep sine wave and 
frequency response of piezo numbered 3 was recorded. The frequency response characteristics of the plates significantly changed every week as presented in figure 3.10 and figure 3.11 .

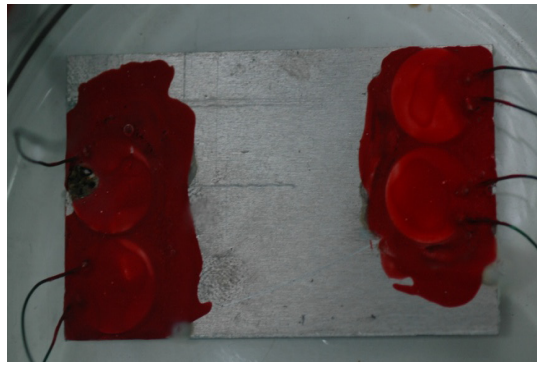

The small Test Plate with 4

Piezoelectric Elements

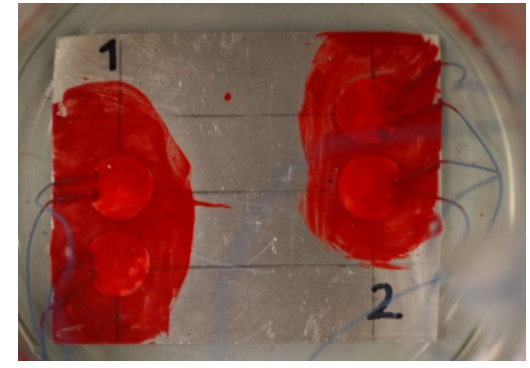

The Big Test Plate with 4

Piezoelectric Elements

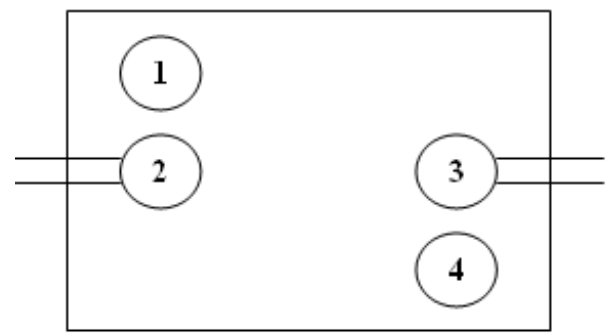

Figure 3.9.The Small and Big Test Plate with schematic diagram 


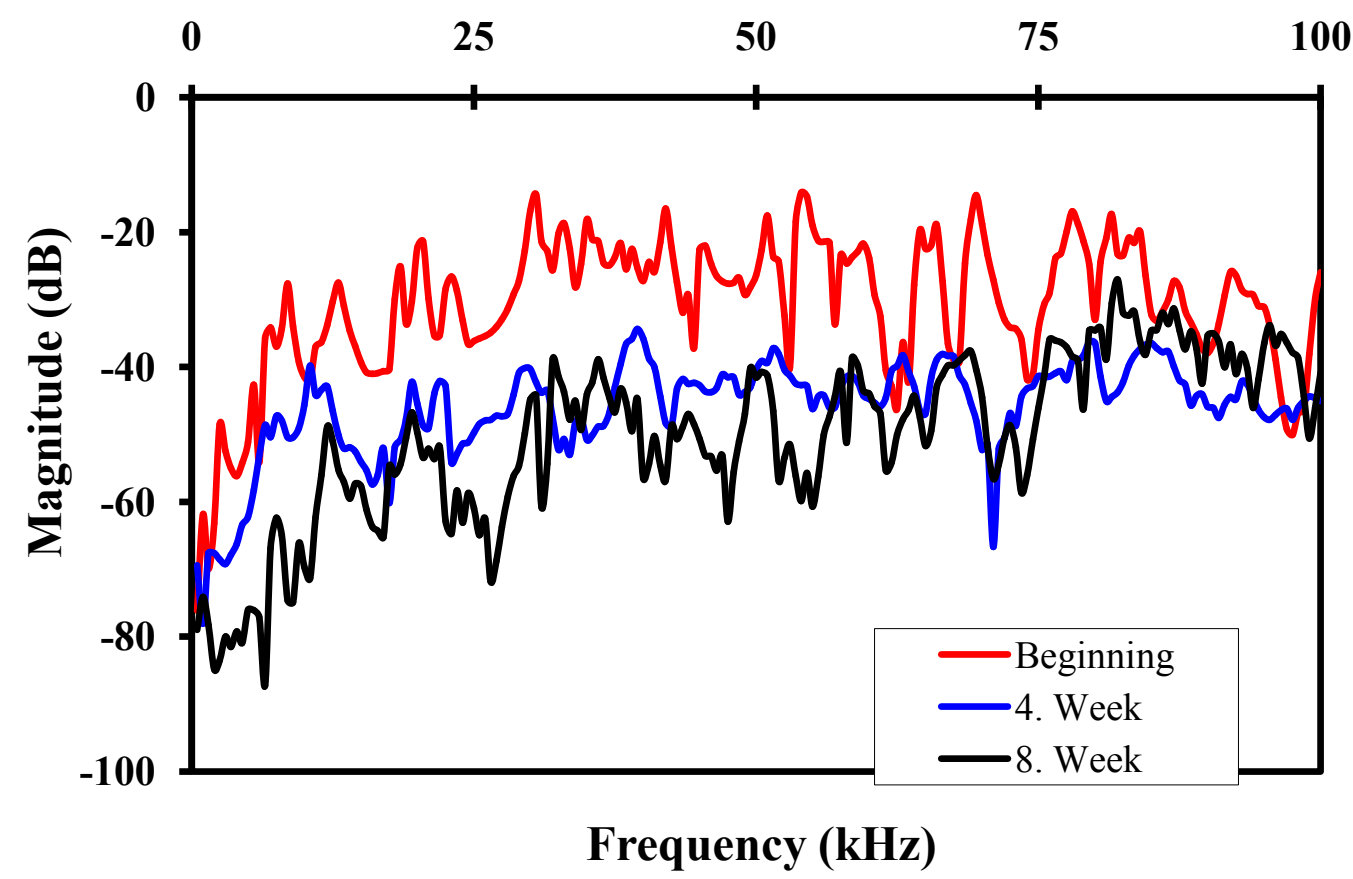

Figure3.10 The Frequency Response of the Piezo 3 of the Small Plate

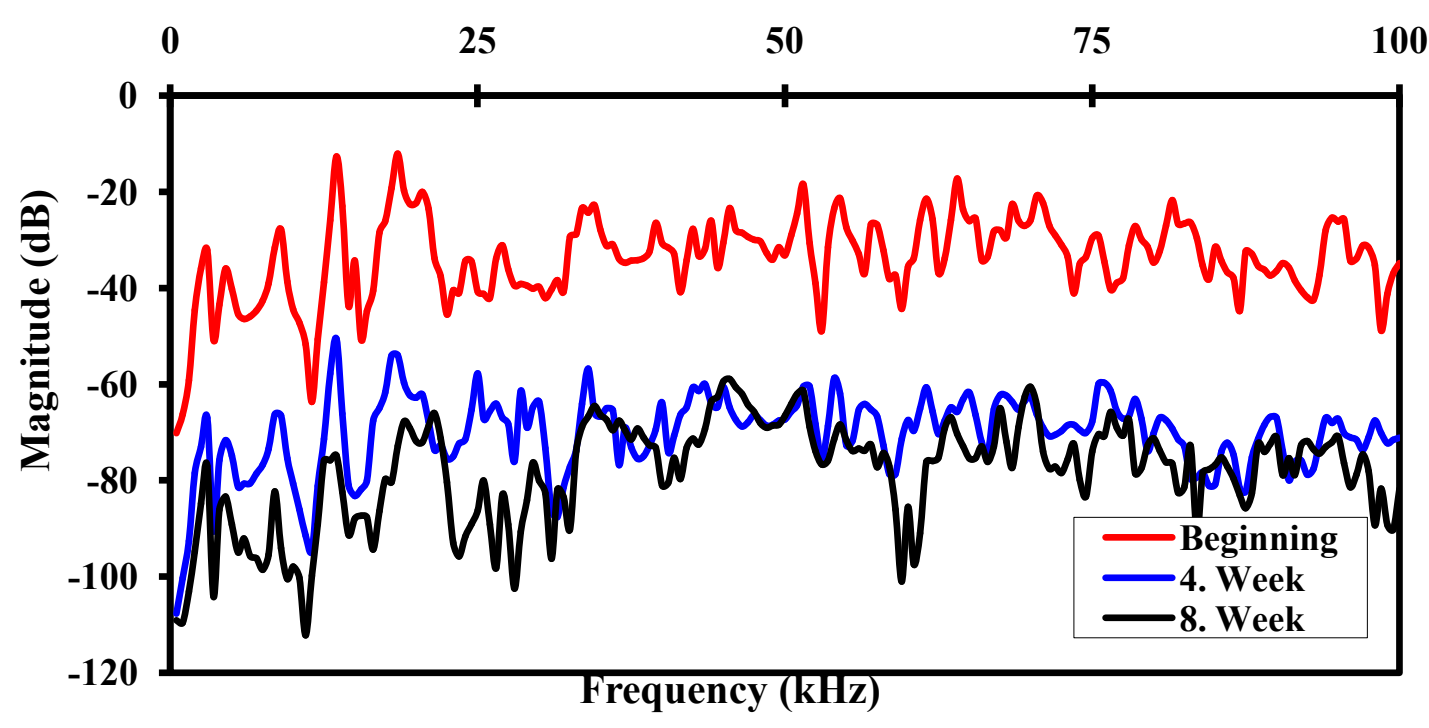

Figure 3.11 The Frequency Response of the Piezo 3 of the Big Plate

It can be clearly seen from the above plots that there was a settling in effect after the first cycle, and then there was no change/very little 
amplitude reduction. But there was no sign of physical deterioration of the bonding material (M-Bond 100).

3.4.2 Testing durability of M-Bond AE-10 adhesive for underwater conditions

Two test plates referred to as plate 1 and plate 2 (figure 3.12) with identical geometrical and material properties were prepared by bonding three piezoelectric elements. That includes, One Metis MD1 (analog PZT/flex assemblies with 1/4"piezo) sensor and two APC International (D0.50) sensors attached to aluminum plate by using the M-Bond AE-10 adhesive. The frequency responses of piezoelectric elements were plotted in figure 3.13 and figure 3.14 while one of the elements was subjected to sweep sine wave.

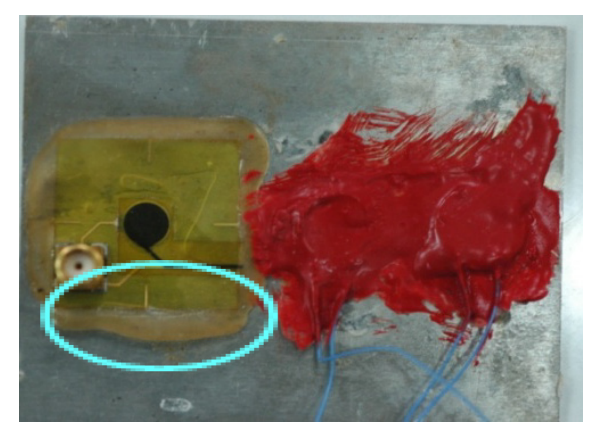

Sample Plate 1

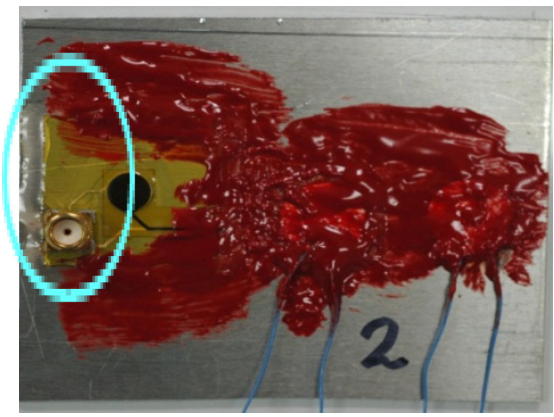

Sample Pate 2

Figure 3.12 Identical plate samples for underwater experiment 


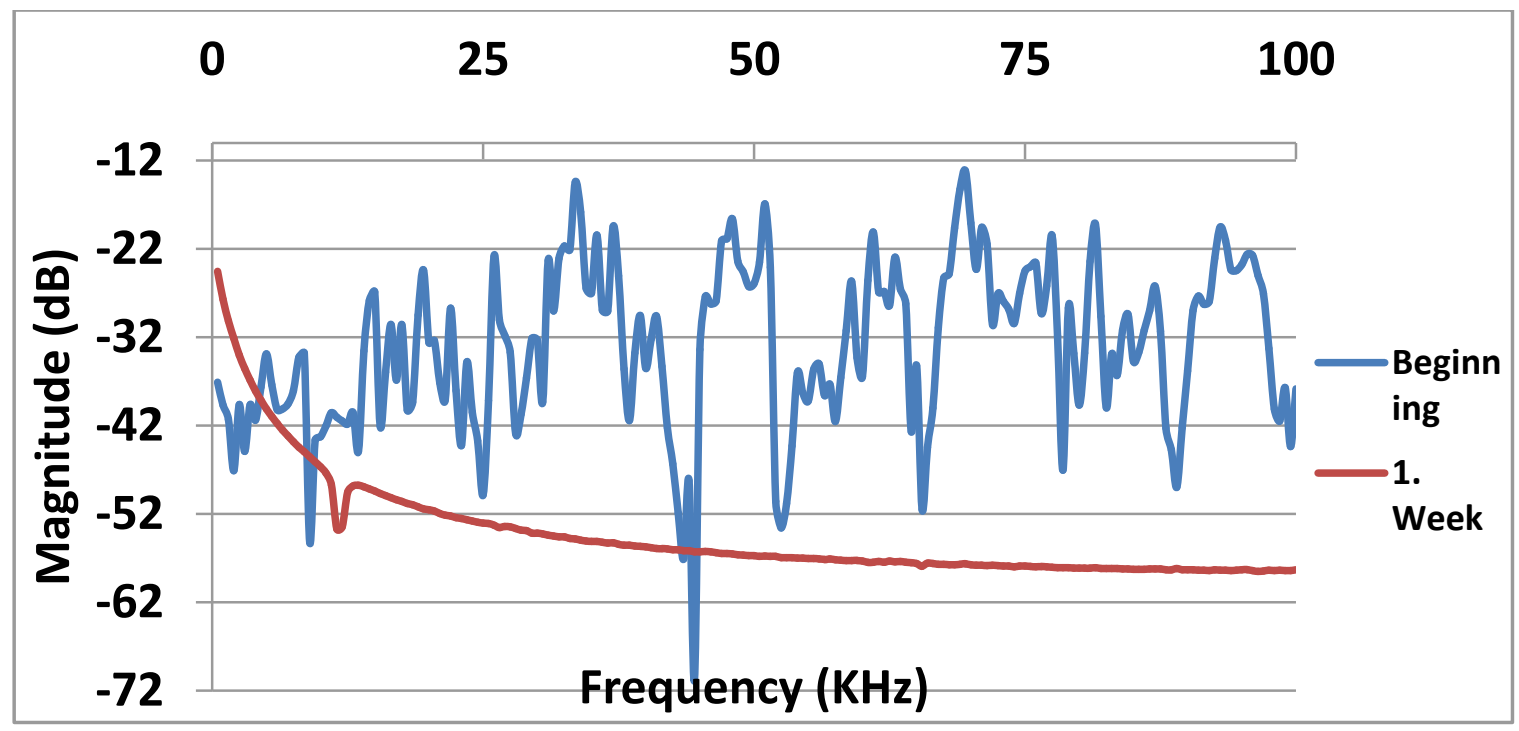

Figure 3.13 Frequency Response of the APC D-018 piezo of the Plate 1

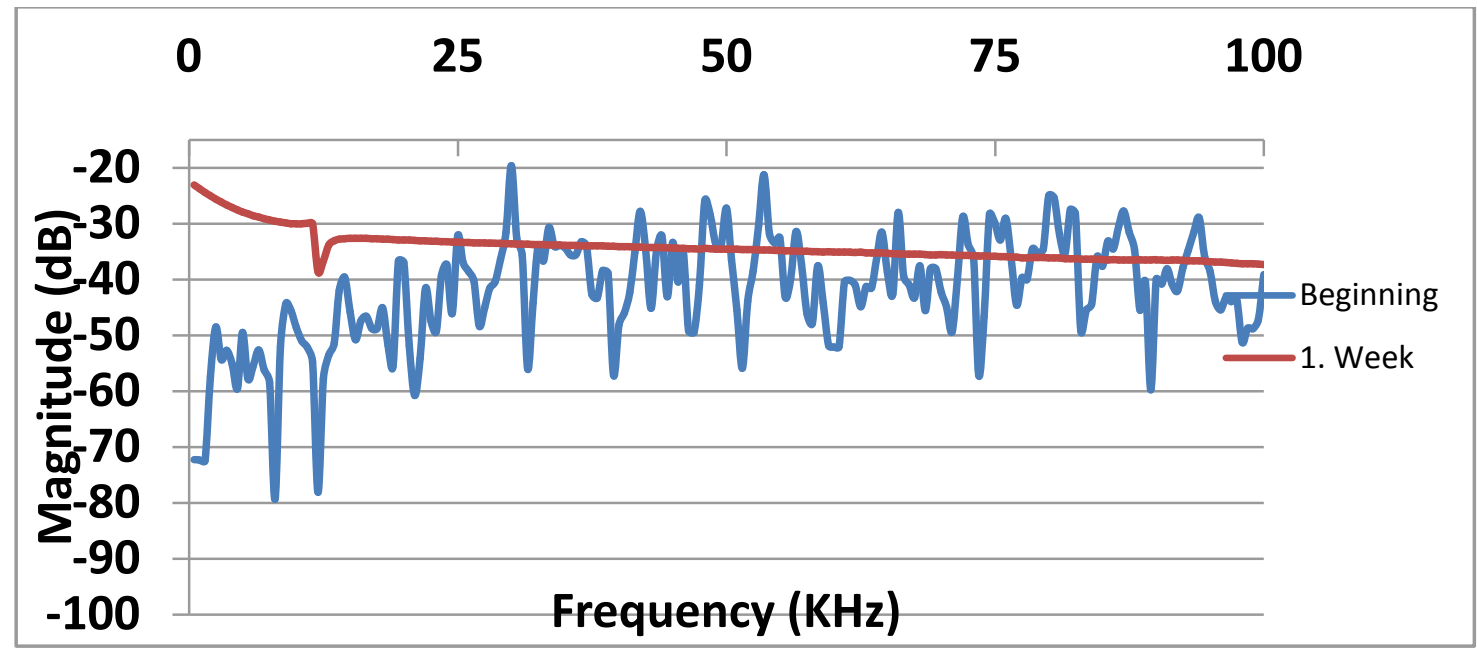

Figure 3.14 The Frequency Response of the Metis MD1 of the Plate 2

Observing the above plots obtained from the data recorded from the experiment clearly shows that after first week of the experiment, there was no output signal from the piezoelectric sensor. The adhesive was pulled off from the surface of test plate (figure 3.12) after one week underwater, and suggests that the piezoelectric sensor bonded with MBond AE-10 deteriorates when they are under water. Hence, by comparing 
the results of the above two experiments it can be seen that M-Bond 200 performed better than M-Bond AE-10 adhesive for underwater conditions.

\subsection{Conclusion}

The endurance and sustainability of the piezoelectric elements and the bonding material used was analyzed by testing them under different conditions i.e. under room temperature, at elevated temperatures and under water condition. The piezoelectric elements at room temperature were working without any problem over 20 week period with or without external excitation. There was no significant change in the characteristics of the bonding material as well as the piezoelectric transducers/sensors used. Bonding material M-Bond 200 was first subjected to temperature range from $50^{\circ} \mathrm{C}$ for one week and $100^{\circ} \mathrm{C}$ the next week. The response characteristics started to change at the end of $8^{\text {th }}$ week but the adhesive immediately failed above $100^{\circ} \mathrm{C}$. On the other hand $\mathrm{M}-\mathrm{B}$ ond $\mathrm{AE}-10$ was tested at $100^{\circ} \mathrm{C}$ for one week and $200^{\circ} \mathrm{C}$ the next week. There was no visual degradation of the adhesive at that temperature, but the response characteristics indicated that the piezoelectric elements failed at $200^{\circ} \mathrm{C}$. The results for underwater conditions were opposite to the previous condition. The response characteristics of the piezoelectric couples which were attached to the aluminum plate with M-Bond 200 and located under water showed significant changes at the weekly inspections. At the end of the $8^{\text {th }}$ week, there was significant change compare to the beginning but piezoelectric elements were still operating and there was visually noticeable deterioration. On the other hand M-Bond AE-10 adhesive 
became nonfunctional within one to two week period. From the above discussion, it can be concluded that both adhesive and piezoelectric elements perform good under room conditions. For high temperatures, MBond AE-10 performs much better that M-Bond 200 whereas for underwater condition, opposite response was observed. 


\section{CRACK GROWTH MONITORING}

\subsection{Literature Review}

Health monitoring and damage detection of structural and mechanical systems utilizing vibration measurement have attracted much attention in the past few decades. Most crack detection methods were tailor made for one-dimensional beam type structures [49-50], simple shear building models, trusses, two-dimensional frame structures and three-dimensional frame structures [51-54]. Early work related to cracked plates focused mainly on the problem to investigate the vibration and stability problems of the cracked plate. There is little research on crack detection in plate type structures. For instance Cawley and Adams [55] proposed a method to locate and roughly quantify defects on a rectangular plate based on finite element method utilizing changes in measured natural frequency in 1979. In 2000, Chen and Bicanic [56] developed a method to detect damage in a cantilever plate using both natural frequency and mode shape based on FE analysis. Later Yam [57] investigated the sensitivities of static and dynamic parameters of damage occurring in plate like structures based on FE model, and corresponding damage indices were proposed to analyze their identification capabilities. It must be pointed out here that it is difficult to model a part-through crack using the FE method owing to the narrow gap and complex shape of the crack [58-]. As a result, FE solution for this type of cracked plate analysis has a high computational cost. 
Many studies have also contributed to damage detection based on Lamb wave propagation in thin metallic structures [60]. Representatively, Tua et al. [61] located cracks in cylindrical pipes and plates with piezoactuated Lamb waves, and discussed the capacity for detection of cracks filled with impurities. Ihn and Chang [62] monitored hidden fatigue crack growth using a damage index (DI) with a built in piezoelectric sensoractuator network. In many studies for crack identification, the crack is generally perpendicular to the propagation path of Lamb waves or the crack is infinite at one end. As a result, the interaction between Lamb waves and the crack is usually simplified into normal transmission and reflection only.

The quantitative relationship between Lamb wave scattering and crack length can usually be described by the reflection and transmission coefficients. Two approaches are normally utilized to calculate these coefficients in frequency or time domain. In the frequency domain, the frequency spectrum of the reflected or the transmitted signal can be divided by that of the incident wave signal to obtain the reflection or transmission coefficients as a function of frequency. Alternatively, these coefficients can also be obtained by dividing the value of the reflected or transmitted wave peak by that of the incident wave peak in the time domain after applying Hilbert transformation (HT) [63].

In this chapter, elastic behavior of an aluminum plate was studied for monitoring the crack growth. The propagation of the Lamb waves and 
surface excitation response was analyzed. Both approaches used the same piezoelectric sensors/actuators.

\subsection{Methodology and experimental setup}

The growth of a crack in an aluminum plate was monitored by using both Lamb waves and evaluating the surface excitation response. The surface excitation response approach is similar to well-known impedance measurement method; however, used a less expensive spectrum analyzer approach.

For Lamb wave analysis, one of the piezoelectric elements was excited by a burst of three square waves, and the propagated signal was acquired at the other piezoelectric element. The frequency of interest was isolated by using the s-transformation method. The resultant signal corresponded to the envelope of the excitation frequency waveform.

The surface excitation response was evaluated by actuating one piezoelectric element with a swept sine wave signal and measuring the voltage at the other one. Spectrum analyzer was used to generate the excitation signal, acquired the data, and calculated the frequency response of the system.

Both approaches proved to have significant damage detection capabilities. The envelope pattern and frequency response curves changed drastically as the crack length increased. These sensitive detection techniques could be monitored by calculating the sum of the squares of the 
difference between the baseline data and subsequent damage cases. This study indicates that Lamb wave analysis and evaluation of the surface excitation are effective methods for monitoring the crack growth.

An aluminum plate specimen of $12 \times 6 \times .25$ inch dimensions was used. Several approaches were used to create a crack at one of the edges of the plate. Since a large amount of pressure is required to enforce a crack on a straight edge, a notch was first developed at one of the edges of the plate to increase the stress concentration at that point. After a notch was created, the plate was first restrained in two brackets from sides and then a vertical force was applied on those brackets with the help of a hydraulic press (figure).

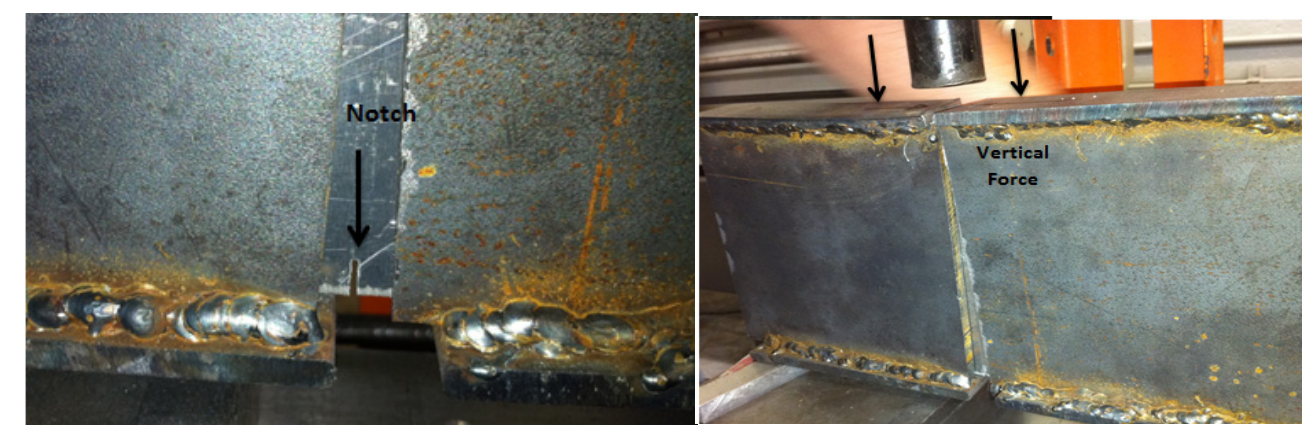

Figure 4.1 Process of crack formation

A crack was then created successfully by the above procedure without much damage to the plate. After an initial crack, two APC international (D-0.25 inch) piezoelectric actuators were bonded to the plate using $\mathrm{M}^{-}$ Bond 200 adhesive (Figure 4.2) and data was recorded using both Lamb wave \& surface excitation to response techniques. Furthermore, the crack was increased until the plate was torn apart into pieces. Before the 
separation of plate, the data was taken at three different stages of the crack and the length of the crack at these stages was 1.0, 1.5 and 2.0 inch (figure 4.3).

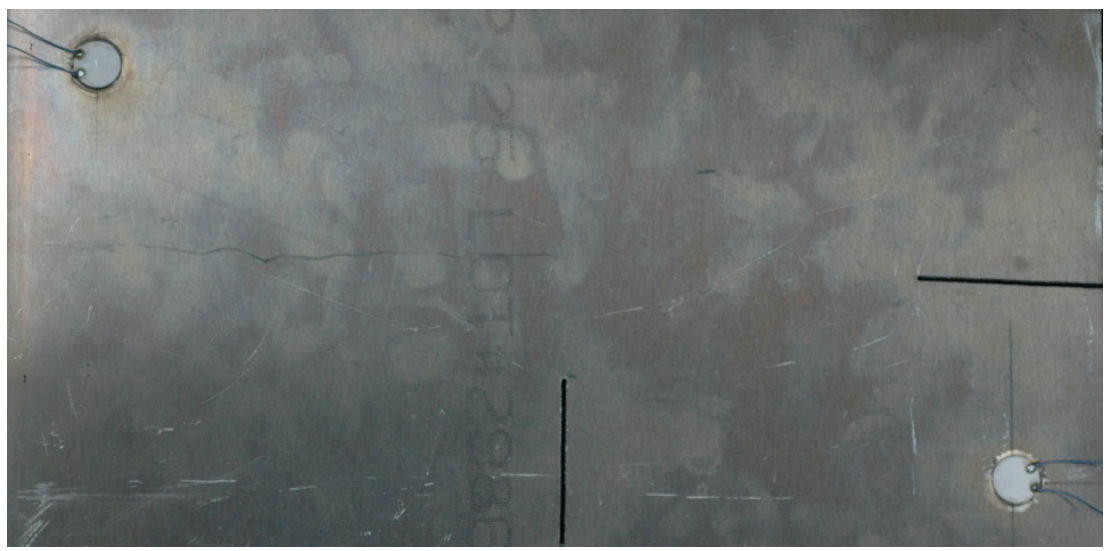

Figure 4.2 Aluminum plate with initial crack.

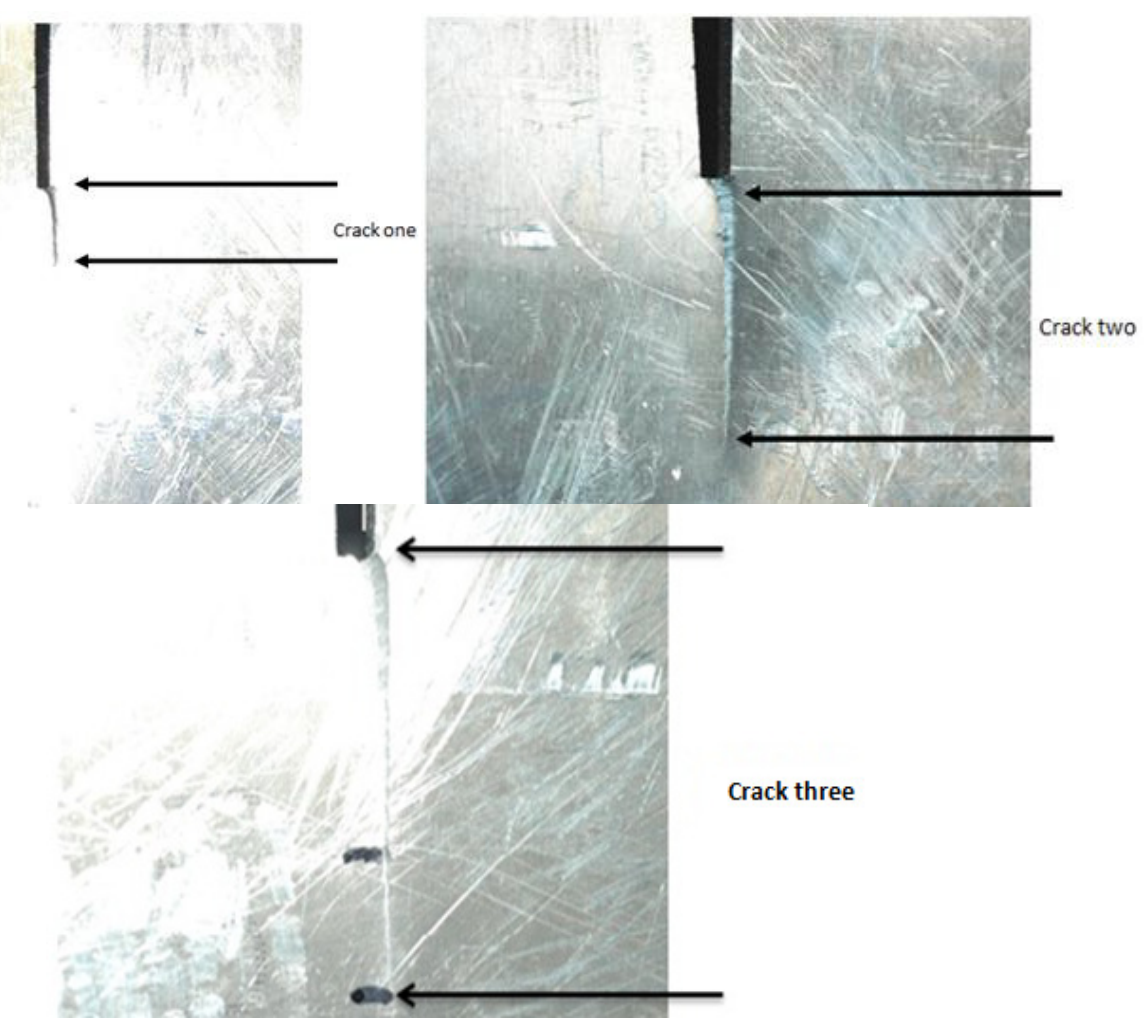

Figure 4.3.The three stages of the crack on the aluminum plate. 


\subsection{Data Acquisition}

To monitor the crack growth in aluminum plate, two approaches: Lamb wave analysis and surface frequency response to excitation were used. An aluminum plate sample bonded with two APC international (D-0.25inch) piezoelectric elements at the opposite corners of the plate (figure 4.2) was used for analysis. The location of the sensors was chosen to be at the corners, in order to scan the entire plate for defects.

For the Lamb wave analysis, an amplified burst of three square waves with excitation frequency of $190 \mathrm{KHz}$ was applied to the actuator. A PicKit 2 microcontroller board circuit was built and programmed to generate the square waves with the required frequency. The signal from the microcontroller was transmitted to one of the piezoelectric elements called as the actuator through a power amplifier (TEGAM - 2348). The voltage of the other piezoelectric element called as the receiver was measured by using Tektronix TDS-2024B digital oscilloscope (figure 4.4). In this way a total of three set of data was acquired for three different crack lengths and further the data was analyzed to monitor the damage. 


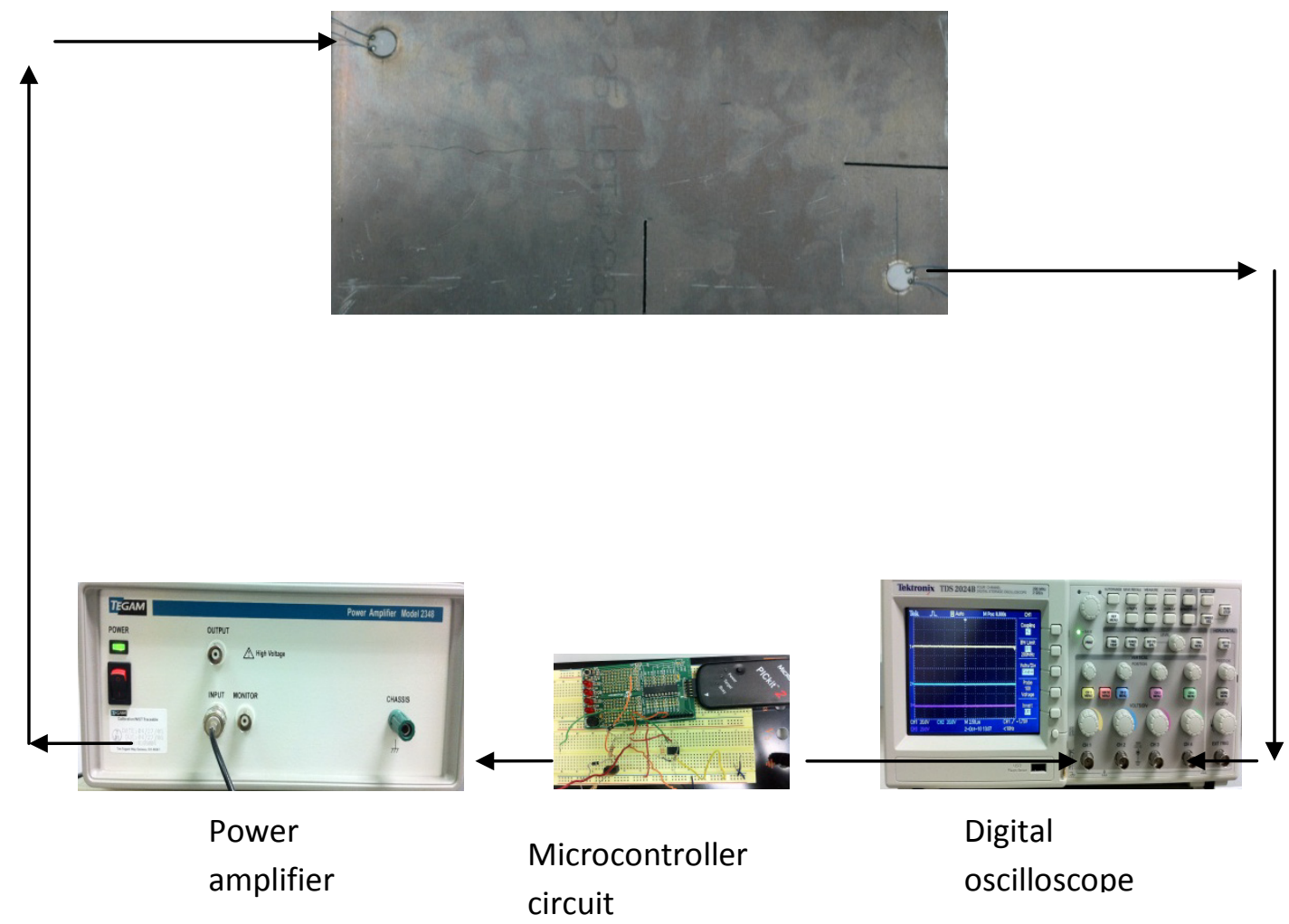

Figure 4.4.Outline of the experimental data collection (Lamb wave approach)

For frequency response of surface upon excitation, same sample was used. In this approach, one of the piezoelectric elements (actuator) was excited with a continuous sweep sine wave of frequency range from 1-100 $\mathrm{KHz}$, generated by the Stanford Research Systems (SRS) -SR780 spectrum analyzer. While the data was recorded from the other piezoelectric element using the same spectrum analyzer, that also monitored the voltage of the other piezoelectric element (figure 4.5). Similarly, three set of data was recorded for three different crack lengths. 


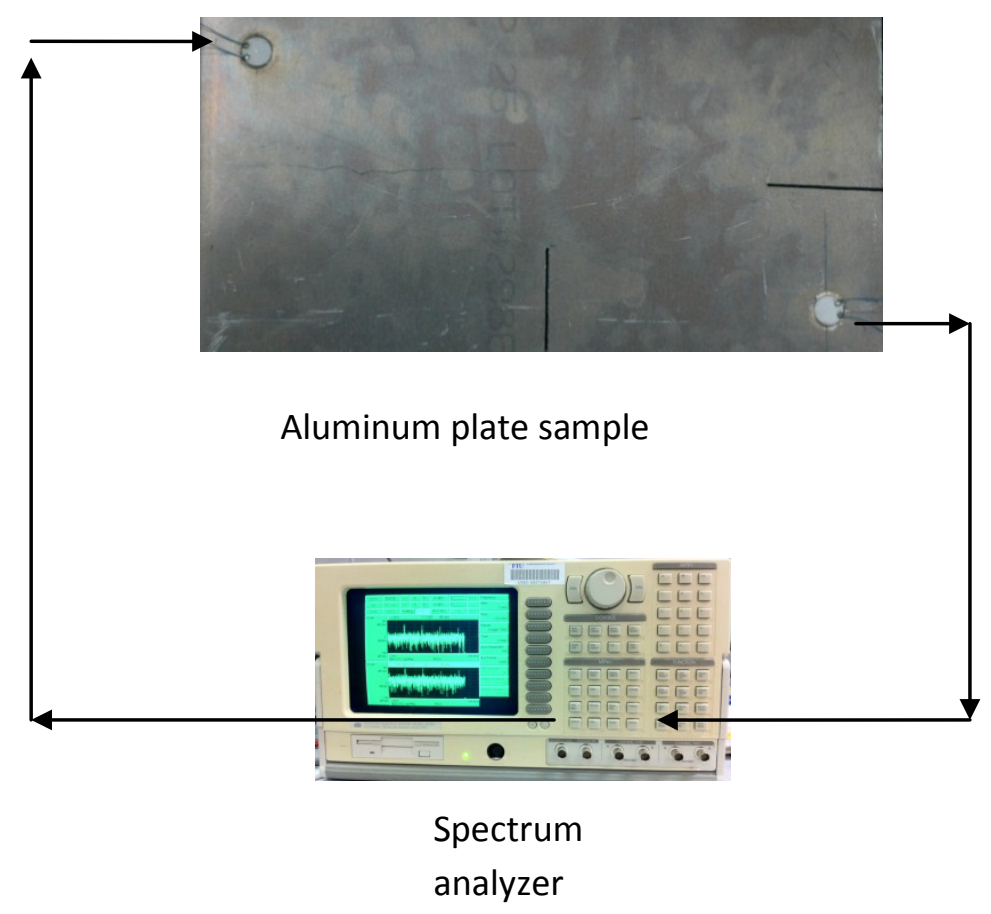

Figure4.5 Outline of the experimental data collection (Frequency response to excitation approach)

\subsection{Data Analysis}

For the analysis of the Lamb wave method the S-transformation of the signal was calculated, and the excitation frequency was determined. Since it was difficult to determine the exact change in the behavior of signal response upon change in structure properties, the magnitude only at the excitation frequency was isolated and plotted. This signal basically represents the envelope of the sine waves at the excitation frequency. The followed procedure is presented in figure 4.6. 


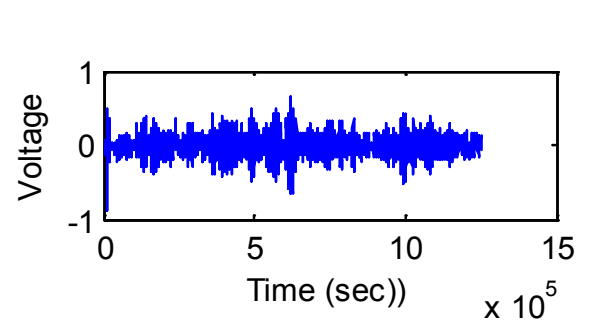

Acquire data in time domain

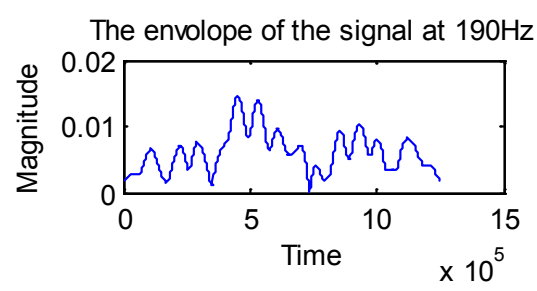

Taking magnitude values

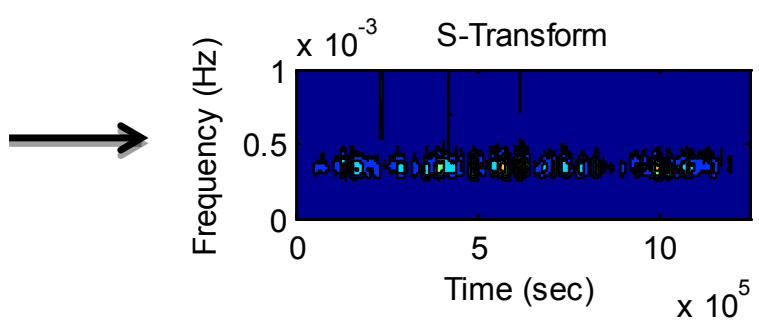

Take S-transformation of data
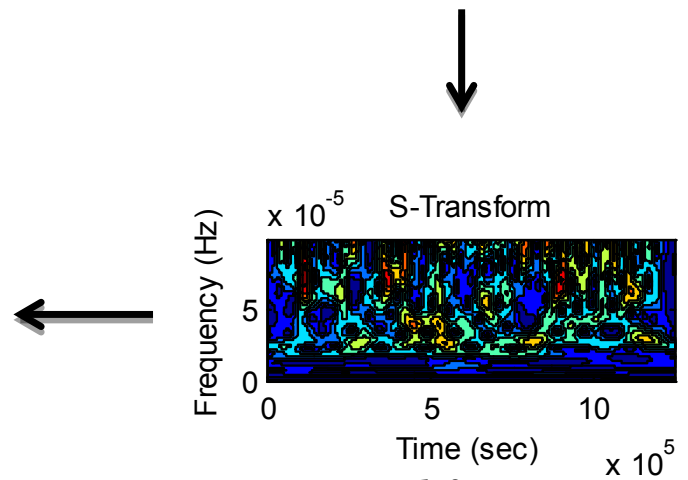

Focus on interested frequency

Figure 4.6 Analysis of the Lamb wave data by using the Stransformation

In second approach, the frequency responses of the surface response to excitation at different crack lengths were obtained and the sum of the squares of the difference from the baseline data was obtained and the results were visualized using a bar graph. Since a notch was first created in order to initiate a crack, the small crack that was created first was considered as the baseline data and the other two cases were compared to it.

\subsection{Results and discussion}

Crack growth in an aluminum plate was monitored using two approaches. The first approach created Lamb waves on the surface and monitored their propagation. The experimental signals at the three different crack lengths are presented in figure 4.7. Clearly, the envelopes 
of the signals were very dissimilar and a significant difference can be seen by comparing these signals, but the magnitude and the intensity of the defects cannot be judged from these experimental results obtained. Hence to analyze the signal characteristics better, the S-transformation of the signal was calculated and plotted in figure 4.8. The S-transformations were also very different. To simplify the interpretation of the timefrequency plots and to identify the intensity of damage, the envelope of the signals were generated at the excitation frequency about $190 \mathrm{KHz}$ and presented in figure 4.9 .
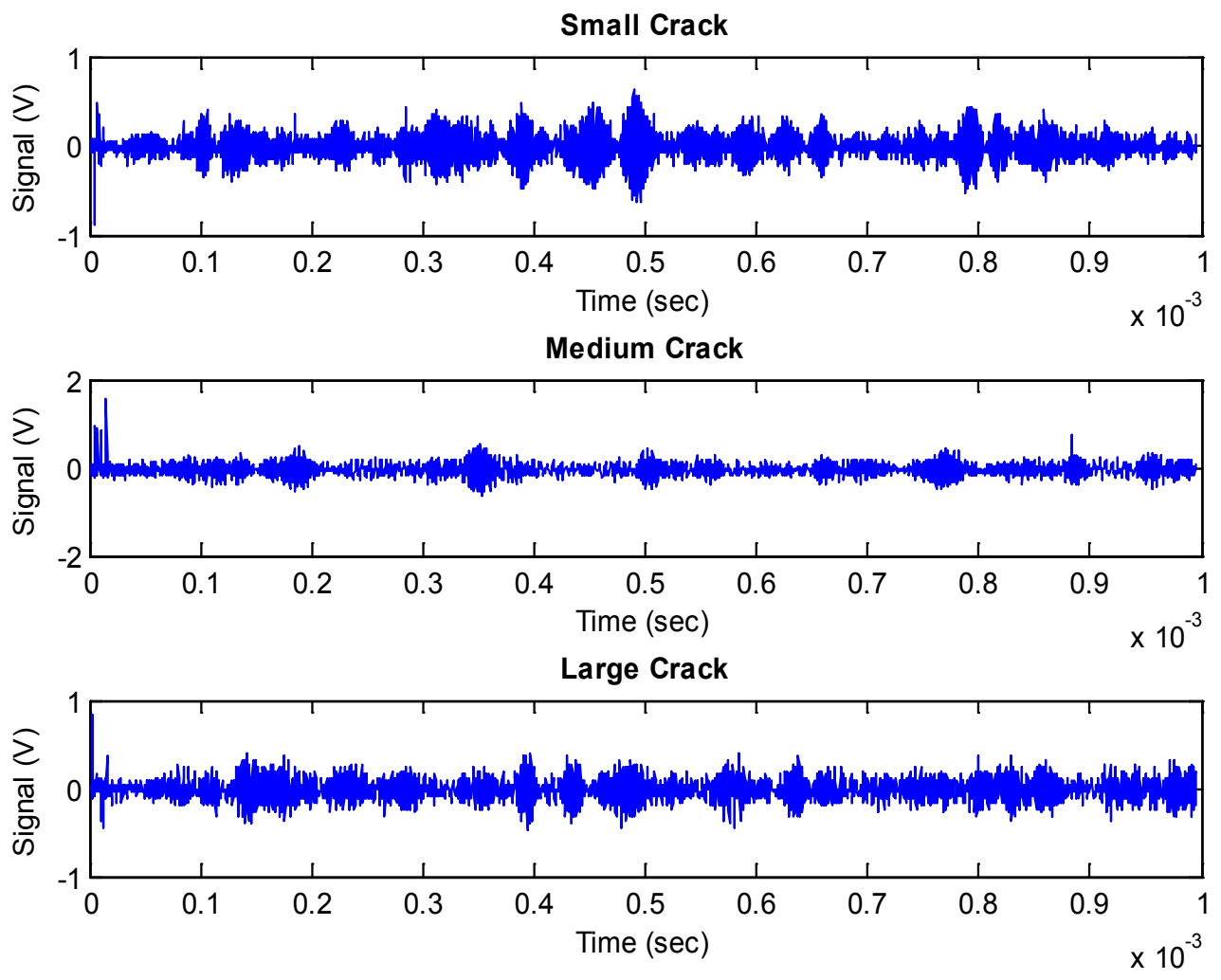

Figure 4.7.The voltage variation at the sensing piezoelectric element after the Lamb wave was generated. 

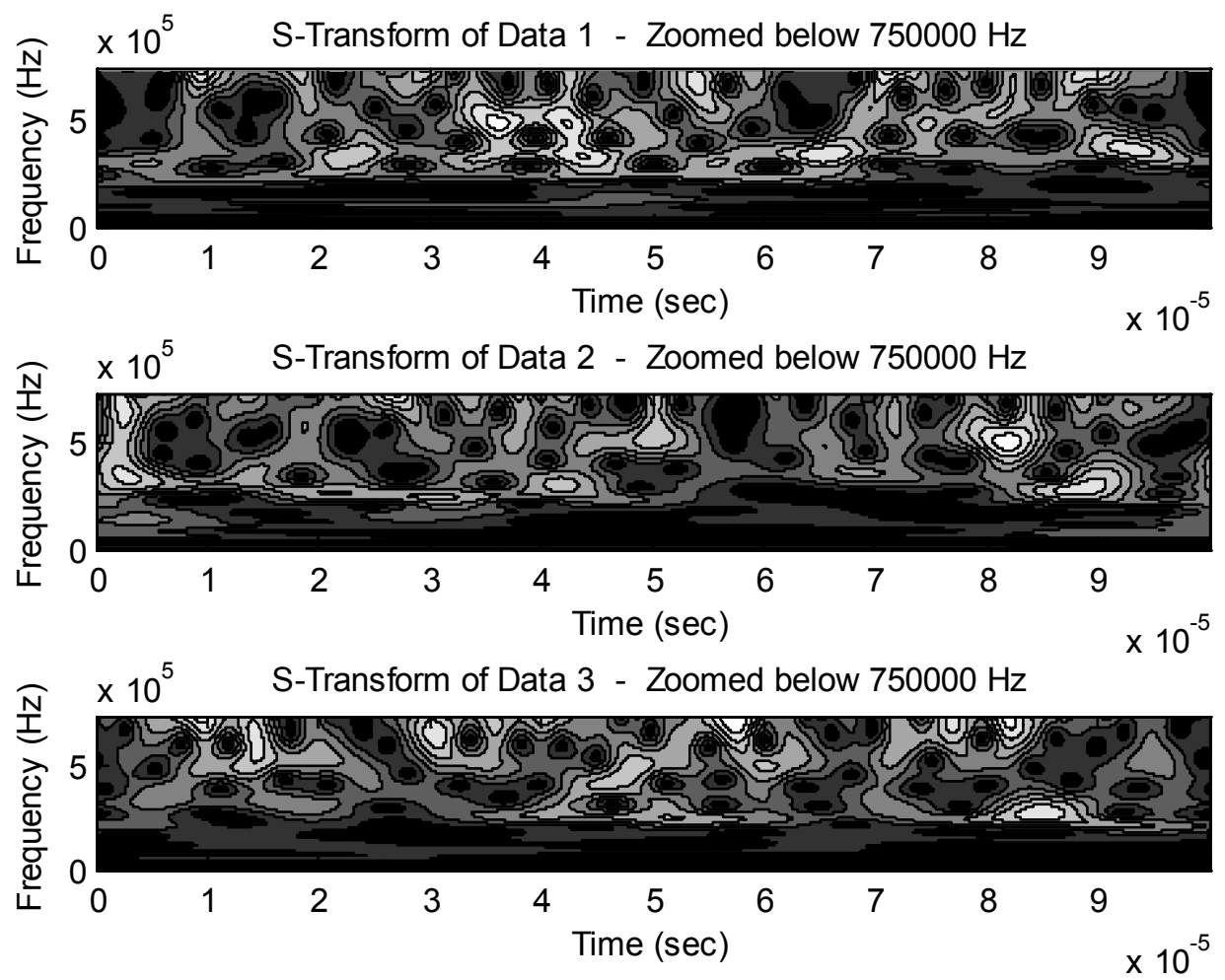

Figure 4.8.The S-transformation at the interested frequency range.

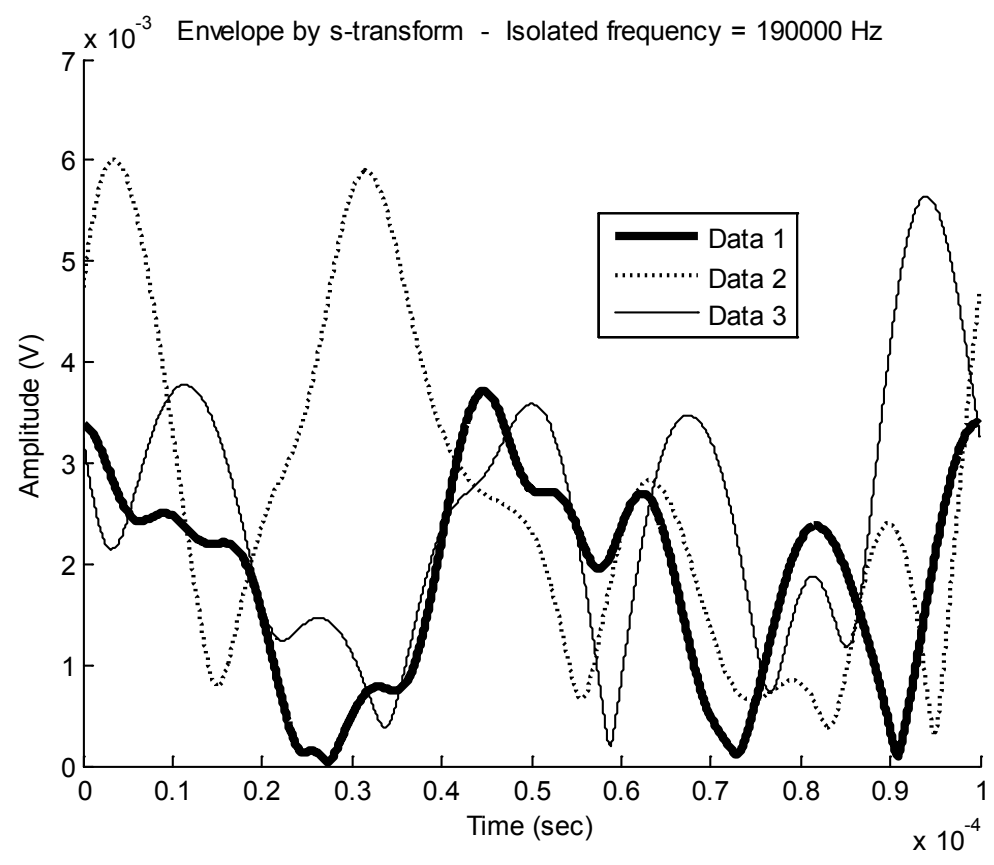

Figure 4.9 Comparison of the envelopes of the signals when the crack had three different lengths. The crack length was 1", 1.5", and 2" in the Data 1,2 , and 3 respectively. 
From the envelopes obtained at the excitation frequency, it can be seen that between 60 -70microsecond time, the amplitude of data 3 (large crack) increased significantly as compared to data 2 (medium crack) and data 1 (small crack).

The frequency response characteristics of the surface response to excitation were also evaluated. One of the piezoelectric elements was excited by sweep sine wave and the magnitude of the measured signal at the other piezoelectric element was calculated by using a spectrum analyzer and plotted (figure 4.10). The characteristics of the signals were very different. To understand the difference between the signal responses and to visually detect a meaningful change in the characteristics of the signal, the square of the difference between the three test cases was calculated (figure 4.11). 

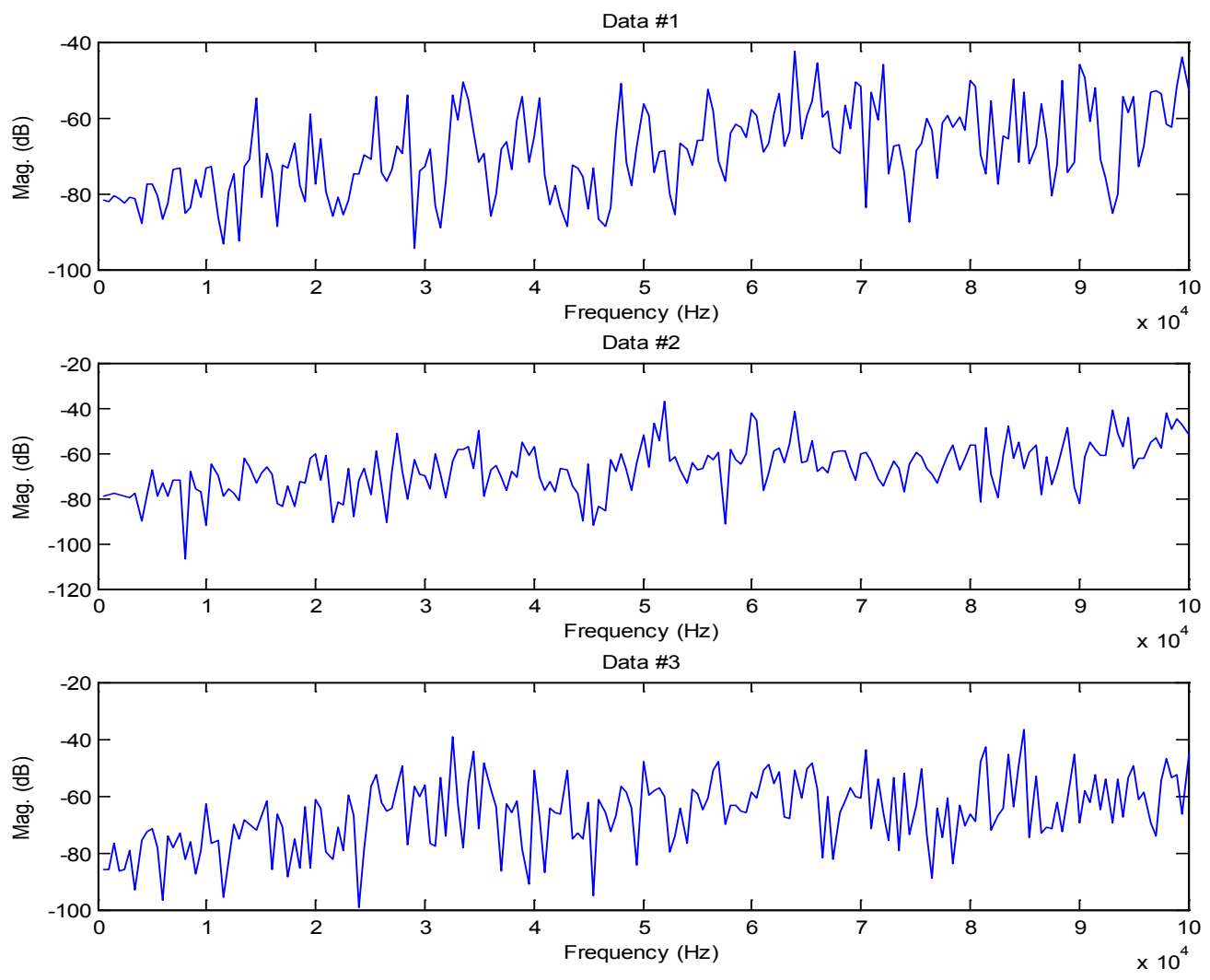

Figure 4.10.The measured frequency response characteristics at different crack lengths
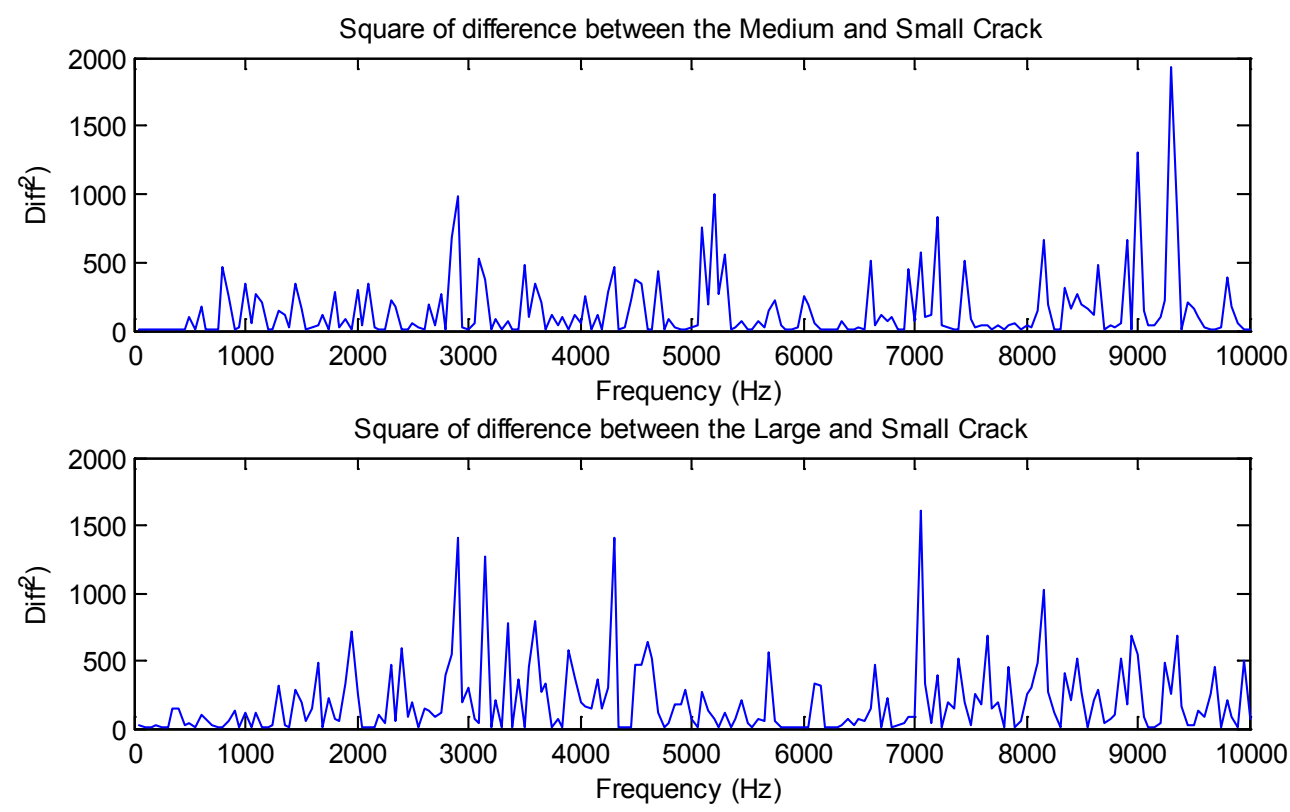

Figure 4.11.Square of difference of the magnitude of signals 
From the above obtained plot after calculating the square of difference of the magnitudes of the signals received by the signal analyzer, an indicating difference was noticed at around $70 \mathrm{KHz}$ frequency. But an exact amount on increase in the intensity of defect from small crack to large crack cannot be determined from these plots. Hence, the sum of the squares of the differences at each frequency was calculated (figure 4.12) and the differences were presented in the form of bar graph. The frequency response characteristics changed each time when the crack length increased and the magnitude of the difference increased with the increase in the crack length.

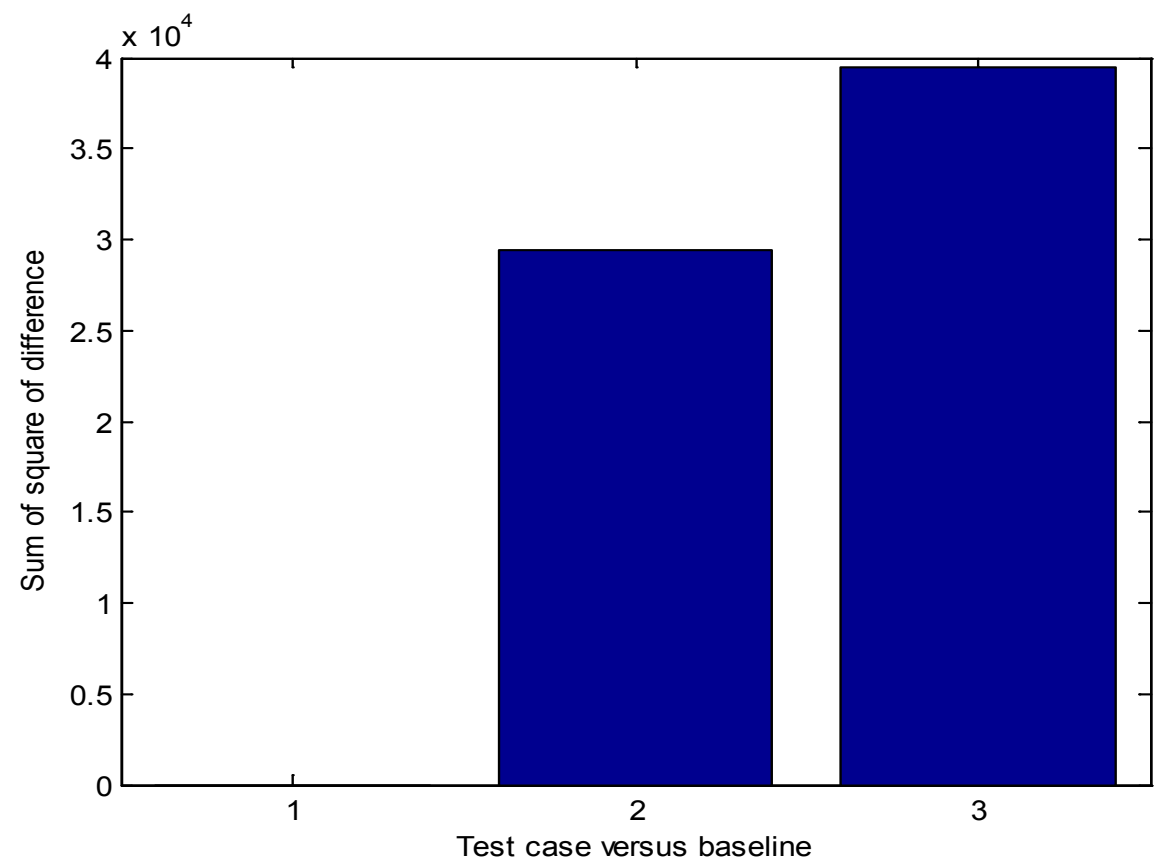

Figure 4.12.The sum of square of the difference between the first and second; and first and third frequency response characteristics. 


\subsection{Conclusion}

In this chapter, Lamb wave propagation and SuRE were evaluated to monitor the growth of the crack length. The Lamb wave approach compared the envelopes of the sensory signals after they were obtained by the S-transformation. The SuRE method calculated the frequency response characteristics of the surface by using two piezoelectric elements.

The envelopes of the received signals after Lamb waves created at the surfaces and the characteristics of the SuRE to oscillations drastically changed with the growth of the length of the crack and indicated that both approaches could be used for monitoring the integrity of an aluminum plate. Both approaches used the same sensors and complemented each other. The sum of the squares of the differences of the frequency response characteristics indicated the severity of the crack. This approach was very easy to implement. The estimation of the crack length from the envelopes of the Lamb wave was not as straight forward as comparing the frequency response characteristics.

Studying the frequency response characteristics of the SuRE was similar to the impedance method. The number of the necessary sensors increased but the complexity of the measurement device significantly simplified from impedance analyzer to spectrum analyzer. 


\section{MONITORING THE DEFECTS IN WELDED PLATE JOINT}

\subsection{Introduction}

Conventional non-destructive inspection of welding joints is performed using radiographic inspection, liquid penetration inspection and ultrasonic methods. Liquid penetrant method is comparatively fast, economical and requires minimum technical skills, but can only locate surface defects without any indication of the intensity of the damage. Radiographic inspection method on the other hand can detect internal flaws, but the main limitations are location and intensity of defect, moreover this technique requires very expensive equipment, high degree skill and experience, and has safety hazard associated with the use of radiations. Ultrasonic methods are used to locate weld defects and to determine their size. However, this technique requires surface preparation, and the use of coupling medium (oil, gel, etc.) to guarantee a high transmission of the ultrasonic beam and to facilitate the transducer scanning. The technique is not effective for fast scanning and when surface to inspect is irregular $[64,65]$. With ultrasonic immersion techniques the acoustic energy is transferred to the component dipping it completely into a water tank together with the transducers; even if fast, it is limited to small parts and cannot be used in manufacturing processes. Inspection operations together with the required equipment make the procedure time consuming and costly. 
Many studies have been published on laser based detection [66]. These methods are more precise and can represent a defect on highly reflecting surface, but are more complex and require higher investments. In today's world, there is an increasing pressure for cost-effective weld inspection. Cost-effectiveness is linked to factors such as reliability, sensitivity, speed and coverage. The need for greater speed, reliability and coverage has resulted in the increasing use of feasible and flexible systems for weld inspection.

Lamb wave and surface response to excitation approaches using piezoelectric wafer active sensors (PWAS) compared to conventional ones, is more economical, quick and reliable. The inexpensive sensors and equipment make this approach more popular worldwide. Lamb waves, allow inspection of the complete thickness with only one scan detecting both internal and surface defects. Lamb waves can be used in various practical applications for the high flexibility of measurement. As these waves travel at high speed and has low attenuation rate, a large area can be scanned for any defects in a very short time period

The aim of this work is to authenticate the applicability and the effectiveness of surface response to excitation and Lamb wave approach for quick and precise defect detection and convenience to use Lamb wave inspection for a reliable characterization of weld defects. 


\subsection{Experimental Setup}

To monitor the defects in a welded structure, a test specimen was prepared by welding two aluminum plates of $3 \times 3 \times 0.25$ inch dimension. The plates were welded with a butt joint, and three piezoelectric transducers were bonded on the specimen with M-Bond 200 adhesive. Out of these three transducers, one of the transducer was acting as an actuator and the other transducers were the receivers/sensors of the signal as seen in figure 5.1. The sensors were bonded on each side of the weld in order to receive and analyze the response to defects for each side. To monitor the response of the system due to a defect such as a cut or a hole in the welding joint, some defects were created into the specimen and their response was analyzed. A cut of 1/8 inch length and 1/25 inch wide was first made at the weld joint, which was further increased to $1 / 4$ inch length after the data was collected (figure 5.2), and a total of three holes of 1/16 inch were drilled with the help of drilling machine at three different positions on the test specimen (figure 5.3).

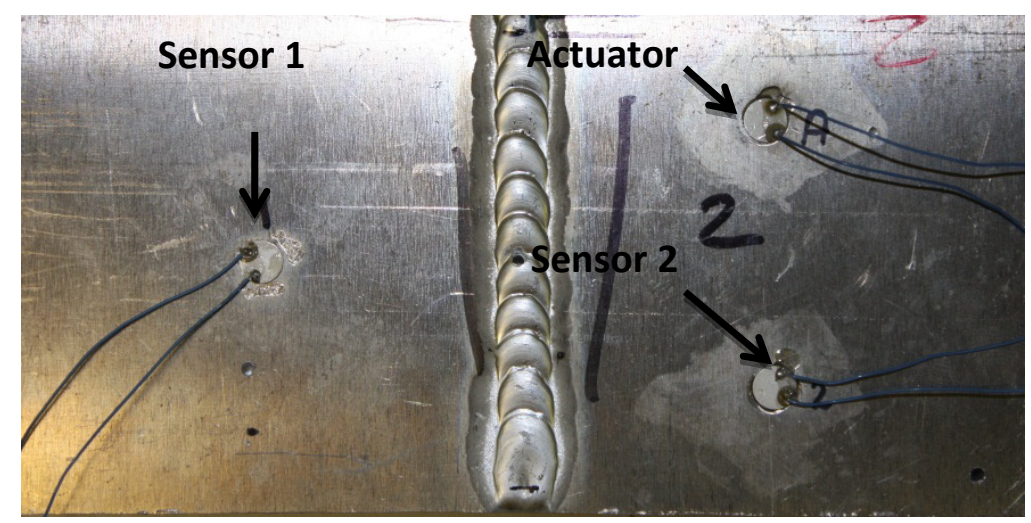

Figure 5.1 Plate specimen showing actuator \& sensors location 


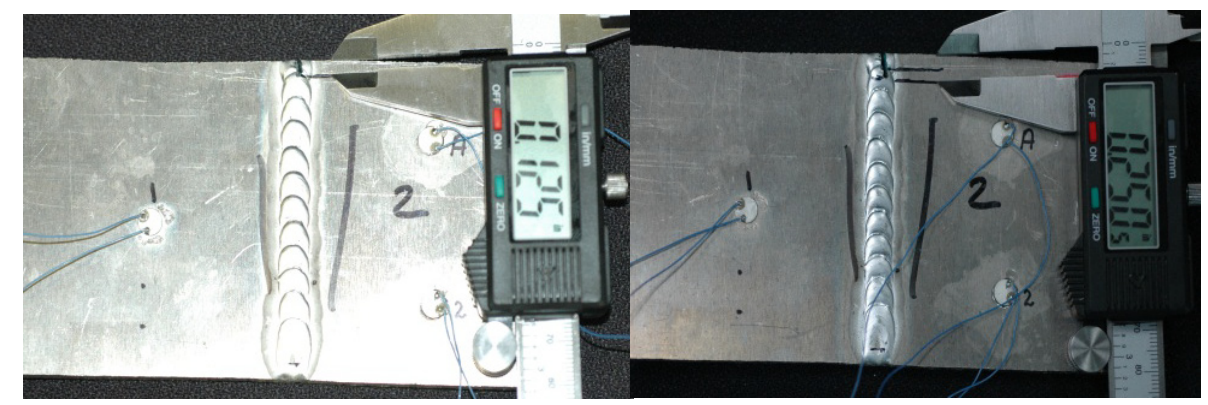

Figure 5.2.Weld plate specimen with two cut lengths $(1 / 4 \& 1 / 8$ inch)

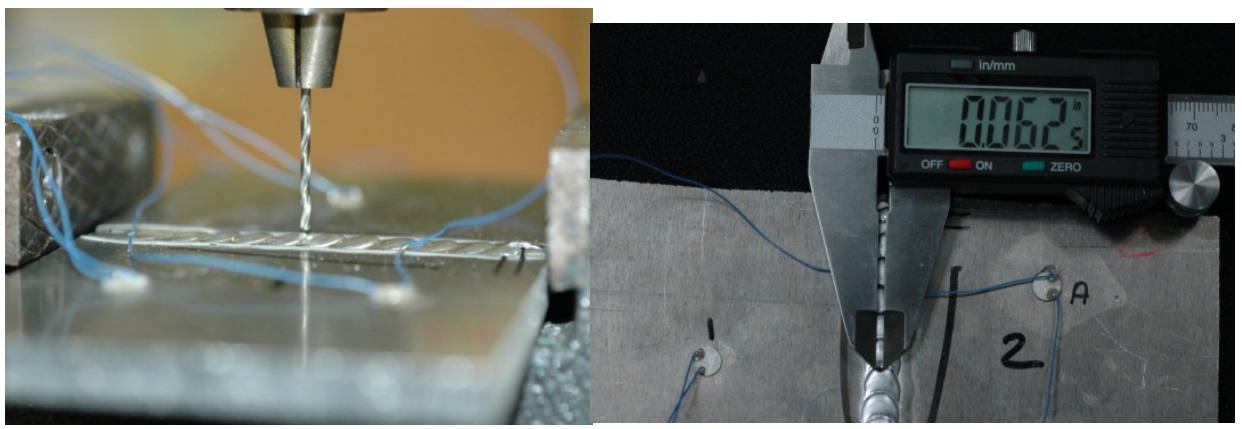

(a)

(b)

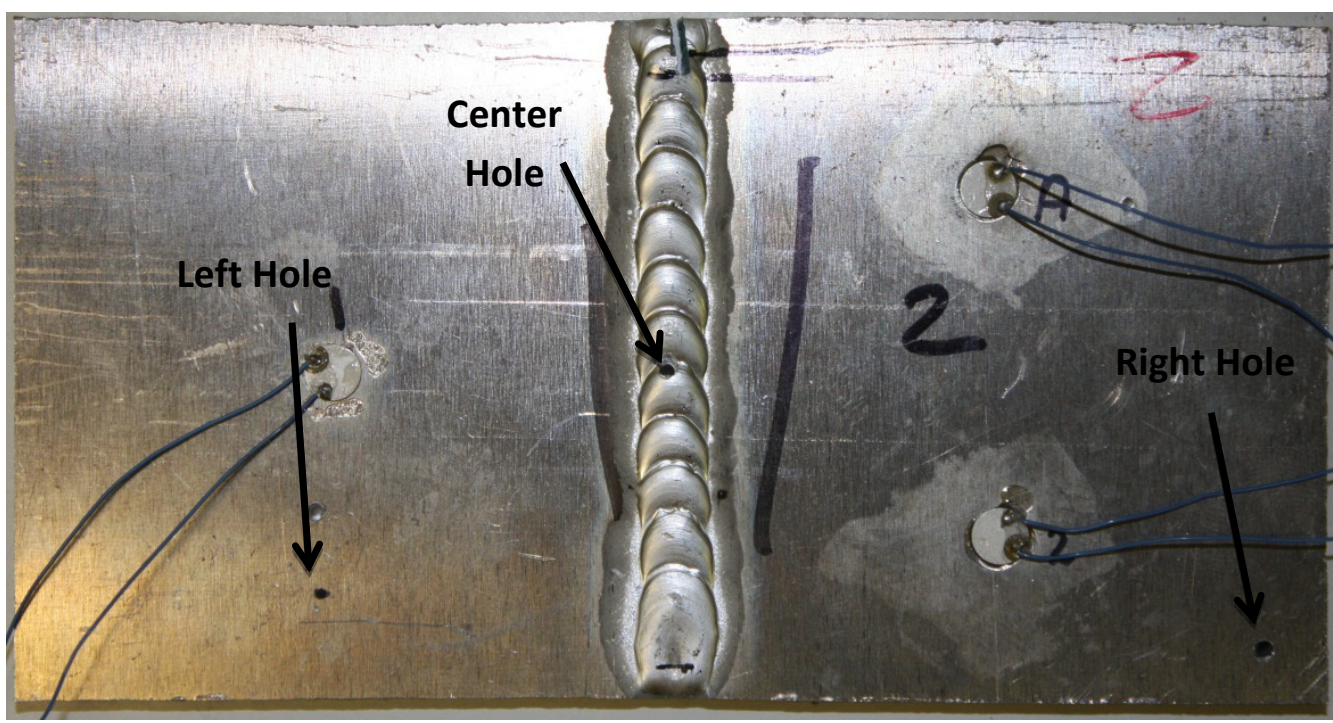

(c)

Figure 5.3 Welded plate sample (a) Drilling hole operation; (b) Showing hole diameter; (c) Figure showing positions of holes on sample 


\subsection{Methodology}

For Lamb wave analysis, the actuator on the specimen was excited by an amplified pulse generated by a digital oscilloscope, and the response of the signal was then attained at both sides of the weld through the sensors. A digital oscilloscope was used to record the data after each stage and this recorded data was then further analyzed to monitor and characterize the defect effectively.

In sweep sine wave approach on the other hand, the surface wave response of the structure to excitation is analyzed. A continuous sweep sine wave of frequency range $1-100 \mathrm{KHz}$ generated from a spectrum analyzer is used to excite the actuator, of the specimen and the subsequent response from the receivers/sensors is recorded. The signal analyzer is used for both generating the excitation signal and receiving the response of these generated waves to defects. Once the data from all defect conditions is calculated, it is further analyzed and compared.

\subsection{Results and discussions: Lamb wave approach}

The response of Lamb wave analysis is discussed under this section. Two types of defects (small cut/crack and hole) were monitored using two approaches. In first approach, Lamb waves were generated on the surface of the specimen and their relative response was monitored. In this defect analysis approach, a small cut/crack was monitored first, followed by the small holes. A total of three data sets was acquired and analyzed for three 
cases: perfect weld, small cut and enlarged cut on the weld. As there are a total of two receivers/sensors, one on each side of weld, we will refer them as sensor 1 and sensor 2 (figure 5.1). The experimental signals at sensor 1 $\& 2$ for the three stages are presented in figure $5.4 \&$ figure 5.5 . It can be clearly seen that the envelopes of the signal are very different from each other, but we cannot make a clear decision about the severity of defect. Since the experimental signal that we recorded was not isolated from surrounding noise and vibrations, we cannot conclude anything at this point.

Perfect

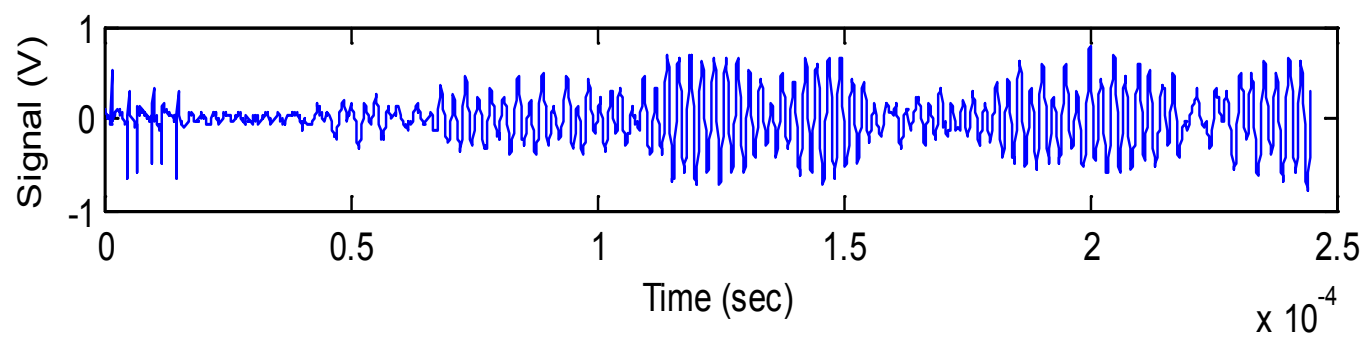

Small Cut

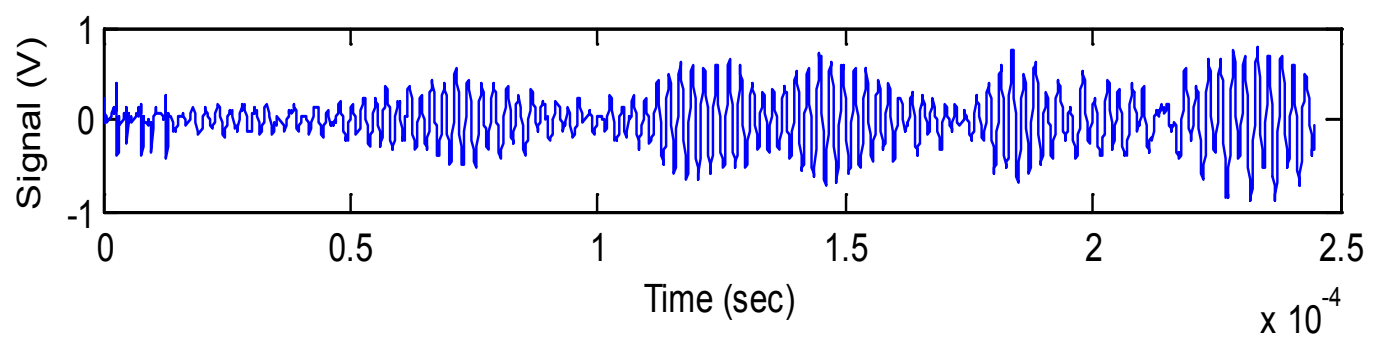

Enlarged Cut

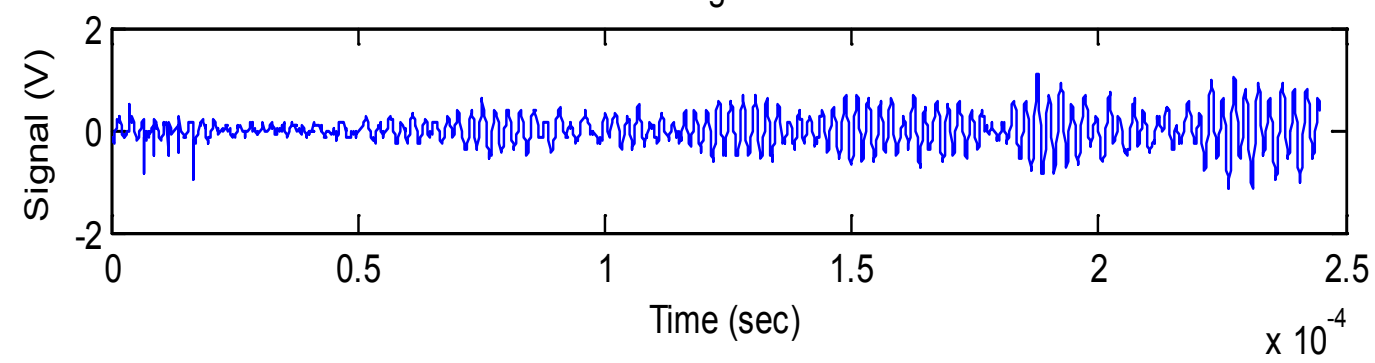

Figure 5.4 Lamb Wave response for three stages of cut at sensor 1 

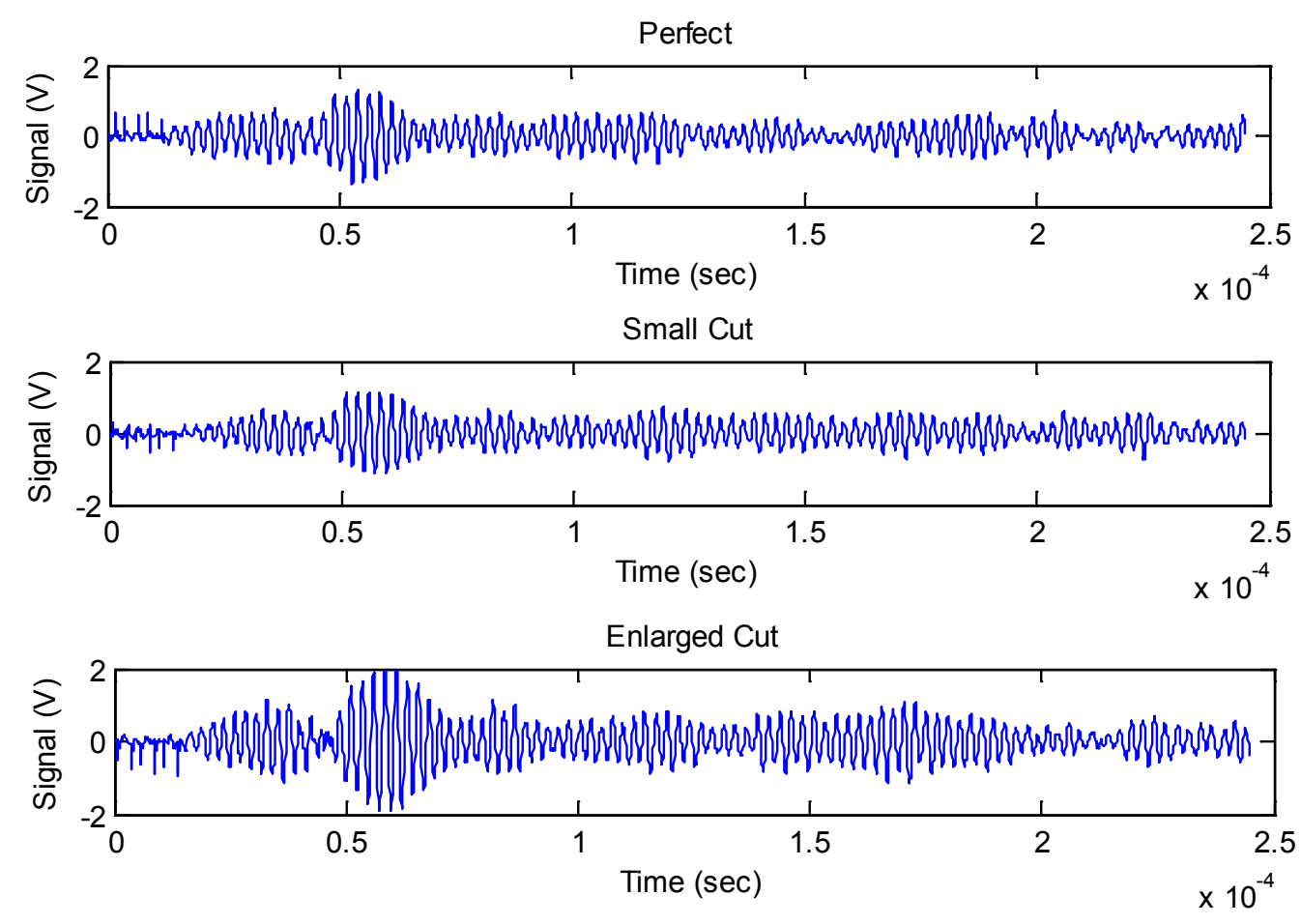

Figure 5.5 Lamb Wave response for three stages of cut at sensor 2

To isolate the signal from noise, vibrations \& unwanted reflections and to analyze the characteristics better, first the S-transformation of the signal was calculated, and then the envelopes of the signals were generated at the excitation frequency. The S-Transformation will allow us to determine the excitation signal frequency and hence simplifies fitting model in time domain. The S-transformations of the experimental signal are plotted in figure 5.6 \& figure 5.7. 


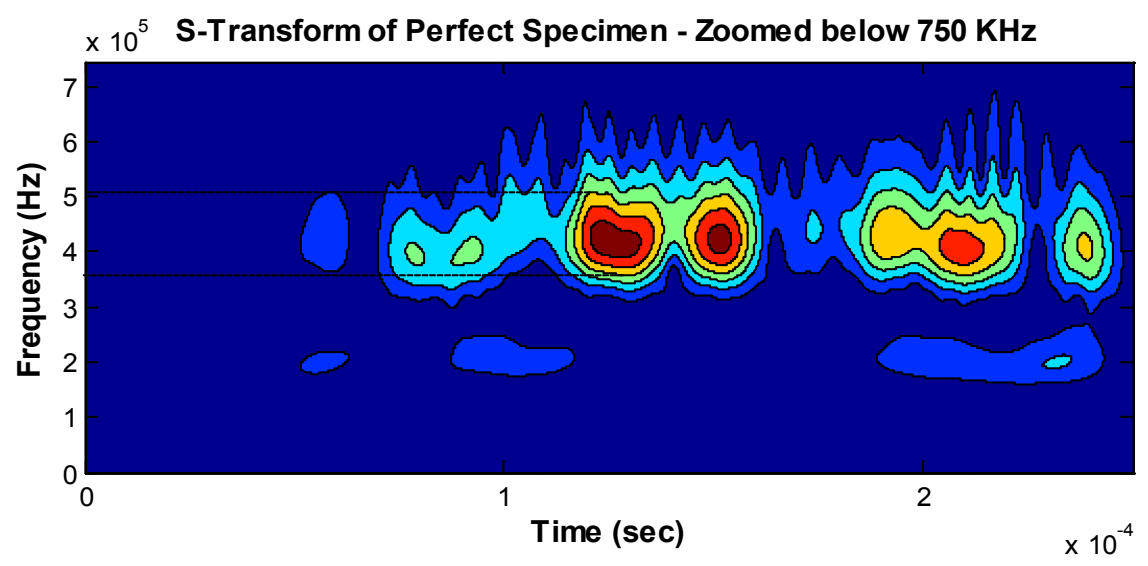

(a)

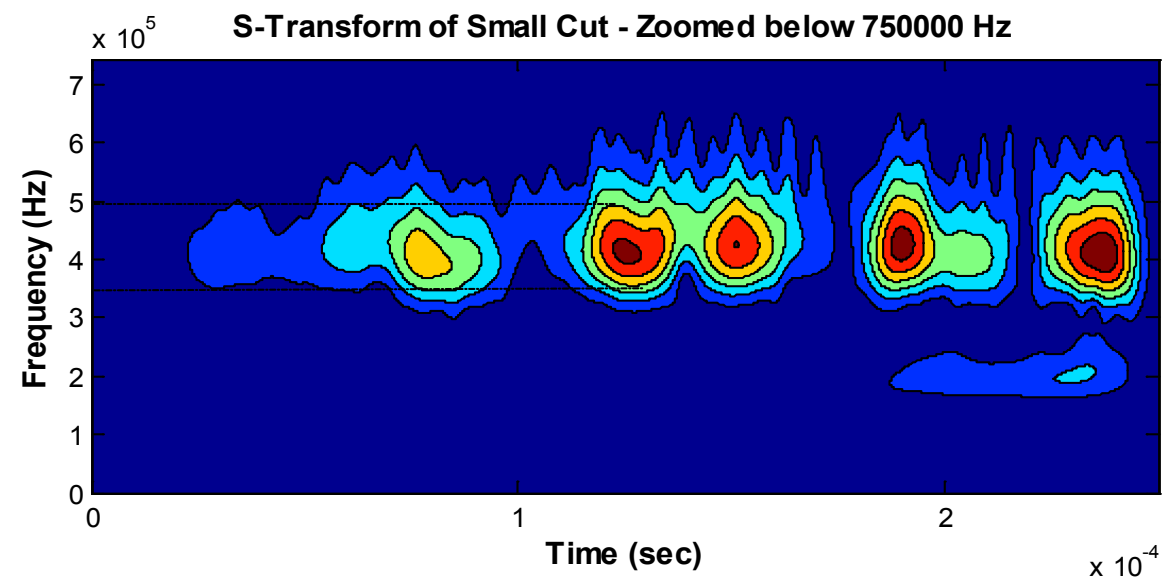

(b)

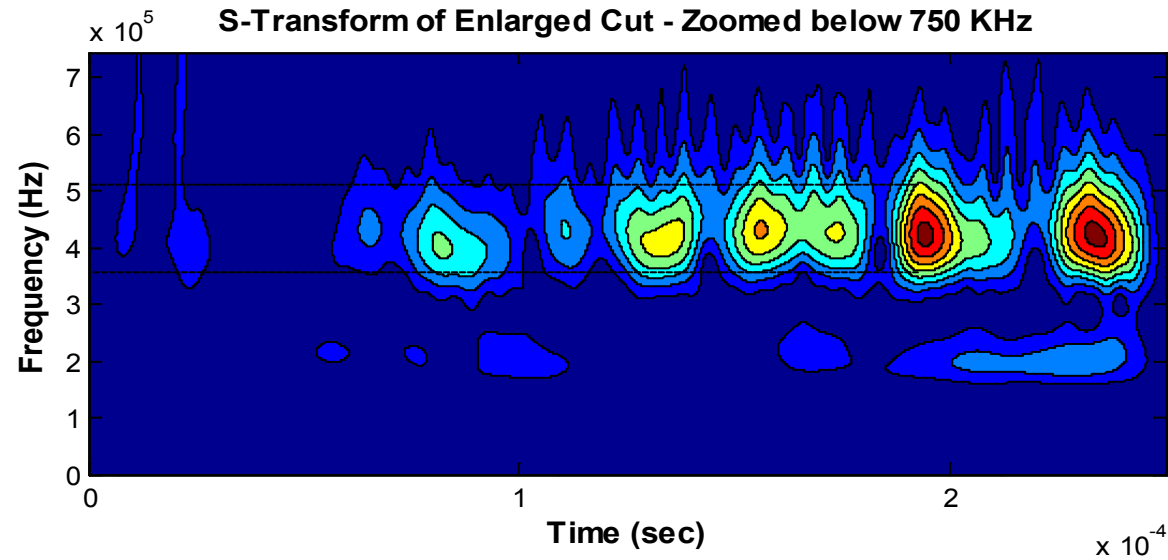

(c)

Figure5.6 S-transformation calculation at sensor 1 for; (a) perfect sample (b) small cut (c) enlarged cut 


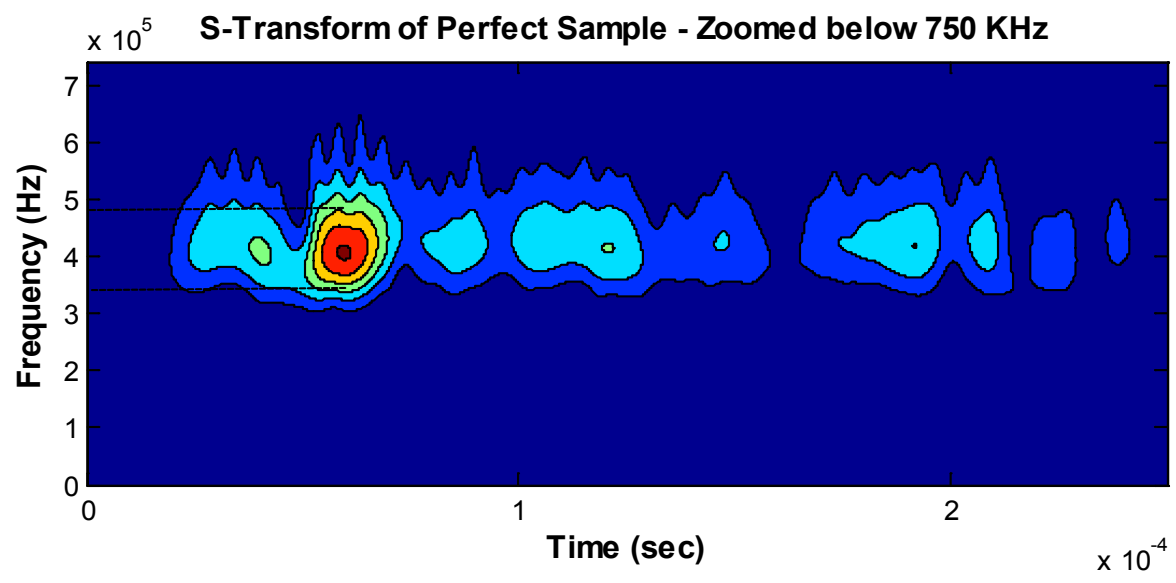

(a)

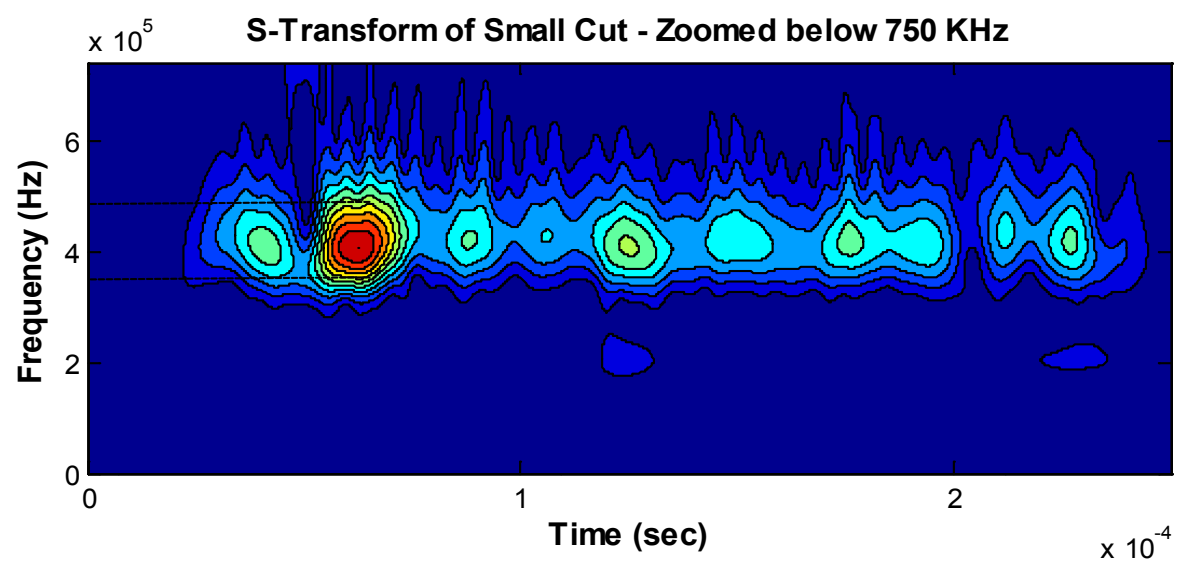

(b)

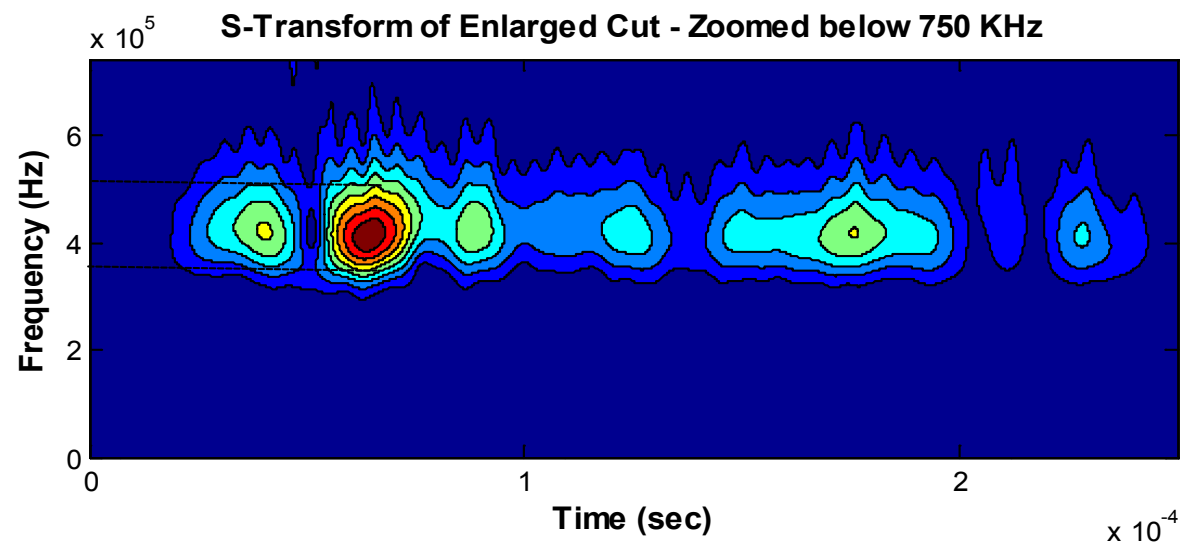

(c)

Figure 5.7 S-transformation calculations at sensor 2 for; (a) perfect sample (b) small cut (c) enlarged cut 
From the above obtained results obtained after S-Transformation, we can see the difference in the isolated signals. It can also be noticed that the excited signal from the actuator reached sensor 2 first as compared with that of sensor 1 because sensor 2 was located nearer than sensor 1 . As marked in the plots, the excitation frequency for all the cases came out to be between $400-500 \mathrm{KHz}$. After calculating the S-Transformation, the envelope of the signals was generated. The excitation frequency for the envelope was taken as $450 \mathrm{KHz}$. The plots are presented in figure 5.8 \& figure 5.9.

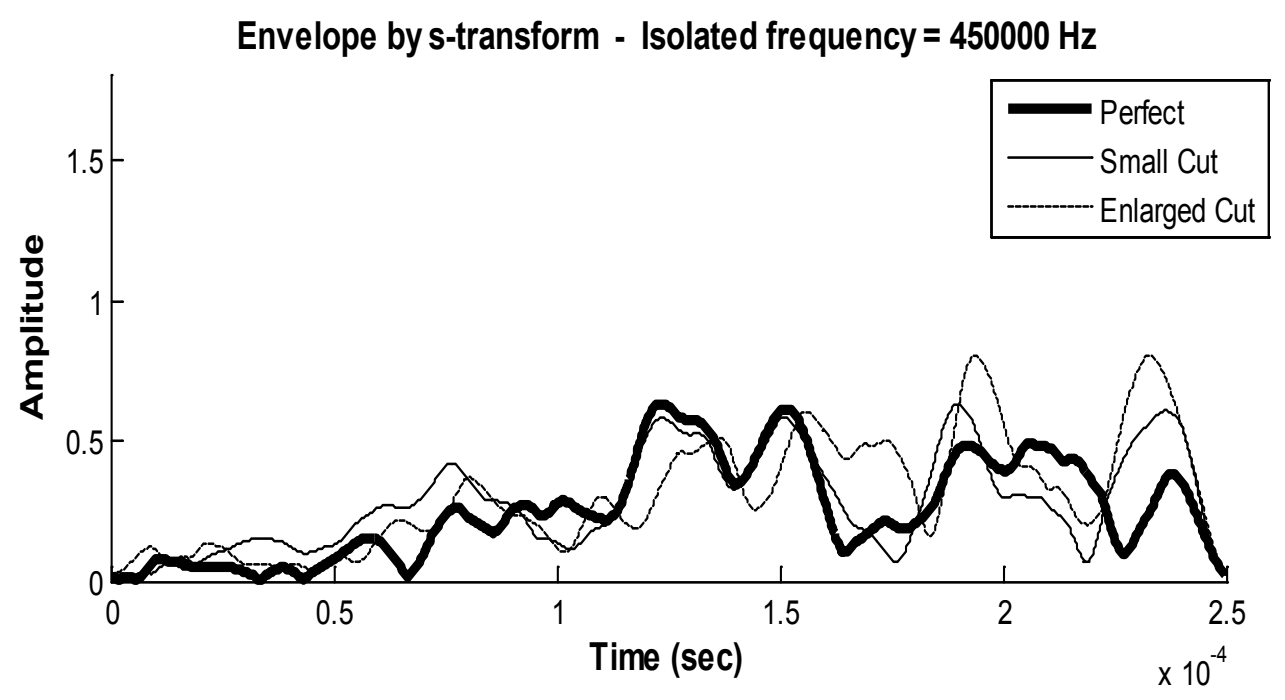

Figure 5.8 Comparison of the envelopes of the signals for three stages of defects (perfect, small cut and enlarged cut) at sensor 1 


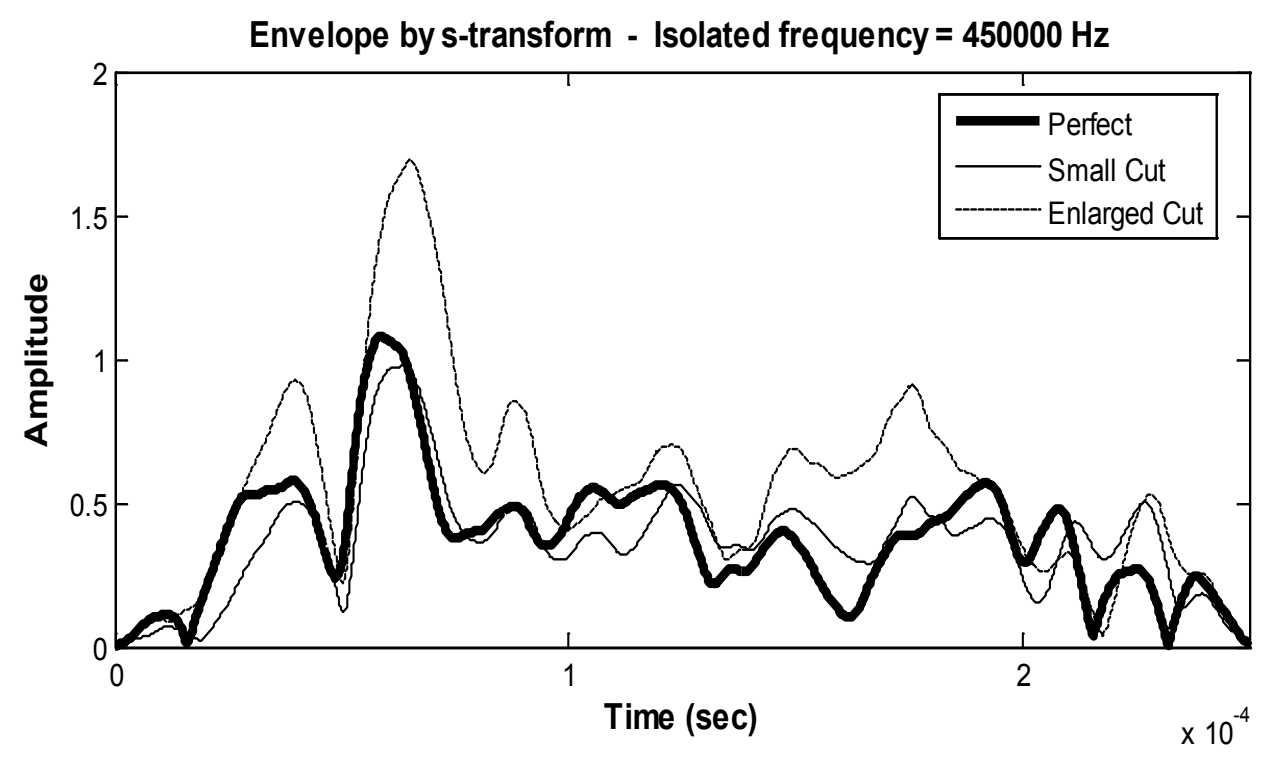

Figure 5.9 Comparison of envelopes of the signals for three stages of defects (perfect, small cut and enlarged cut) at sensor 2 .

The results obtained after generating the envelope of the signals were very promising. As seen in the above plots, the amplitude of the signal increased with the increase in the intensity of cut as demonstrated in the plot. Since the position of sensor 2 is closer to the actuator, two things are noticed in above two plots. First; the amplitude of the signals at sensor 2 is higher as compared to sensor 1 and second; the change in the behavior of signals at sensor 2 occurred a little early than compared to sensor 1 . So, it can be said that the position of the sensors on the structure has to be considered in these type of monitoring techniques.

Same experiments were performed for different type of defect (hole) and their response was analyzed. Three holes of 0.0625 inch diameter were drilled into the specimen at three locations; center, left side of weld 
and right side of the weld and the response of the signal was collected from both the sensors $(1 \& 2)$. As discussed in previous case, the experimental signal response for different location of the holes comes out to be different from each other, but nothing can be concluded or distinguished from these results (figure 5.10 \& figure 5.11).

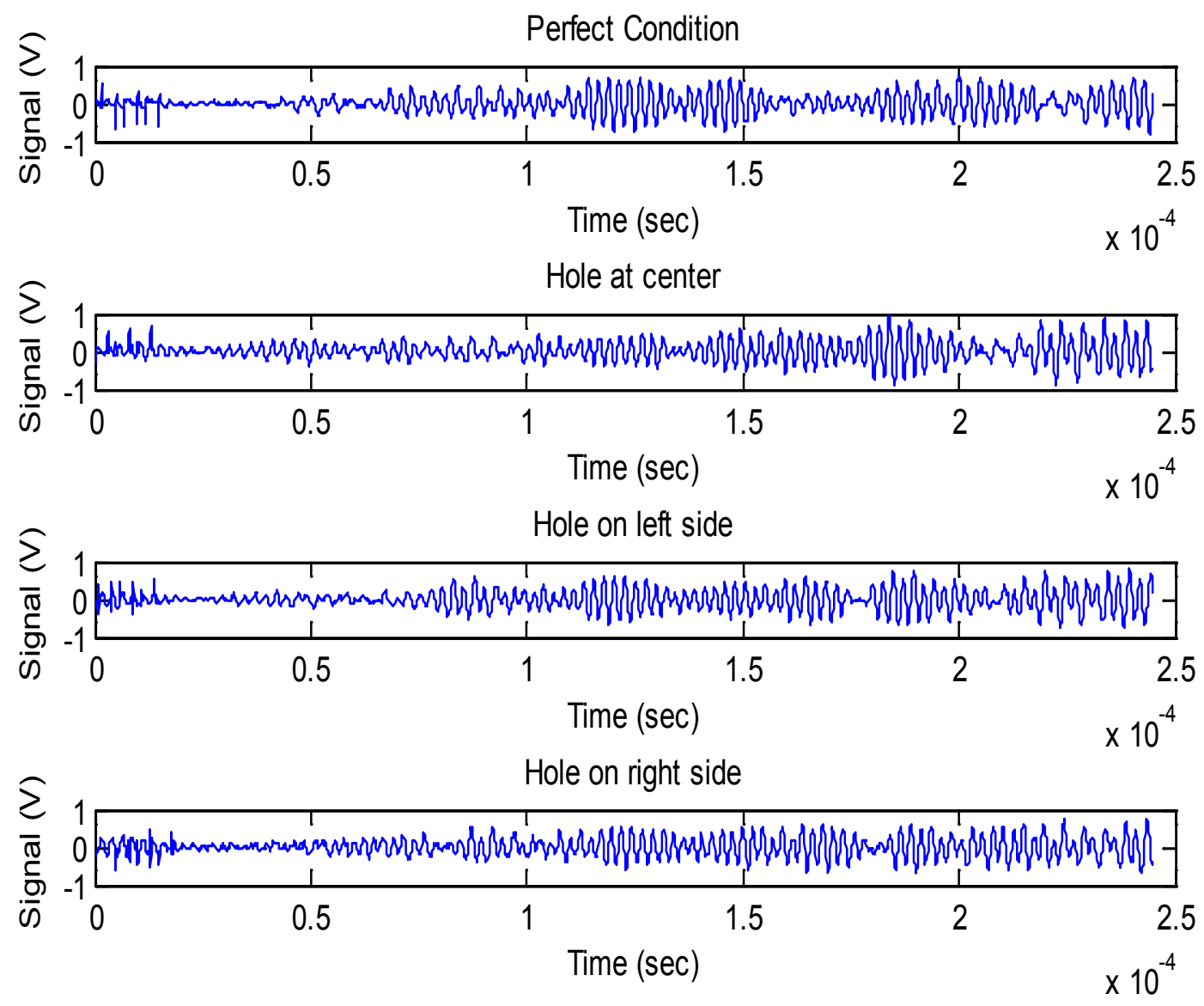

Figure 5.10Lamb wave response for perfect condition and three hole positions at sensor 1 


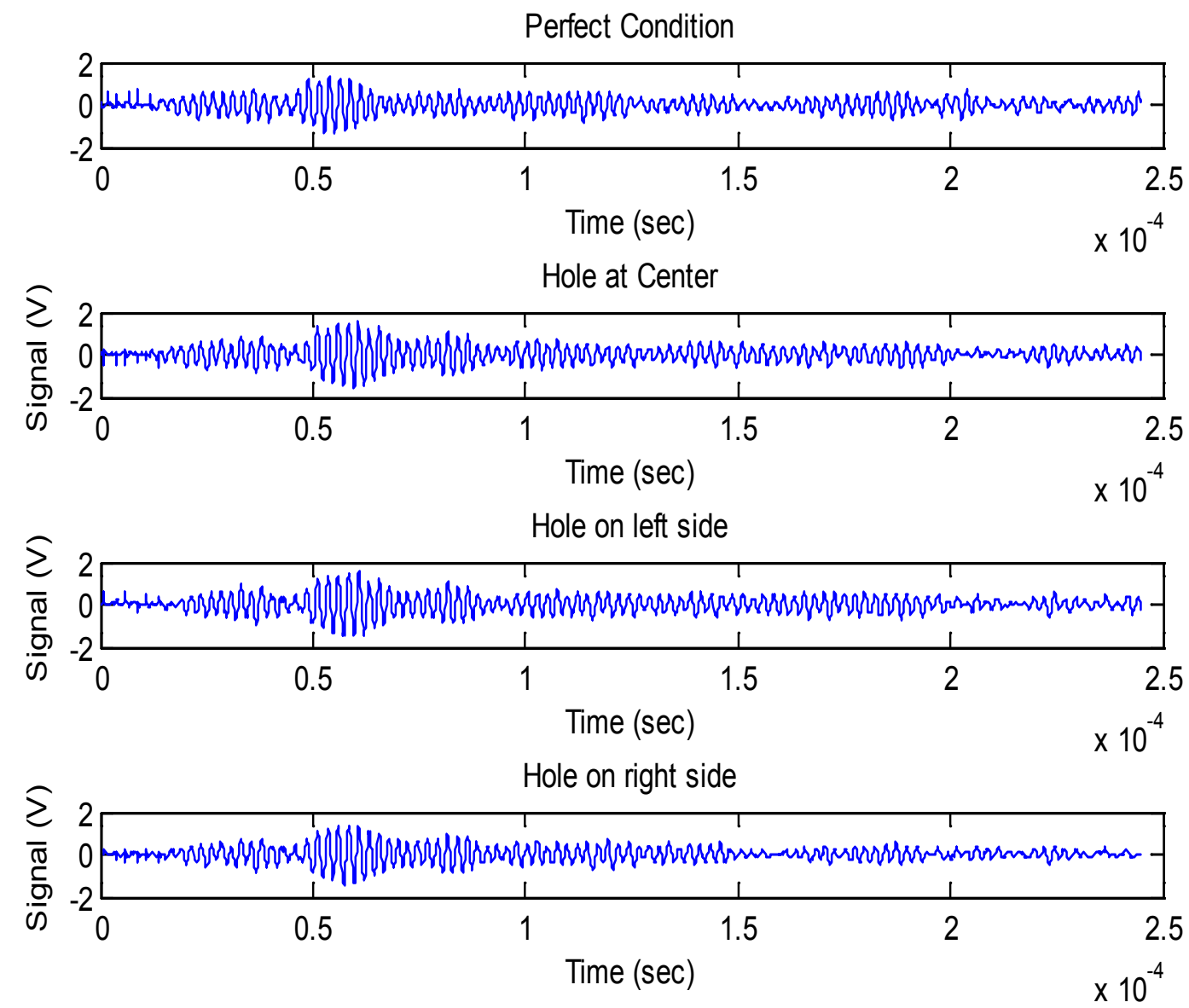

Figure5.11 Lamb wave response for perfect condition and three hole positions at sensor 1

To evaluate the signal with more details and to obtain the range of the excitation frequency, the S-transformation of the above signals was calculated and plotted in figure 5.12. 


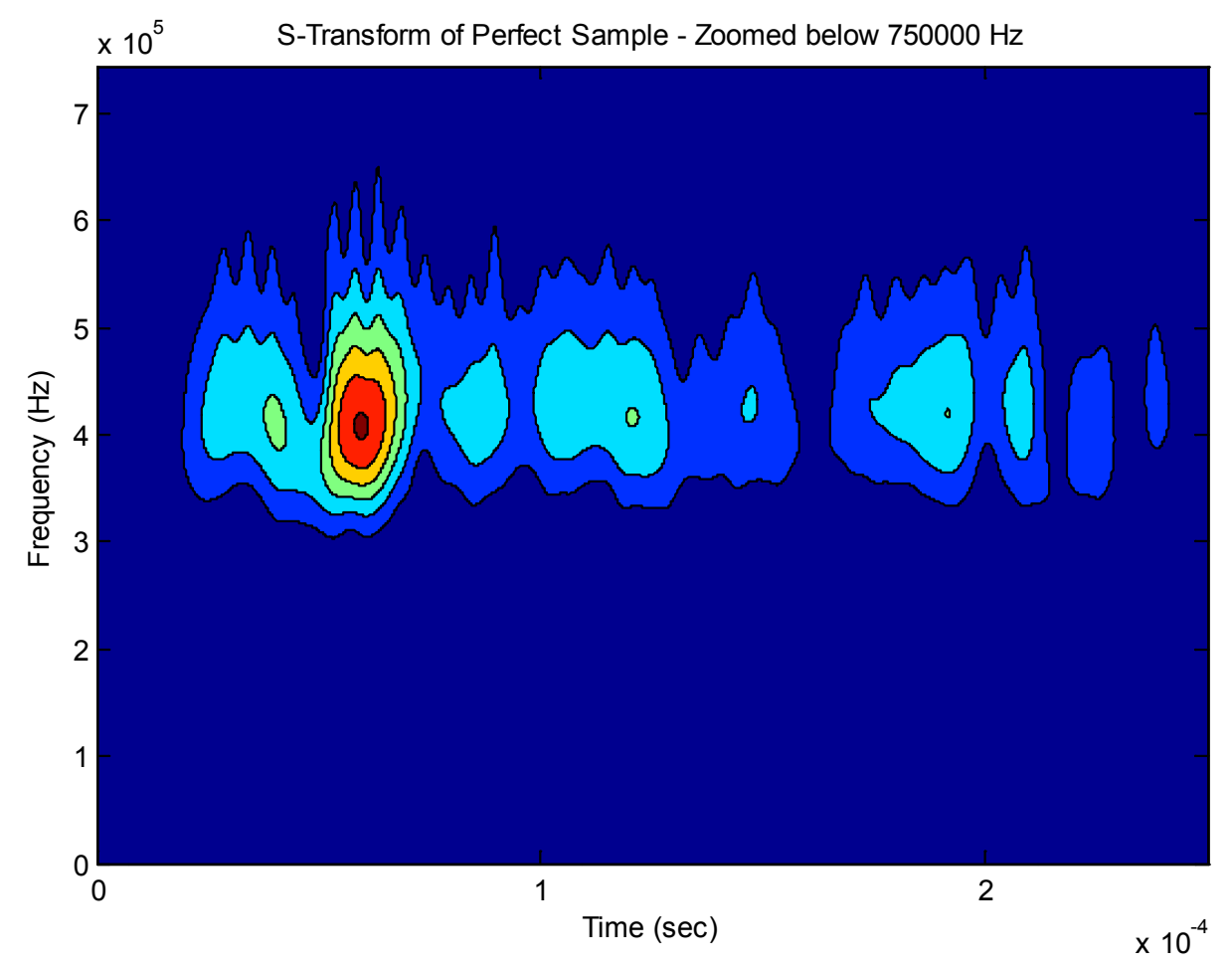

Figure5.12 S-transformation of dynamic response of perfect plate

From above figure, it can be seen that the excitation frequency came out to around $400 \mathrm{KHz}$. After the excitation frequency was determined by calculating S-transformation, the envelopes of the signals were generated(figure 5.13). The purpose of generating these envelopes was to identify the change in signal after the defects occurred in the structure. 


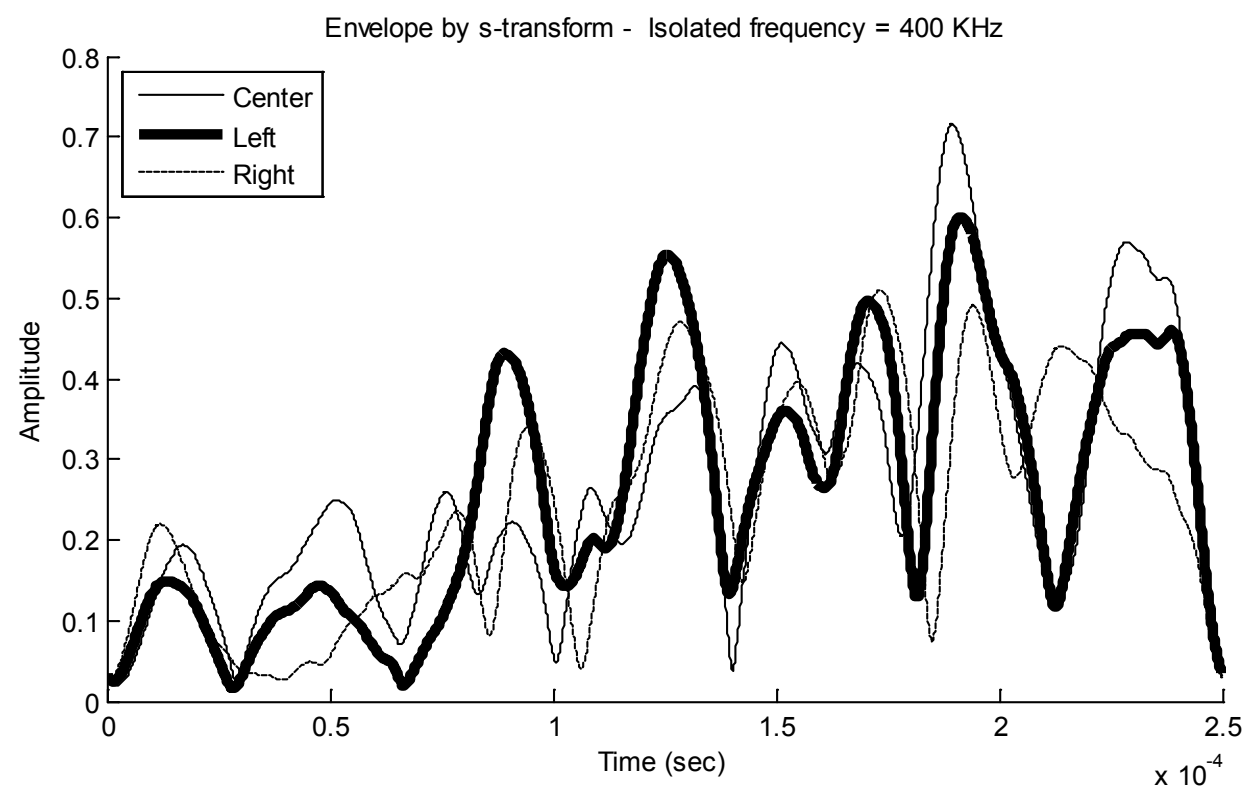

Figure 5.13 Comparison of envelopes of signals for three identical hole positions.

From the results above it can be seen that the Lamb wave approach provided promising results for defects caused by hole. The three arrows in the plot show the signal response from the defect positions. Since the size of the holes was identical, the amplitude for all the three cases was approximately the same. And regarding the positions of these peaks, the peak of left hole occurred first followed by the hole in middle and then the hole on right side of the weld because position of these holes was in the same order from the sensor as they occurred in the plot.

\subsection{Results and discussions: Surface Response to Excitation}

For the second approach, the frequency response characteristics of the (SuRE) were evaluated. One of the piezoelectric elements was excited by sweep sine wave, and the magnitude of the measured signal at other 
piezoelectric elements was calculated using a spectrum analyzer. The actuator on the specimen was excited by sweep sine wave with frequency range from 1- $100 \mathrm{KHz}$ and recording up to 2000 data points in each case. The waves thus generated by the actuator travelled through the specimen and their response was recorded by the sensors bonded onto the surface of the specimen. First, the analysis of the cut/crack was done. The measured frequency response characteristics at different crack lengths are plotted in figure 5.14.
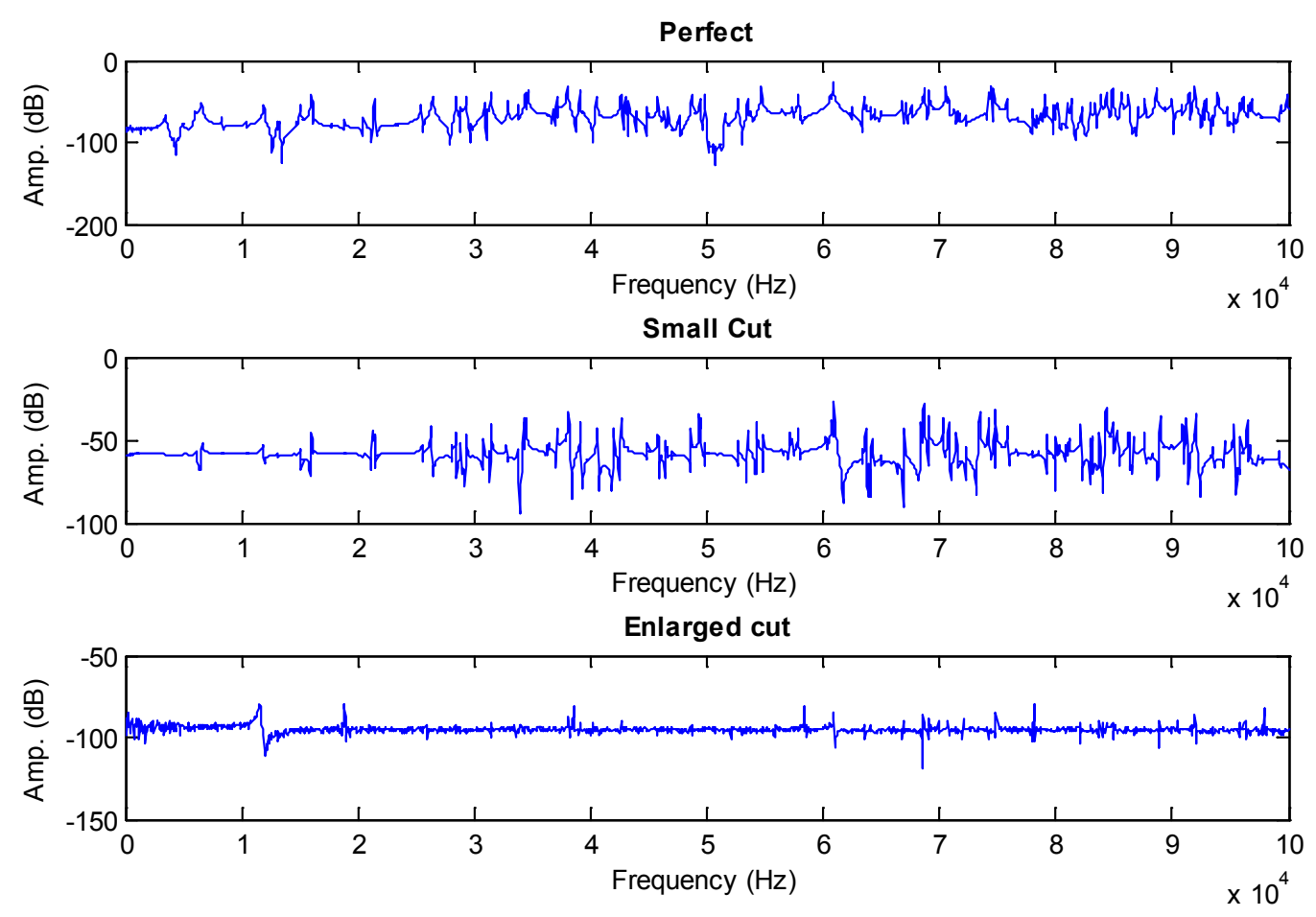

Figure 5.14 Sweep sine wave responses

From the measured frequency response obtained above it can be seen that the characteristics of the response of the signal are very different from each other, but it can also be seen that it is very difficult to distinguish these plots on the basis of the defect size. Hence to understand and categorized the signal response from each other, the sum of the 
squares of the difference is calculated at each frequency (figure 5.15). The data from the perfect condition was taken as the base line data and the square of difference of two test cases was subtracted from it.

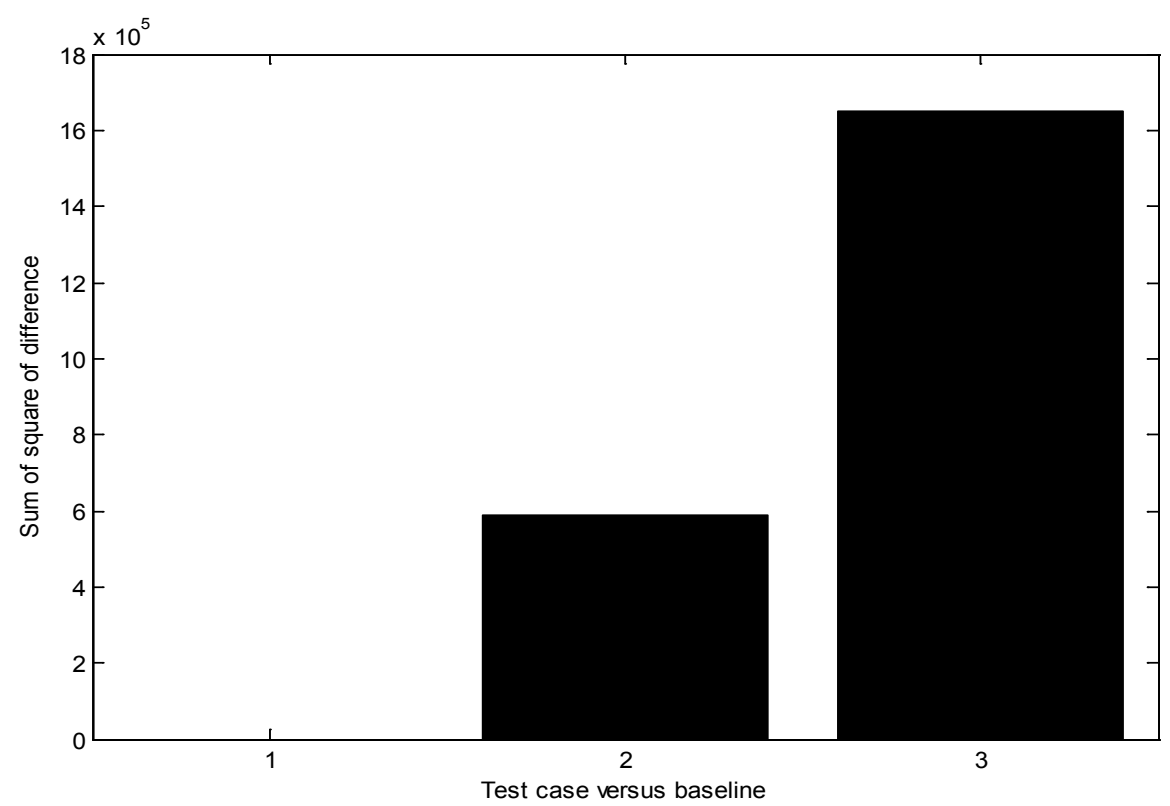

Figure 5.15 Sum of square of difference between defected and perfect sample

It can be seen clearly from the above result that the sum of the squares of the difference of the frequency response characteristics indicated the severity of the defect (cut). Data 2 and 3 in plot represents the small cut and enlarged cut respectively whereas 1 represents the baseline data. To calculate the most significant frequency range between 1-100 KHz, sum of square of the difference was generated after every 25 $\mathrm{KHz}$ frequency interval and the results were compared (figure 5.16). 


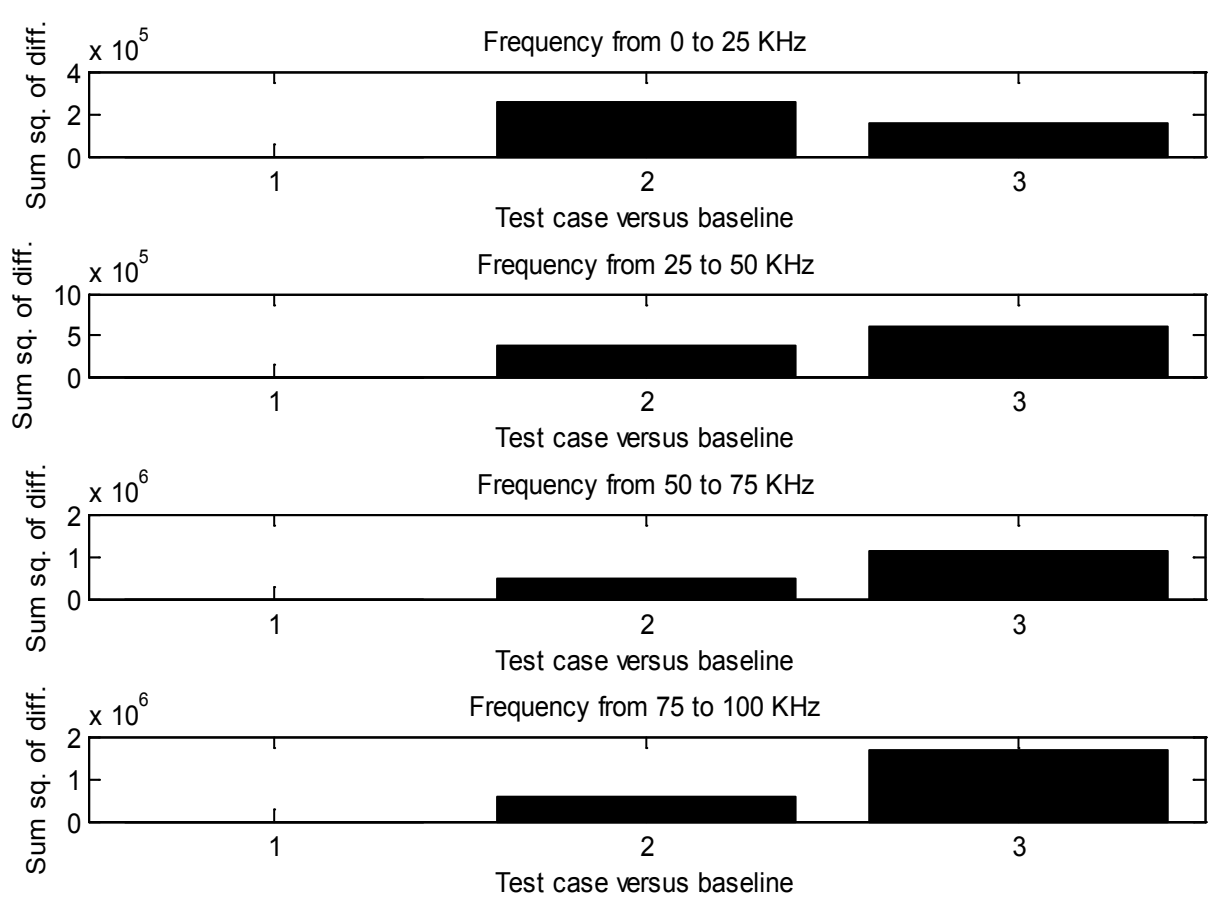

(a)

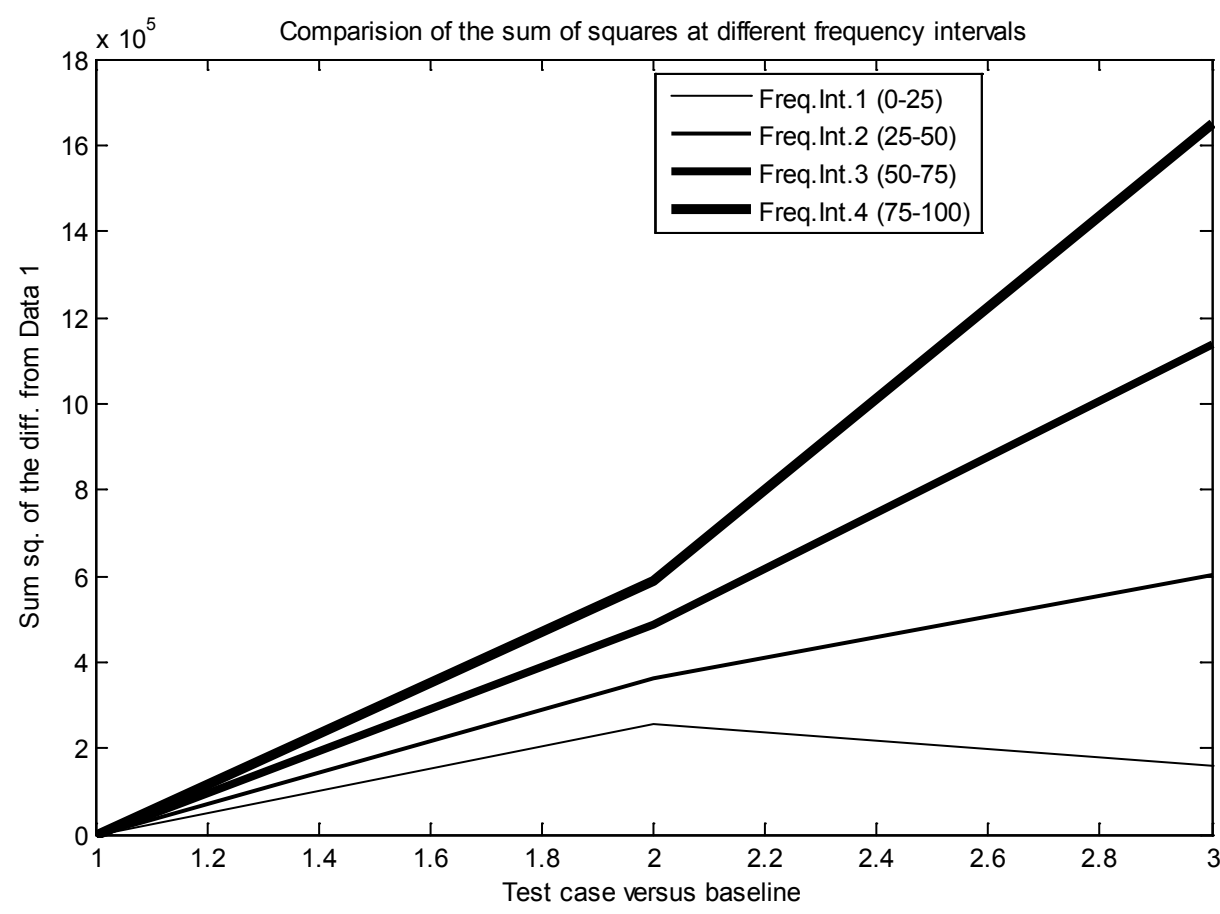

(b)

Figure 5.16 Sum of square of difference at four different frequency intervals (a) Bar graph representation (b) Linear representation

Since the maximum difference between the small cut and the enlarged cut was illustrated in the frequency interval 4 , hence from the 
results above, then most significant frequency range for this test case is from 75-100 KHz. The results were demonstrated in two different ways; bar graph and linear representation. The linear representation of the results is more understandable as compared to the bar graph representation.

After the defect due to a cut was distinguished and characterized, the same analysis was performed on a different type of defect i.e. a hole. The measures frequency response was first recorded by exciting the actuator with a sweep sine wave with a frequency range from $1-100 \mathrm{KHz}$ and the output wave was collected from the sensor bonded onto the surface of specimen using a spectrum analyzed. Since all the three holes were identical to each other, the measured frequency response was almost the same for all three cases as shown in figure 5.17. 

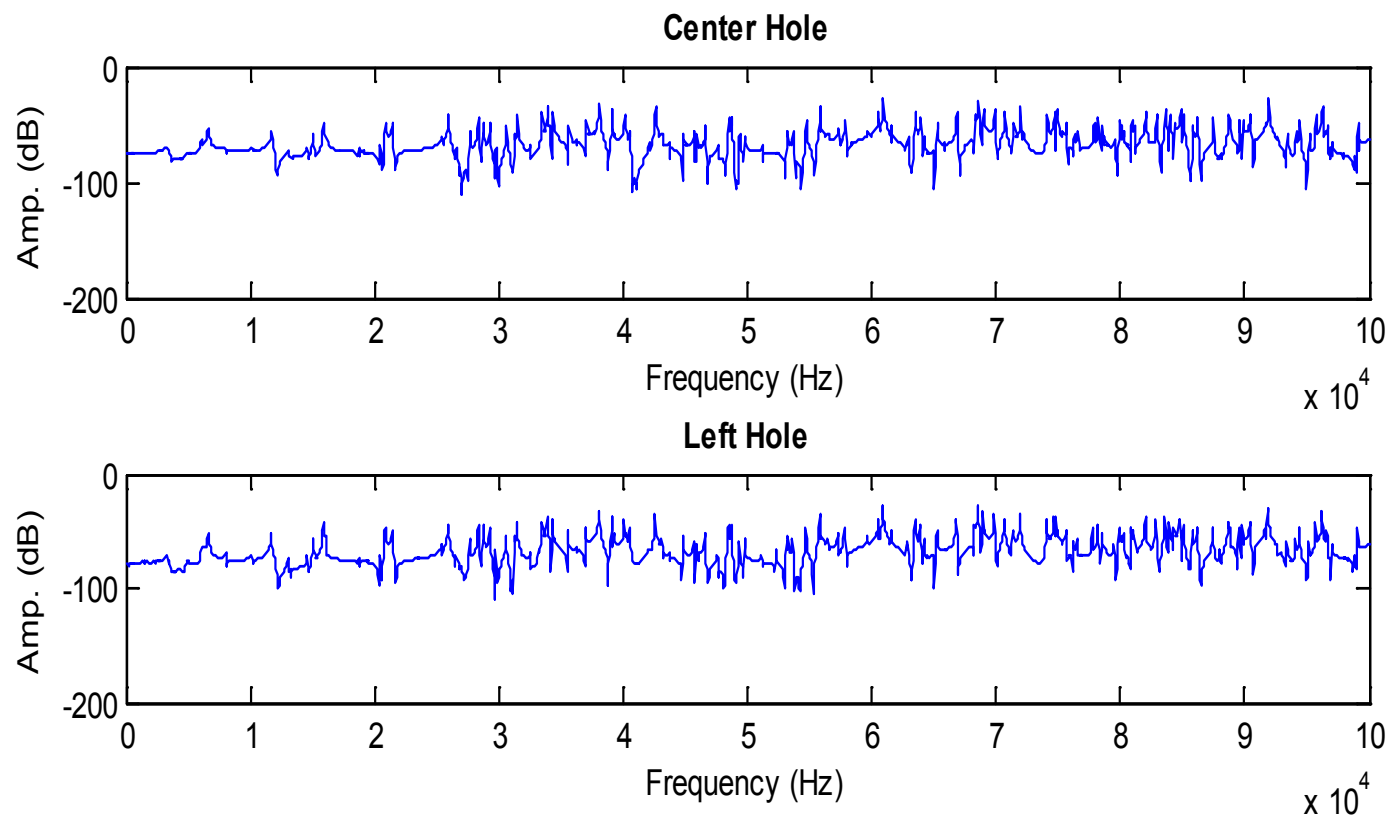

Right Hole

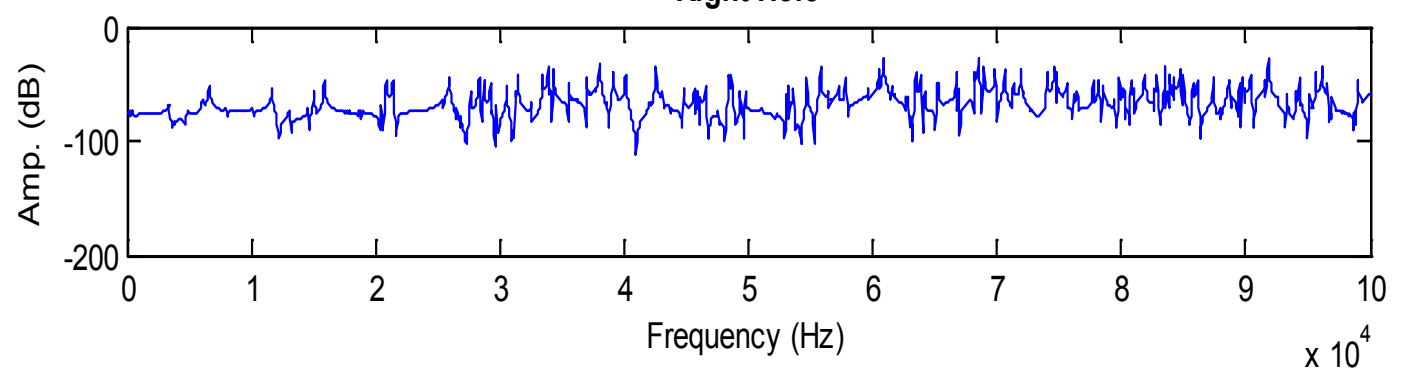

Figure 5.17 Sweep sine wave response for three hole positions

After the frequency response, the sum of square of the difference was calculated versus the baseline data. Since the three holes made in the sample were identical to each other, the magnitude of the square of the difference for all the three cases was almost same as shown in figure 5.18. As seen in figure below, the bar graph 2, 3 and 4 represents the three holes and 1 represents the baseline data. 


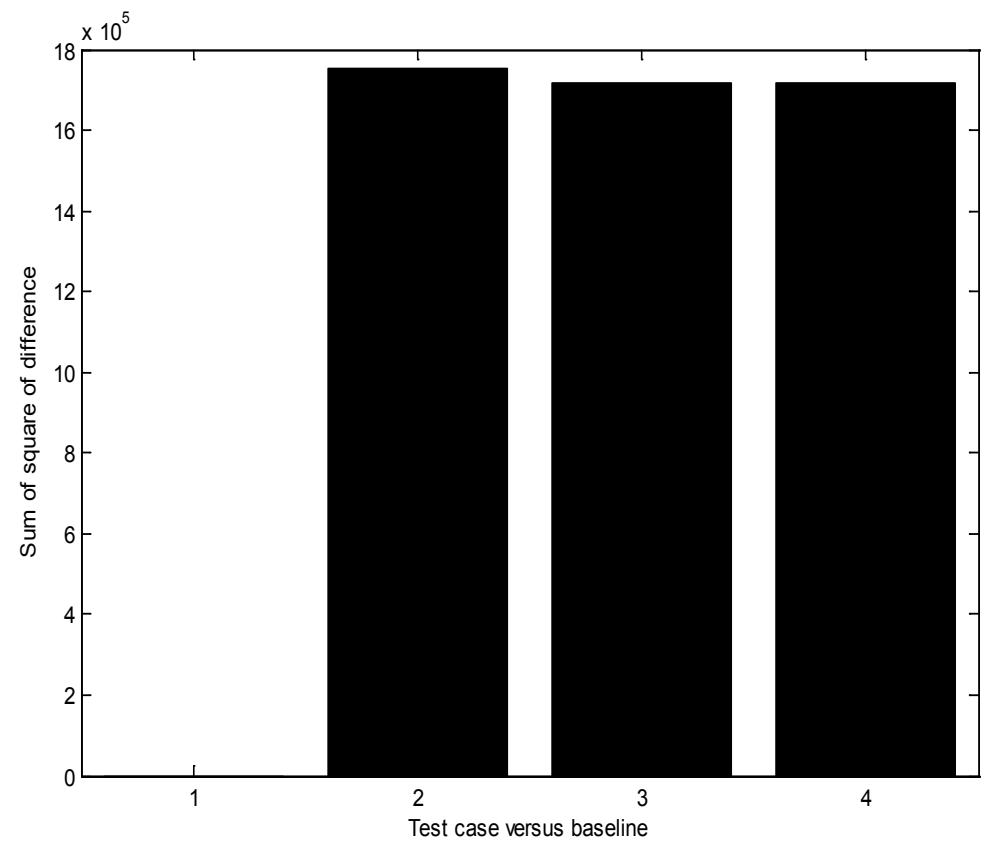

Figure 5.18 Sum of square of difference between perfect and three identical holes signals
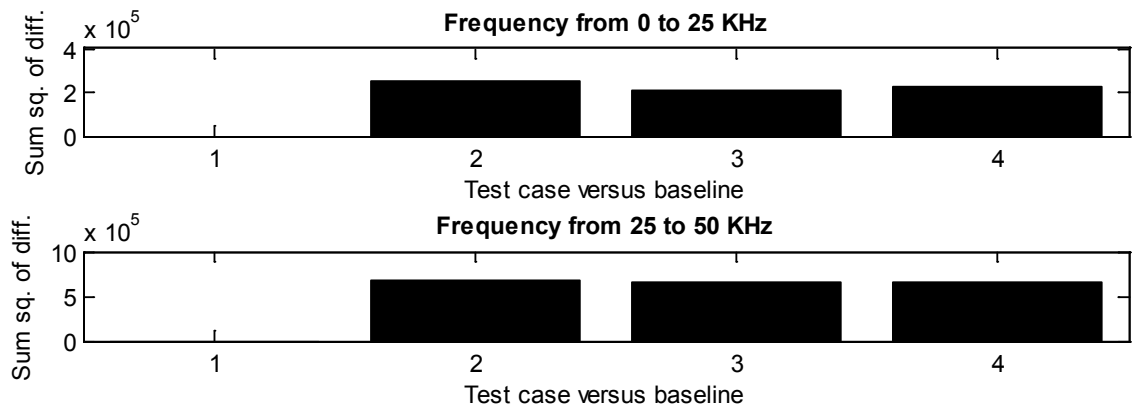

Test case versus baseline

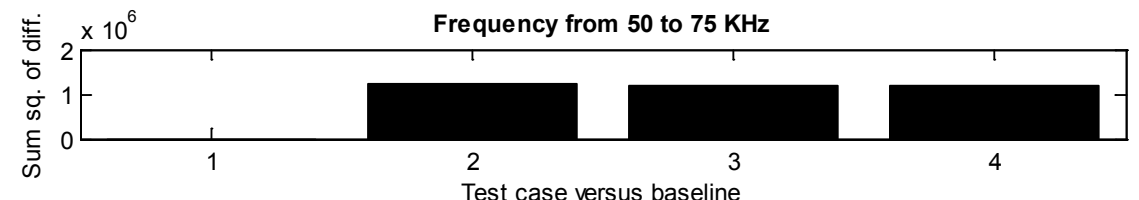

Test case versus baseline

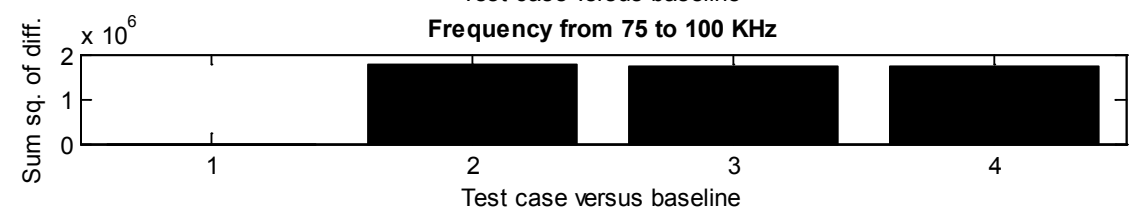

(a) 


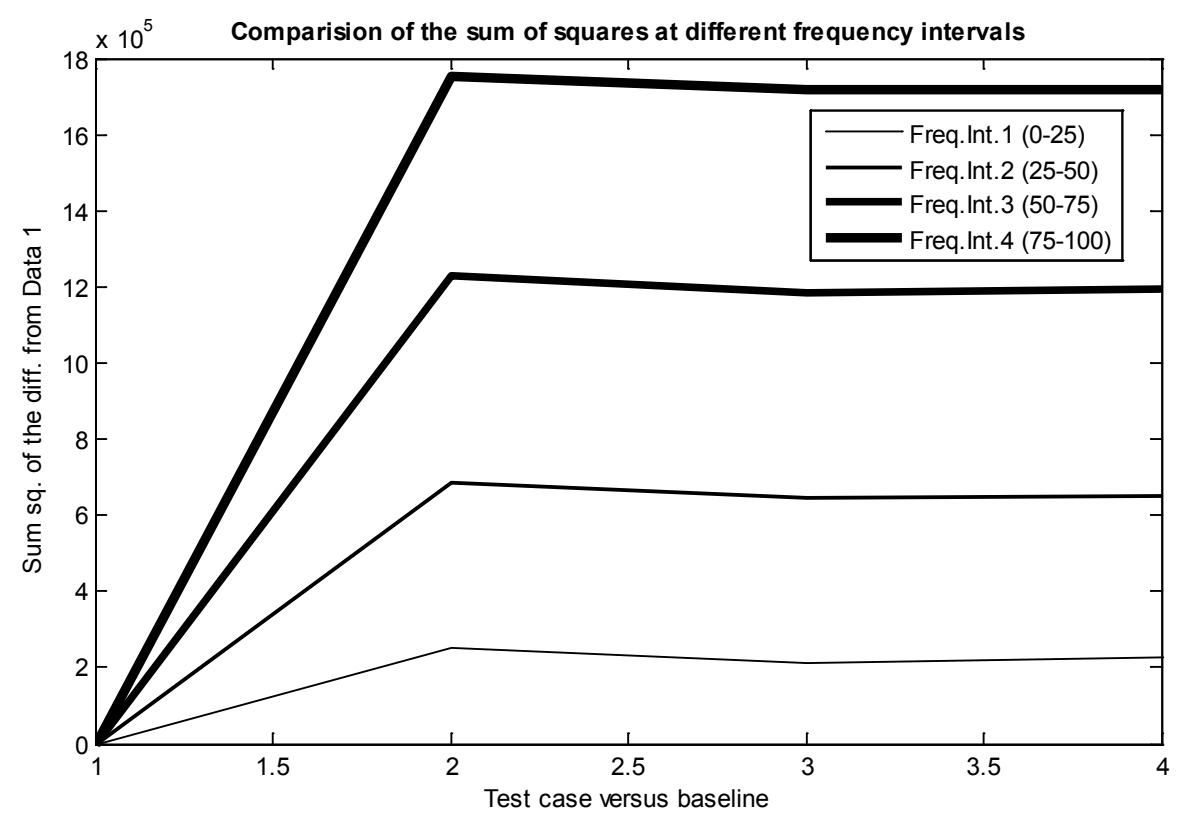

(b)

Figure 5.19 Comparison of sum of squares at different frequency intervals (a) Bar graph representation (b) Linear representation

After the square of difference was calculated, the total frequency range was divided into four intervals, in order to calculate the most significant interval. The purpose of doing this was to find a frequency range that demonstrates the maximum difference between the perfect and defected condition. From figure 5.19, the frequency range from 75-100 $\mathrm{KHz}$ was the most significant range. The surface response to excitation approach proved to be an ideal approach for determining the intensity of the defect.

\subsection{Analysis validation and benefits}

The repeatability and consistency of the experimental procedure and analysis approach is an important factor to be considered in any research being held these days. So, in order to validate the procedure and analysis 
that were done in order to obtain above results, all the above experiments were performed couple of times keeping all conditions identical. Due to large number experiments and enormous data sets, one of the experiments was selected to validate the system. The data from the welded plate defects was selected as a test case. First the envelopes of the propagated signals were generated and compared in the figure 5.20. It can be clearly seen that data $1 \&$ data 2 in the plot below are almost identical to each other and similarly data 3 and data 4 are very close to each other, yet there was a little bit difference between the two identical signals.

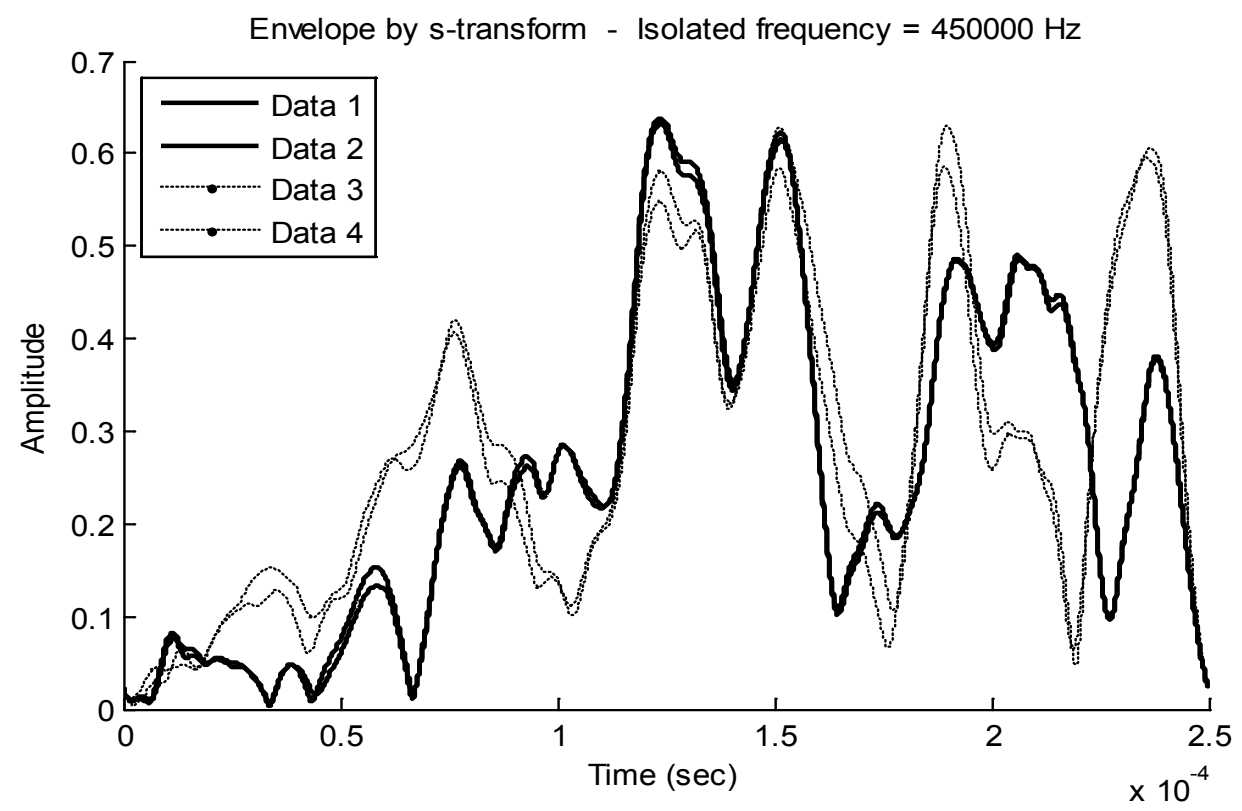

Figure 5.20 Comparison of envelopes of two identical data sets.

So, in order to calculate the magnitude of difference between two identical data points, the absolute difference between these data points was calculated by comparing the peaks of the signals in time domain (figure 5.21). It can be clearly seen from the figure below, that the absolute 
difference in both cases came out to be very small as compared to the original signals. Hence, the repeatability of the monitoring technique including the experimental procedure is validated.

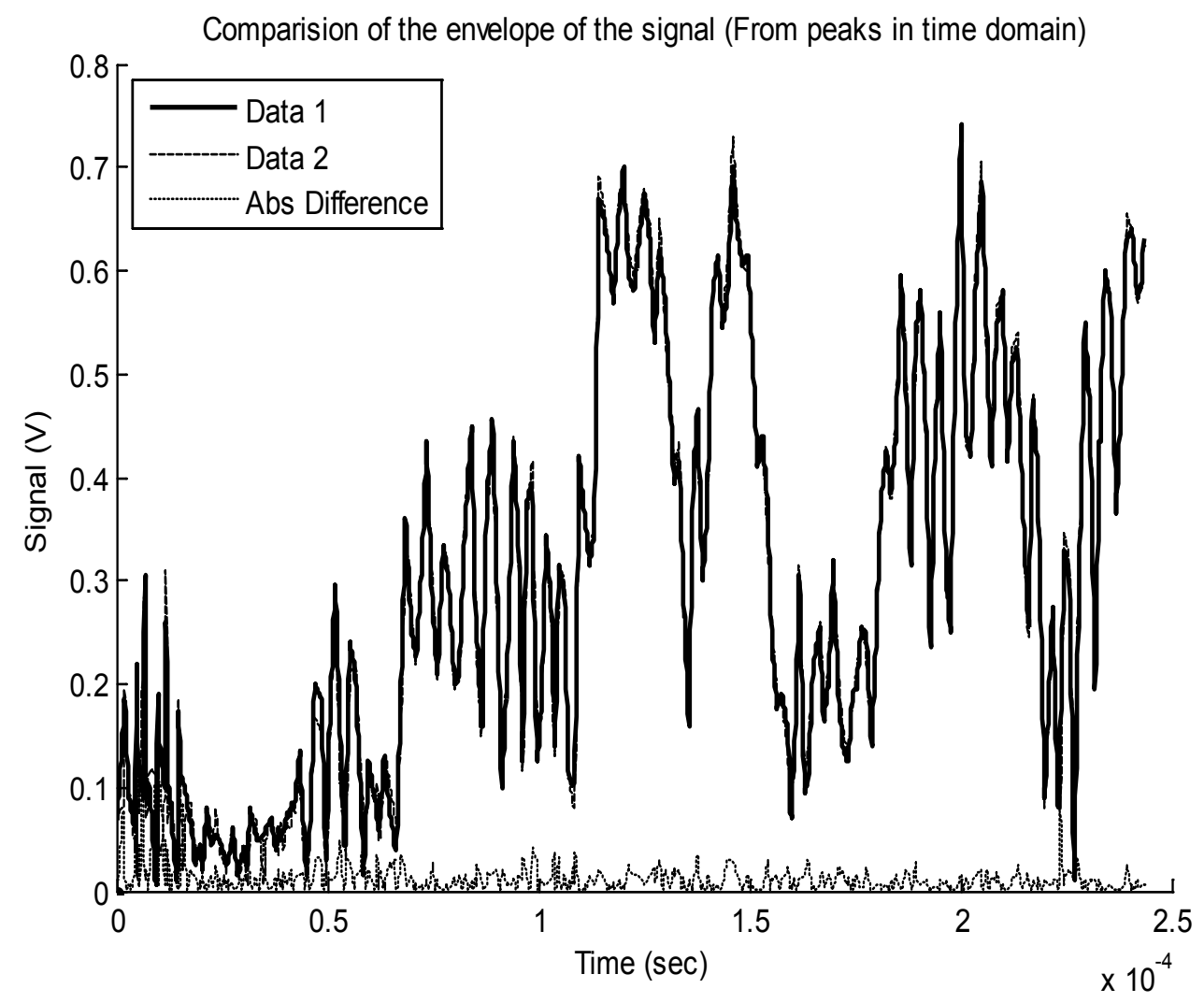

(a) 


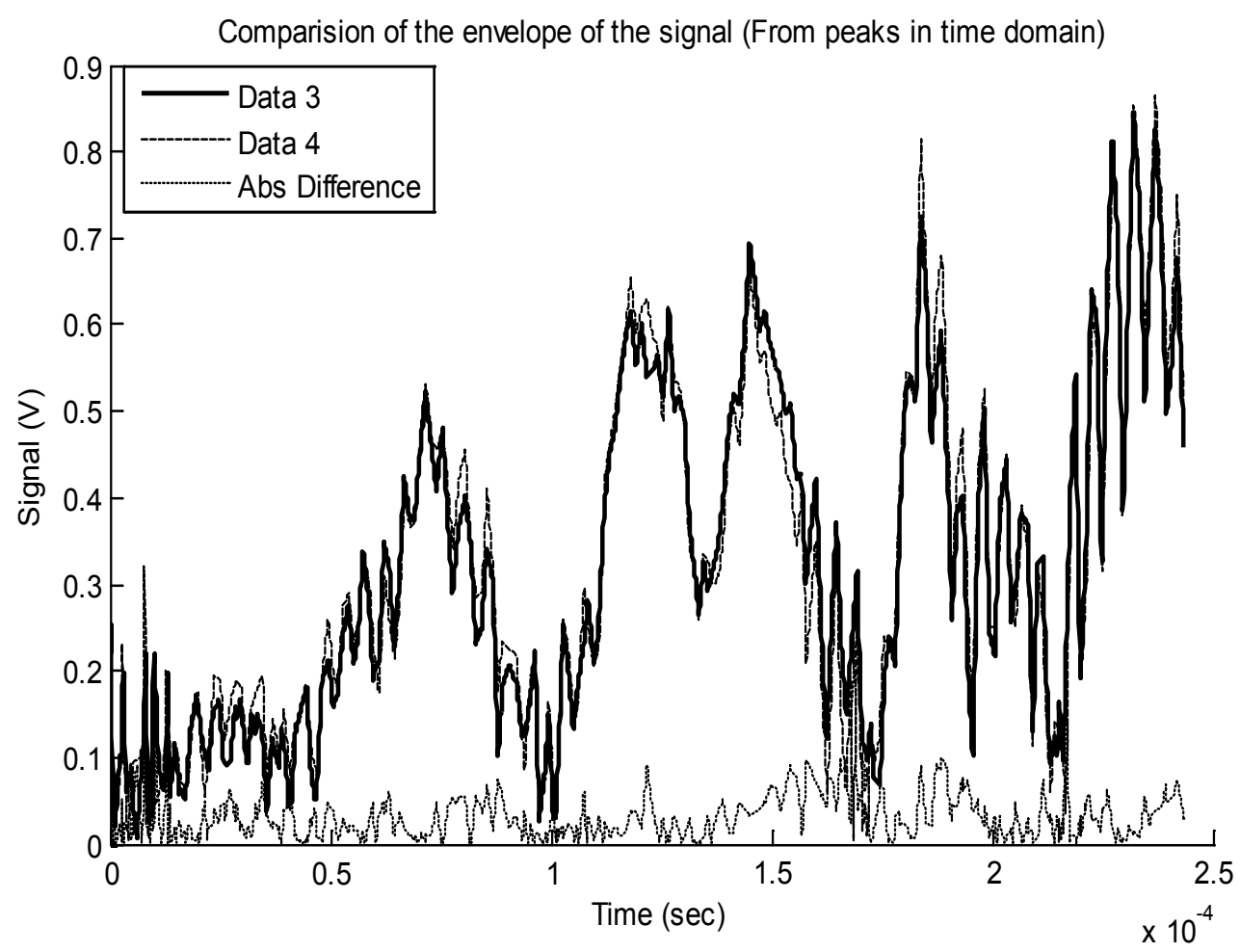

(b)

Figure 5.21 Absolute differences between two identical sets of data in time domain (a) Difference between identical data $1 \& 2$ (b) Difference between identical data $3 \& 4$

Since a large amount of data points has to be collected in order to obtain good results for the Lamb wave analysis, it becomes very cumbersome to analyze the data. Hence, the wavelet transformation of the original data was done to compress the data. Wavelet transformation is a mathematical tool that is quite useful for analyzing many types of signals. It has been proven especially useful in data compression. An important property of wavelet analysis is perfect reconstruction, which is the process of reassembling a decomposed signal or image into its original form without loss of information. A data at random was selected and wavelet transformation of that data was calculated as shown in figure 5.22. 
1 - level WT - The envelope of the original signal - Data points 1
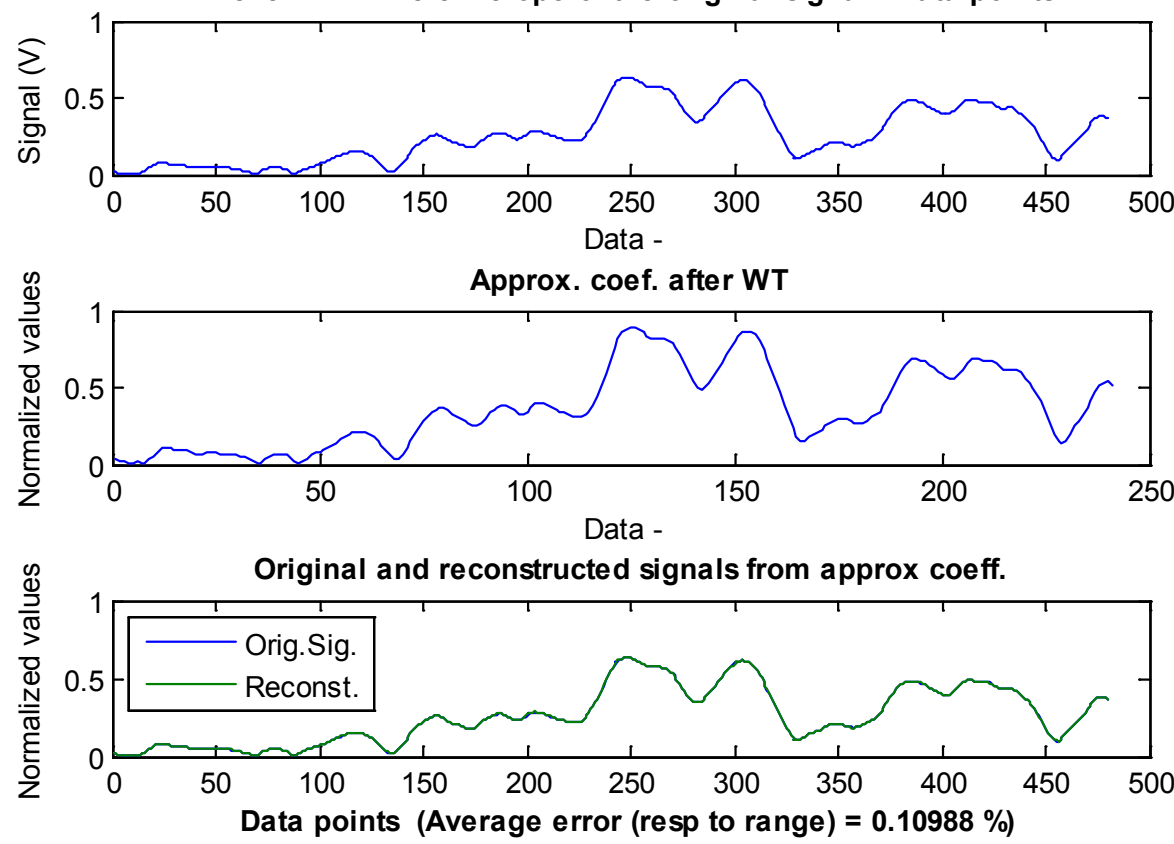

(a)
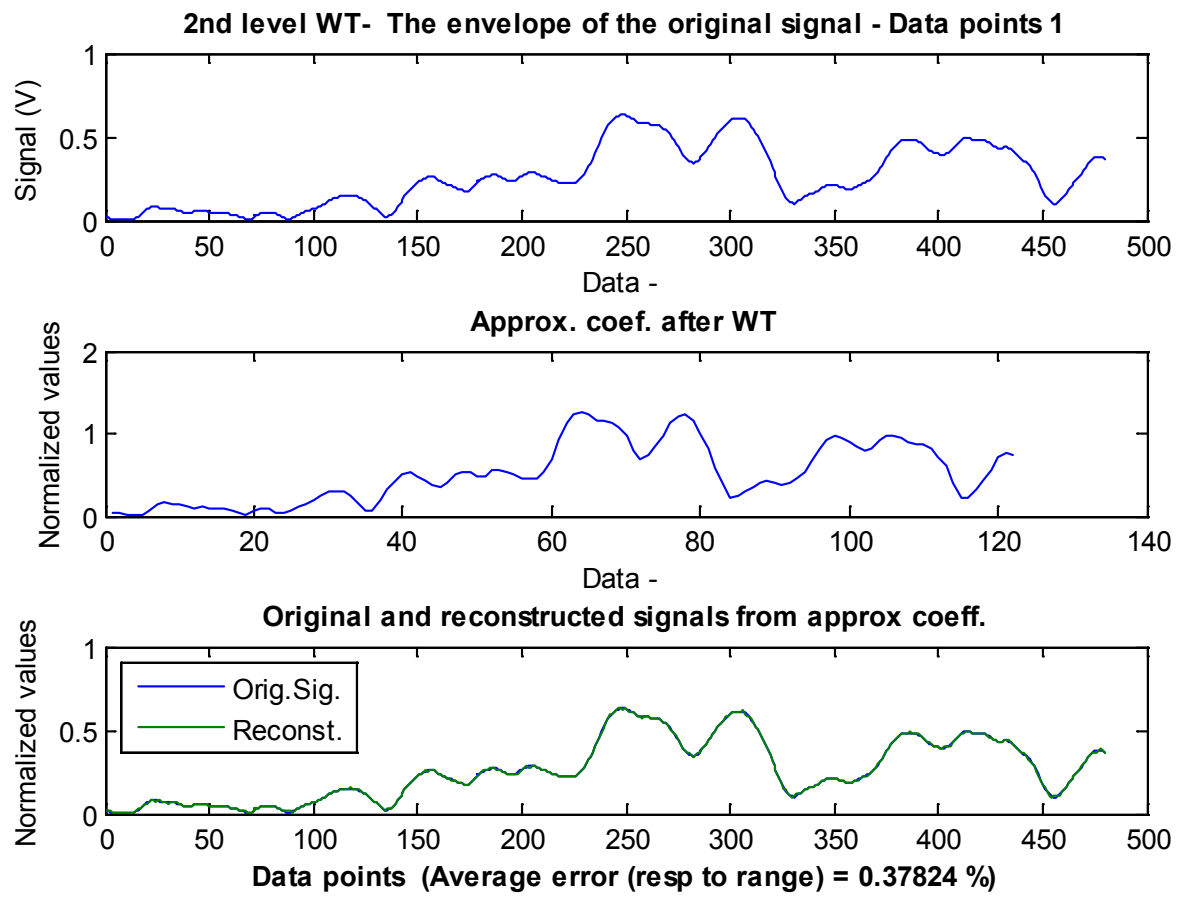

(b) 

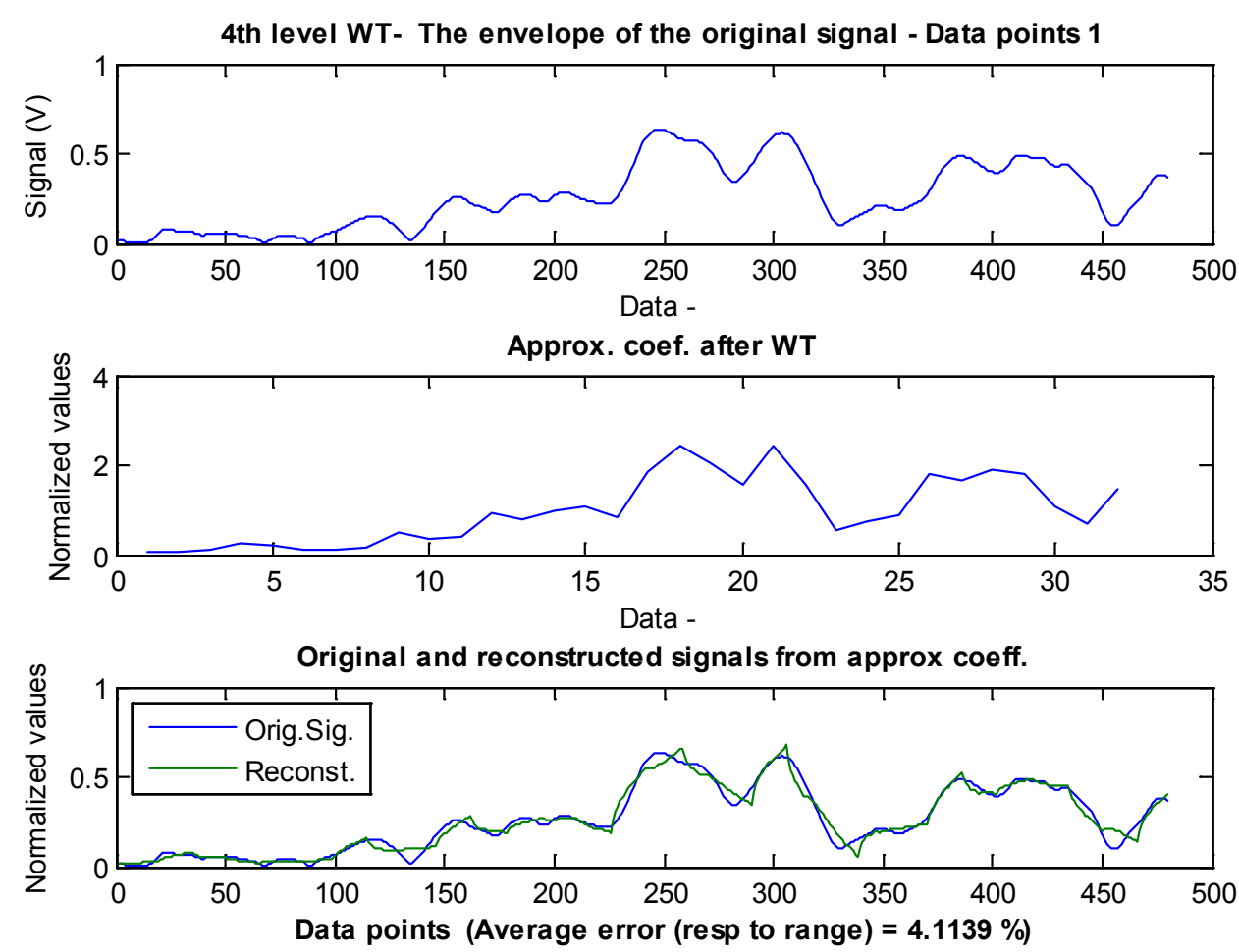

(c)

Figure 5.22 Wavelet transformations of random data (a) First level (b) Second level (c) Fourth level

A total of 4 levels of wavelet transformations were done and the results are plotted above. After the first level of wavelet transformation, it can be seen that there was $50 \%$ reduction in the total number of data points, whereas the average error between the original signal and approximate coefficient was only $0.1 \%$. As the levels kept on increasing, the error percentage also increased. We can see that at $4^{\text {th }}$ level, there was a $93 \%$ decrease in number of data points and the error was raised to $4 \%$. For an enormous amount of data, that requires much advanced equipment's can be compressed to a certain number by taking the absolute error into consideration. For life sustaining structures such as bridges, 
aircrafts, etc. or the structures operating in extreme conditions such as dams, nuclear plants etc. the error percentage should be minimum as compared to other structures in order to detect any defect before sudden failure of the structure. Hence wavelet transformation is proved to be a good tool for Lamb wave data analysis where a large amount of data can be represented by a comparatively small amount of the data points without any/much loss of important information.

\subsection{Conclusion}

Both approaches Lamb wave propagation and surface response to excitation were evaluated to monitor the defects in a welded plate structure. In the Lamb wave approach, the signals of interest were isolated by using the S-transformation and then the envelopes of those signals were compared, whereas the surface response to excitation (SuRE) method calculated the surface response characteristics and evaluated the intensity of defect.

The results from both approaches were complementary to each other, as the envelopes of the generated Lamb wave signals changed as the defects keep on changing and similarly the frequency response for the SuRE method also showed a significant change due to defects. Hence both approaches could be used for monitoring the structure with a welded joint. In Lamb wave approach, the envelopes indicated the position of the defects, but were not able to indicate clearly the severity of the defects where as an opposite condition was seen in SuRE approach. The sum of 
squares of the difference of the frequency response characteristics indicated the severity of the defects and can be used for any condition irrespective of the size and shape of structure. Both approaches are in expansible due to the low cost of the sensors and equipment's and over that, the SuRE approach was similar to the impedance method but the overall cost and complexity of the equipment is much less. 


\section{CONCLUSION \& FUTURE WORK}

\subsection{Conclusion}

Structural Health Monitoring is an area of growing interest. SHM using embedded PWAS for the detection of material damages with Lamb wave propagation methods, which are now being profoundly researched as having the most potential for damage detection in plates and SuRE method, which have traditionally received the most attention. Detection and monitoring of defects in plates has been an interesting area of scientific study in the past two decades. Particularly, the dynamic characteristics of a crack in a plate/plate like structures and their monitoring have received considerable attention in this area of research.

This thesis starts from exploring the durability and sustainability of the sensory system including the adhesive materials under different atmospheric conditions. Several experiments were performed using various combinations of the sensors and bonding materials. The sweep sine wave approach was used to generate the surface waves, and the best combination for each condition was concluded on the basis of weekly response of the system.

An extensive amount of experimental data for the healthy, 30\% cracked and 50\% cracked plate sample has been collected, analyzed and presented. The obtained results demonstrate that the external excitation force with a calculated frequency range when applied on the cracked plate induces combinational frequencies. These combinational frequencies 
provide an indication of the presence of a crack. From the experimental results, it was observed that the amplitude of the frequencies amplify when the crack depth increases. Moreover the amplitude of the frequency peaks depends on the location of the sensor. In addition, the Lamb wave propagation method has been presented in detail. The results from both approaches have been compared and were complementary to each other.

In another set of experiments, the defects in the welded plate structure were monitored. Both techniques successfully detected the intensity of the damage and in addition, the Lamb wave method was able to detect the approximate location of the identical defects in the structure. It is thus concluded that both Lamb wave and SuRE methods were able to monitor and detect the damage intensity for varying size and thickness of the plate structure.

\subsection{Recommendations for Future Work}

Much work still remains before current SHM systems can be relied upon to replace standard inspection and maintenance sequence.

- For frequency response method, more experiments should be performed on built-up and larger structures to validate the initial results. An experimental study paralleling the performed tests but varying the size of the damaged regions would also be useful in determining this method's sensitivity. 
- $\quad$ For Lamb wave testing, further work lies ahead for different types of built-up structures other than the ones tested during the present research.

- Another possibility would be to test these methods on materials in use, since the present work only indicated newly manufactured specimens with artificially introduced damage, and did not considered the complexities in an aging structure.

- $\quad$ Remotely controlling and accessing data from sensors via wireless connection still needs much attention. 


\section{REFERENCES}

1. C. R. Farrar and K. Worden, "An introduction to structural health monitoring”. Phil. Trans. R. Soc. A (2007) 365, Pages 303-315.

2. S. Park, C. Yun and Y. Roh, "Damage diagnostics on a welded zone of a steel truss member using active sensing network system. NDT\&E International 40, 2007, Pages 71-76.

3. J. N. Schoess and J. D. Zook, "Test result of resonant integrates microbeam sensor (RIMS) for acoustic emission monitoring". Proceedings of the SPIE conference on smart electronics and MEMS, vol. 3328, 1998, Pages 326-332.

4. L. Kollar and R. J. Steenkiste, "Calculation of the stresses and strains in the embedded fiber optic sensors". J. Compos Mater, 1998;32, Pages 1647-1679.

5. V. Giurgiutiu, "Tuned lamb wave excitation and detection with piezoelectric wafer active sensors for structural health monitoring". Journal of intelligent material systems and structures. Vol. 16, April 2005

6. V. Giurgiutiu and C. A. Rogers, "Electro-mechanical (E/M) impedance method for structural health monitoring and nondestructive evaluation". Proceedings of international workshop on structural health monitoring. Stanford University, CA, 1997, Pages 433-440

7. G. Park, H. Sohn, C. R. Farrar and D. J. Inman, "Overview of piezoelectric impedance-based health monitoring and path forward". Shock Vibr Dig, 2003; 35(6), Pages 451-463.

8. P. Cawley and D. Alleyne, "The use of Lamb waves for the long range inspection of large structures". Ultrasonics, 1996;34, Pages 287-290.

9. V. Giurgiutiu, "Lamb wave generation with piezoelectric wafer active sensors for structural health monitoring". Proceedings of the SPIE conference on smart structures and materials, vol. 5056, 2003, Pages 111-122.

10. C. Boller and N. Meyendorf, "State of art in structural health monitoring for aeronautics". Proc. Of Internat. Symposium on NDT in aerospace, Bavaria, Germany 3-5, 2008. 
11. Z. Su, L. Ye and Y. Lu, "Guided lamb waves for identification of damage in composite structures: A review". Journal of sound and vibration 295, 2006, Pages 753-780.

12. M. J. S. Lowe, "Matrix techniques for modeling ultrasonic waves in multilayered media". IEEE transactions on ultrasonic, ferroelectrics and frequency control 42 (2), 1995, Pages 525-542.

13. D. E. Chimenti, "Guided waves in plates and their use in materials characterization". Applied mechanics review 50 (5), 1997, Pages 247-284.

14. D.C. Worlton, "Experimental conformation of Lamb waves in megacycle frequencies". Journal of applied physics 32, 1961, Pages 967-971.

15. J. L. Rose, "Avision of ultrasonic guided wave inspection potential". Proceedings of the seventh ASME NDE tropical conference, NDEvol. 20, 2001, Pages 1-5.

16. J. D. Achenbach, "Wave propagation in elastic solids". NorthHolland publishing company, Amsterdam, 1973.

17. V. Giurgiutiu, J. Bao, "Embedded ultrasonic structural radar for in situ structural health monitoring of thin wall structures". Structural health monitoring 3 (2), 2004, Pages 121-140.

18. V. Giurgiutiu, "Structural health monitoring with piezoelectric wafer active sensors". Proceedings of the $16^{\text {th }}$ international conference of adaptive structures and technologies ICAST, 2005, Paris, France.

19. K. Hongjoon, J. Kyungyoung, S. Minjea, K. Jaeyeol, "A non-contact NDE method using a laser-generated focused-Lamb wave with enhanced defect-detection ability and spatial resolution". NDT\&E international 39, 2006, Pages312-319.

20. K. Hongjoon, J. Kyungyoung, S. Minjea, K. Jaeyeol, "Application of the laser generated focused-Lamb wave for non-contact imaging of defects in plates, Ultrasonics 44, 2006, Pages 1265-1268.

21. S. G. Pierce, B. Culshaw, G. Manson, K. Worden, W. J. Staszewski, "The application of ultrasonic Lamb wave techniques to the evaluation of advanced composite structures". Smart structures and materials-Proceedings of SPIE 3986, 2000, Pages 93-103. 
22. C. Wang, T. Rosej, F. K. Chang, "A synthetic time reversal imaging method for structural health monitoring". Smart materials and structures 13, 2004, Pages 415-423.

23. V. Giurgiutiu and A. Cuc, "Embedded non-destructive evaluation for structural health monitoring, damage detection and failure prevention". The shock and vibration digest, vol. 37, No.2, March 2005, Pages 83-105.

24. C. Stolz and M. Neumair' "Structural health monitoring, in service experience, benefits and way ahead". Journal of intelligent material systems and structures 2010 Pages 209-217.

25. S. Bhalla and C. K. Soh, "Electromechanical impedance modeling for adhesively bonded piezo-transducer". Journal of intelligent material system and structures, vol. 15, 2004, Pages 955-972.

26. F. Hey, S. Bhalla and C. K. Soh, "Optimized parallel interrogation and protection of piezo-transducers in electromechanical impedance technique". Journal of intelligent material system and structures, vol. 17, 2006, Pages 457-468.

27. F. P. Sun, Z. Chaudhry, C. A. Rogers, M. Majmundar and C. Liang, "Automated real-time structural health monitoring via signature pattern recognition". Proceedings of SPIE conference on smart structures and materials, Vol. 2443, 1995, Pages 236-247.

28. J. W. Ayres, F. Lalande, Z. Chaudhry and C. A. Rogers, "Qualitative impedance based health monitoring of civil infrastructures". Smart materials and structures, 7(5), Pages 599-605.

29. V. Giurgiutiu and A. N. Zagrai, "Characterization of piezoelectric wafer active sensors". Journal of intelligent material systems and structures, 11(12), 2000, Pages 959-976.

30. G. Park, H. H. Cudney and D. J. Inman, "Impedance based health monitoring of civil structural components". Journal od infrastructure system, ASCE, 6(4), 2000, Pages 153-160.

31. J. Sirohi and I. Chopra, "Fundamental understanding of piezoelectric strain sensor". Journal of intelligent material system and structures, 11(4), 2000, Pages 247-257.

32. V. Giurgiutiu and A. N. Zagrai, "Embedded self-sensing piezoelectric active sensors for on line structural identification". 
Journal of vibration and acoustics, ASME, 124(1), 2002, Pages 116125.

33. G. Park, H. H. Cudney and D. J. Inman, "Feasibility of using impedance based damage assessment for pipeline structure". Earthquake engineering and structural dynamics, 30(10), 2001, Pages 1463-1474.

34. S. Bhalla and C. K. Soh, "Structural impedance based damage diagnosis by piezo-transducers". Earthquake engineering and structural dynamics, 32(12), 2003, Pages 1897-1916.

35. A. R. De Faria, "The effect of finite stiffness bonding on the sensing effectiveness of piezoelectric patches". Technical note, smart materials and structures, 12, 2003, Pages 5-8.

36. S. Bhalla and C. K. Soh, "High frequency piezoelectric signature for diagnosis of seismic/blast induced structural damage". NDT\&E international, 37(1), 2004, Pages 23-33.

37. S. Bhalla and C. K. Soh, "Structural health monitoring by piezoimpedance transducers". Journal of aerospace engineering, ASCE, $17(4)$.

38. R. K. Ing and M. Fink, "Time reversed Lamb waves". IEEE transactions on ultrasonics, ferroelectrics, and frequency control, Vol. 45, No. 4, 1998, Pages 1032-1043.

39. J. Berryman, L. Borcea, G. Papanicolaou and C. Tsogka, "Statistically stable ultrasonic imaging in random media". Journal of the acoustical society of America, Vol. 112, 2002, Pages1509-1522.

40. M. Fink, "Time reversal of ultrasonic fields-Part I: Basic principles'. IEEE transactions on ultrasonics, ferroelectrics and frequency control, Vol. 39, No.5, 1992, Pages 555-566.

41. C. S. wang, J. T. roseand F. K. chang, "A computerized time reversal method for structural health monitoring". Proceedings of the SPIE, Vol. 5046, 2003, Pages 48-58.

42. H. W. Park, H. Sohn, K. H. Law and C. R. Farrar, "Time reversal active sensing for health monitoring of a composite plate". Journal of sound and vibration, 2004.

43. S. Degala, P. Rizzo, K. Ramanathan, K. A. Harries, "Acoustic emission monitoring of CFRP reinforced concrete slabs". 
Construction and building materials, Vol. 23(5), 2009, Pages 20162026.

44. P. Shull, "Nondestructive evaluation: theory techniques and applications". Shull Peters J, editor. NY: M. Dekker; 2002.

45. M. Watanabe, M. Enokians T. Kishi, "Fracture behavior of ceramic coatings during thermal cycling evaluated by acoustic emission method using laser interferometers". Materials science and engineering A- structural materials and properties, microstructure and processing, 359(1-2), 2003,Pages 368-374.

46. M. Barbezat, A. J. Brunner, C. Huber and P. Flueler, "Integrated active fiber composite elements: Characterization for acoustic emission and acoustic ultrasonics". Journal of intelligent material sustems and structures, Vol. 18, 2007, Pages 515-525.

47. J. F. C. Markmiller and F. K. Chang, "Sensor network optimization for a passive sensing impact detection technique". Structural health monitoring, Vol. 9, 2010, Pages 25-39.

48. L. Liu and F. G. Yuan, "Active damage localization for plate-like structures using wireless sensors and a distributed algorithm". Smart materials and structures, Vol. 17, 2008, Pages 1-12.

49. P. F. Rizos, N. Aspragathos and A. D. Dimarogonas, "Identification of crack location and magnitude in a cantilever beam from the vibration modes". Journal of sound vibration 138 (3), 1990, Pages 381-388.

50. H. F. Lam and C. T. Ng, "A probabilistic method for the detection of obstructed cracks of beam type structures using spatial wavelet transformation”. Probabengmech 23 (2-3), 2008, Pages 237-245.

51. K.V. Yuen and H.F. Lam, "On the complexity of artificial neural networks for smart structures monitoring".EngStruct28 (7),2006, Pages 977-984.

52. H.F. Lam, K.V. Yuen and J.L. Beck, "Structural health monitoring via measured Ritz vectors utilizing artificial neural networks".Comput-Aided CivInfrastructEng21 (4) (2006), Pages $232-241$. 
53. H.F. Lam, J.M. Ko and C.W. Wong, "Localization of damaged structural connections based on experimental modal and sensitivity analysis".Journal of Sound Vibration210 (1), 1998, Pages 91-115.

54. T. Yin, H.F. Lam, H.M. Chow and H.P. Zhu, "Dynamic reduction" based structural damagedetection of transmission tower utilizing ambient vibration data”.EngStruct31 (9), 2009, Pages2009-2019.

55. P. Cawley and R.D. Adams, "The location of defects in structures from measurements of natural frequencies".J Strain Anal14 (2), 1979, Pages 49-57.

56. H.P. Chen and N. Bicanic, "Assessment of damage in continuum structures based on incomplete modal information".ComputStruct74 (5), 2000, Pages 559-570.

57. L.H. Yam, Y.Y. Li and W.O. Wong, "Sensitivity studies of parameters for damage detection of plate-like structures using static and dynamic approaches".EngStruct24 (11) (2002), Pages $1465-1475$.

58. M. Tanaka and H. Tsuboi, "Finite element model of naturalcrack in eddy current testing problem".IEEE Trans Magn37 (5), 2001, Pages $3125-3128$.

59. H. F. Lam and T. Yin, "Statistical detection of multiple cracks on thin plates utilizing dynamics response". Engineering structures, Vol. 32, issue 10, 2010, Pages 3145-3152.

60. C. Biemans, W.J. Staszewski, C. Boller and G.R. Tomlinson, "Crack detection in metallic structures using piezoceramic sensors".Key Eng Mater167-168, 1999, Pages 112-121.

61. P.S. Tua, S.T. Quek and Q. Wang, "Detection of cracks in cylindrical pipes and platesusing piezo-actuated Lamb waves'.Smart Mater Struct,14, 2005, Pages 1325-1342.

62. J.-B. Ihn and F.-K. Chang, "Detection and monitoring of hidden fatigue crack growth using a built-in piezoelectric sensor/actuator network: I".Diagnost, Smart Mater Struct,13, 2004, Pages 609-620.

63. Y. Lu, L. Ye, Z. Su and C. Yang, "Quantitative assessment of through-thickness crack size based on Lamb wave scattering in aluminum plates". NDT \& E international, Vol. 41, issue 1, 2008, Pages 59-68. 
64. R.J. Ditchburn, S.K. Burke and C.M. Scala, "NDT of welds: state of the art”.NDT\&E Int.29, 1996, Pages 111-117.

65. J. Krautkrämer and H. Krautkrämer, "Ultrasonic Testing of Materials (3rded.)”. Springer-Verlag, Berlin 1983.

66. S. Dixon, C. Edwards and S. B. Palmer, "A laser-EMAT system for ultrasonic weld inspection". Ultrasonics, Vol. 37, Issue 4, 1999, Pages 273-281.

67. S. Grondel, J. Assaad, C. Delebarre, P. Blanquet and E. Moulin, "The Propagation of Lamb Waves in multilayered plates: phased velocity measurement," Meas. Sci. Technl. 10, 1999, Pages 348-353.

68. I. A.Viktorov, "Rayleigh and Lamb Waves," New York: Plenum, 1967.

69. A. H.Nayfeh and D. E.Chimenti, "Free waves propagation in plates of general anisotropic media," J. Appl. Mech. 56, 1989, Pages 881887. 